\title{
Texture Representation for Low-resolution Palmprint Recognition
}
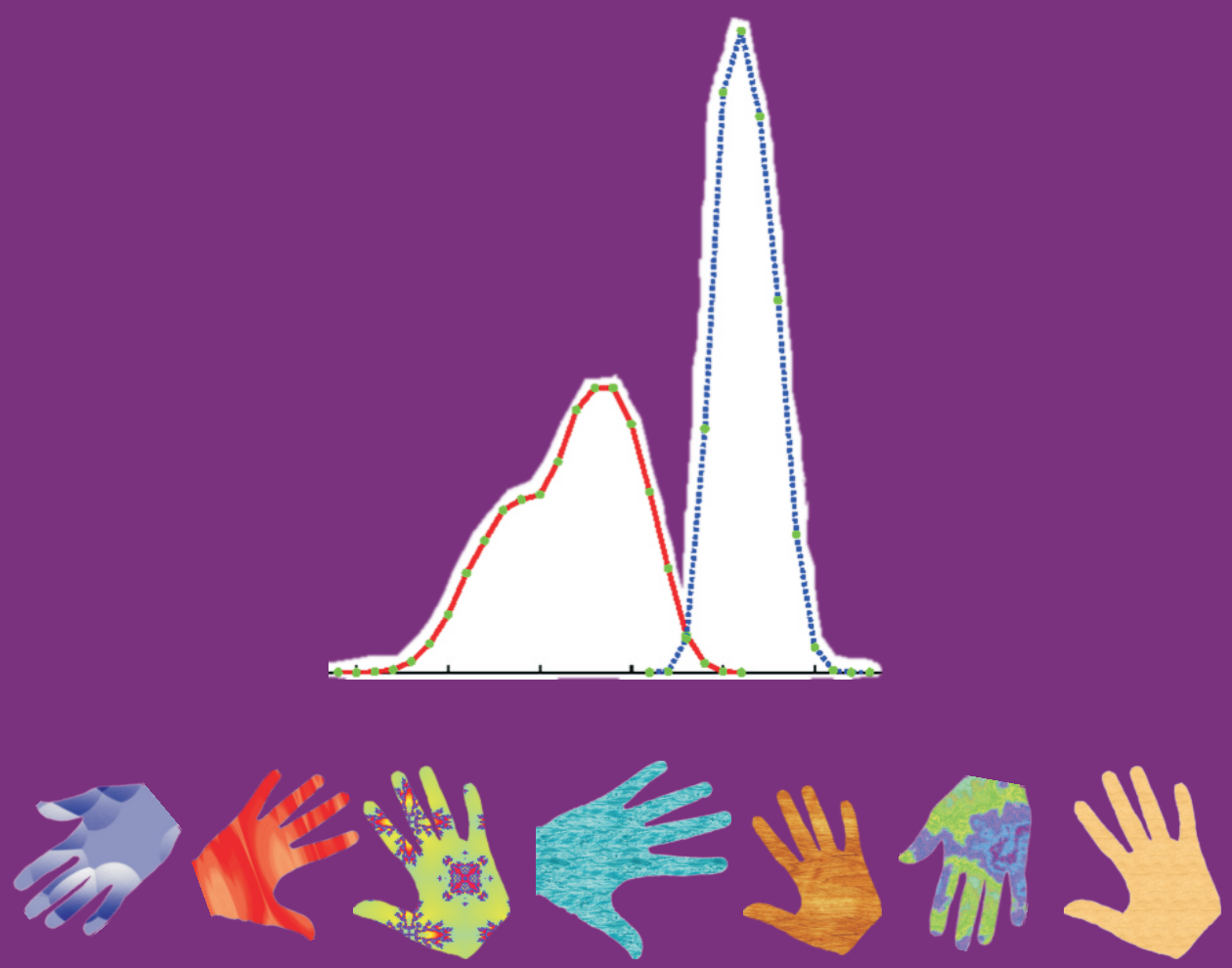

Meiru Mu 


\section{TEXTURE REPRESENTATION FOR LOW-RESOLUTION PALMPRINT RECOGNITION}

Meiru Mu 
De promotiecommissie:

voorzitter en secretaris:

Prof.dr.ir. A.J. Mouthaan University of Twente promotor:

Prof.dr.ir. C.H. Slump University of Twente

Prof.dr.ir. R.N.J. Veldhuis University of Twente assistent promotor:

Dr.ir. L.J. Spreeuwers University of Twente leden:

Prof.dr. P.H. Hartel University of Twente

prof.dr.ir. M.G. Vosselman University of Twente

Prof.dr.ir. M.J.T. Reinders Delft University of Technology

Prof.dr. J. Bigun Halmstad University

CTIT Ph.D.-thesis Series No. 13-255

Centre for Telematics and Information Technology

University of Twente

P.O. Box 217, NL - 7500 AE Enschede

ISSN 1381-3617

ISBN 978-90-365-0007-4

DOI: $10.3990 . / 1.9789036500074$

The work described in this thesis has been carried out

at the Institute of Information Science, Beijing Jiaotong University, China and at the Signals \& Systems group, University of Twente, The Netherlands.

(C)Meiru Mu, Enschede, 2013

No part of this publication may be reproduced by print, photocopy or any other means without the permission of the copyright owner.

Cover designed by: Meiru Mu

Printed by: Gildeprint Drukkerijen - Enschede 


\title{
TEXTURE REPRESENTATION FOR LOW-RESOLUTION PALMPRINT RECOGNITION
}

\author{
PROEFSCHRIFT
}

\author{
ter verkrijging van \\ de graad van doctor aan de Universiteit Twente, \\ op gezag van de rector magnificus, \\ prof. dr. H. Brinksma, \\ volgens besluit van het College voor Promoties \\ in het openbaar te verdedigen \\ op vrijdag 5 Juli 2013 om 14.45 uur
}

door

\section{Meiru Mu}

geboren op 27 Oktober 1984

te Shanxi, China 
Dit proefschrift is goedgekeurd door:

De promotor:

Prof.dr.ir. C.H. Slump

Prof.dr.ir. R.N.J. Veldhuis

De assistent promotor:

Dr.ir. L.J. Spreeuwers 


\section{Contents}

Summary v v

$\begin{array}{ll}\text { Samenvatting ix } & \text { ix }\end{array}$

1 Introduction 1

1.1 Biometric systems . . . . . . . . . . . . . . . 1

1.1.1 System modules . . . . . . . . . . . . . . 1

1.1.2 Risk and template protection . . . . . . . . . . 3

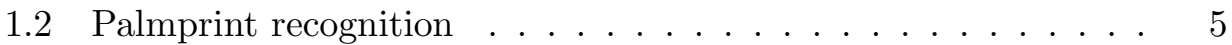

1.2.1 Palmprint features . . . . . . . . . . . . 6

1.2.2 Online palmprint recognition . . . . . . . . 8

1.3 Research content . . . . . . . . . . . . . . . . 9

1.3.1 The selected target applications . . . . . . . . . . 10

1.3.2 The selected template protection scheme . . . . . . . . 11

1.3.3 Research objectives . . . . . . . . . . . . . . 12

1.4 Overview of the thesis . . . . . . . . . . . . 13

1.4.1 Main contributions . . . . . . . . . . . . . 13

1.4.2 Chapters overview . . . . . . . . . . . . . . 14

1.4.3 Biometric data sets . . . . . . . . . . . . . 18

2 Region covariance matrices $\quad 21$

2.1 Chapter introduction . . . . . . . . . . . . . . . . 21

2.2 Region covariance matrices as representation . . . . . . . . . 23

2.2 .1 Abstract. . . . . . . . . . . . . 23

2.2 .2 Introduction . . . . . . . . . . . . . 23

2.2 .3 Related work . . . . . . . . . . . . . . 26

2.2.4 Log_GMP based RCMs as palmprint descriptors . . . . 28 
2.2.5 Experimental results . . . . . . . . . . . . . . . 34

2.2 .6 Conclusion and future work . . . . . . . . . . 37

2.3 Chapter conclusion . . . . . . . . . . . . . . . . 38

3 Mean and standard deviation of Gaussian 39

3.1 Chapter introduction . . . . . . . . . . . . . . . . 39

3.2 Mean and standard deviation as representation . . . . . . . 41

3.2 .1 Abstract . . . . . . . . . . . . . . . . . 41

3.2 .2 Introduction . . . . . . . . . . . . . . . 41

3.2.3 Palmprint recognition based on Gabor filtered images . 45

3.2 .4 Experimental results . . . . . . . . . . . . . . 53

3.2 .5 Conclusion . . . . . . . . . . . . . . 61

3.3 Chapter conclusion . . . . . . . . . . . . . . . . . 62

4 Local binary pattern histogram $\quad 63$

4.1 Chapter introduction . . . . . . . . . . . . . . . . 63

4.2 Shift and gray scale invariant representation on CDFB and LBP 65

4.2 .1 Abstract . . . . . . . . . . . . . . 65

4.2 .2 Introduction . . . . . . . . . . . . . . 65

4.2.3 Feature extraction using complex directional wavelet and local binary pattern . . . . . . . . . . . . . 69

4.2.4 Experimental results . . . . . . . . . . . . . . 73

4.2.5 Conclusion and future work . . . . . . . . . . . 87

4.3 Chapter conclusion . . . . . . . . . . . . . . . . 88

5 Fourier spectrum of PalmCode $\quad 89$

5.1 Chapter introduction . . . . . . . . . . . . . . . . . 89

5.2 Fourier spectrum of PalmCode as representation . . . . . . . 91

5.2 .1 Abstract . . . . . . . . . . . . . . . . 91

5.2 .2 Introduction . . . . . . . . . . . . . . . . 99

5.2 .3 Review of PalmCode . . . . . . . . . . . . . . 94

5.2 .4 Fourier spectrum of PalmCode . . . . . . . . . . . 95

5.2.5 Spectral feature reduction by $(2 \mathrm{D})^{2} \mathrm{PCA} \ldots \ldots \ldots 98$

5.2 .6 Experimental results . . . . . . . . . . . . . . 101

5.2 .7 Conclusion and future work . . . . . . . . . . . . 109

5.3 Chapter conclusion . . . . . . . . . . . . . . . . . 110

6 Binary representation on one-bit quantization 111

6.1 Chapter introduction . . . . . . . . . . . . . . . . . 111

6.2 Binary features on Gabor filtering and one-bit quantization . . 113

6.2.1 Abstract . . . . . . . . . . . . . . 113 
6.2 .2 Introduction . . . . . . . . . . . . . . . 113

6.2.3 Gabor statistical feature extraction . . . . . . . . . 115

6.2.4 One-bit quantization and reliable bits selection . . . . . 117

6.2.5 Experimental results . . . . . . . . . . . . . . . . 119

6.2.6 Conclusion and discussion . . . . . . . . . . . . . 121

6.3 Chapter conclusion . . . . . . . . . . . . . . . . . . 122

7 Binary representation on multi-bit quantization $\quad \mathbf{1 2 5}$

7.1 Chapter introduction . . . . . . . . . . . . . 125

7.2 Binary features on Gabor filtering and multi-bit quantization . 127

7.2 .1 Abstract . . . . . . . . . . . . . 127

7.2 .2 Introduction . . . . . . . . . . . . . 127

7.2.3 Brief review of LogGM feature . . . . . . . . . . 130

7.2.4 Binary LogGM_DROBA feature extraction . . . . . . . 131

7.2 .5 Experimental results . . . . . . . . . . . . . . . 133

7.2 .6 Conclusion and outlook . . . . . . . . . . . . 140

7.3 Chapter conclusion . . . . . . . . . . . . . . . . . . 141

8 Conclusions and Future Work 143

8.1 Objectives and contributions . . . . . . . . . . 143

8.2 Discussion of achievements . . . . . . . . . . . . 144

8.3 Future work . . . . . . . . . . . . . . . . 148

$\begin{array}{lr}\text { References } & 149\end{array}$

$\begin{array}{ll}\text { Acknowledgements } & 161\end{array}$

$\begin{array}{ll}\text { Curriculum vitae } & 163\end{array}$ 


\section{Summary}

Person recognition plays an important role in our society and world. This can be observed in varieties of application scenarios such as access control, data management, national ID and forensics. The typical approaches for linking an individual to his/her identity are based on the personal possessions (what you have) or knowledge (what you know), which have the disadvantages of constantly being forgotten, lost or stolen. In the last decades, person identification based on "who you are" has been intensively developed. This is commonly referred to as Biometrics. In this field, the link between an individual and his/her identity is automatically and uniquely established by a human's intrinsic physiological or behavioral trait, such as face, iris, fingerprint, palmprint, finger vein pattern, voice, signature, gait, and so on. For a biometric system, the recognition performance basically depends on the quality of the captured image, the discriminative ability of the extracted features and the classification performance of the employed classifier. It is a challenge to construct a highly discriminative representation from the captured biometrical images, due to their intra-class variations and inter-class similarities. Meanwhile, the widespread use of biometric systems creates security and privacy risks, which have been concerned with increasing attention recently. To mitigate those risks, template-protection technology has been developed as a solution to safeguarding the stored biometric templates. For its successful implementation, the biometrical representation is generally required to be quantized into bits, which are expected to be as discriminative and reliable as possible. This is challenging since the biometric data is highly noisy.

Our research focuses on palmprint recognition, since the palmprint carries rich discriminative features, including principle lines, wrinkles, ridge, valleys, and minutiae. For a specific application, the feature selection is determined by the image quality. For instance, in applications of forensic and law enforcement, the captured images are generally high-resolution (more than $500 \mathrm{dpi}$ ). 
The ridge patterns, minutiae points and suchlike, which are more common in fingerprint images, can be deployed for discriminative feature extraction. On the other hand, in real-time person recognition systems, the palmprint images are usually low-resolution (less than $100 \mathrm{dpi}$ ). The distinctive features are principle lines and wrinkles. This thesis investigates how to extract features from low-resolution palmprint images for online (real-time) person recognition. The images are typically captured by a scanner or a CCD sensor. The major palmprint characteristics, which we can detect and process for representation, are lines and wrinkles. In this thesis, these low-resolution palmprint images are treated as texture images. Accordingly, texture analysis technologies are mainly investigated for palmprint representation.

In the first half of this thesis, finding a suitable mechanism to extract realvalued features from low-resolution palmprint is studied. The research subject is how to extract the invariant features. Here, "invariant" means "being robust to the within-class image variations such as translation, rotation, and illumination changes". In general, those image variations are caused by sensor noises, environment condition changes, and user's gesture varieties. The palmprint recognition system is considered to work in identification mode. Identification rate and processing time are the major performance indicators. Firstly, the approach of region covariance matrices (RCM) as feature descriptor is developed for palmprint representation, due to its advantages of low dimensionality, being scale and illumination independence. For constructing the discriminative RCM, a suitable feature mapping vector is required. The novelty of this work is that we resort to the Gabor magnitude and phase information, and especially we transform the Gabor magnitude coefficients into log-scale for building the feature mapping vector. Accordingly, the generated RCM representation turns out to be of high discriminative ability. Secondly, a novel representation constructed by groups of two simple statistics (mean and standard deviation) is proposed, which is based on the findings that Gabor magnitude coefficient matrices approximate the lognormal distributions. The statistical features are extracted from some partitioned sub-blocks so as to be robust against the slight image deformation. Thirdly, the complex directional filter bank (CDFB) transform and the local binary pattern (LBP) operator are investigated for palmprint representation. The CDFB can generate an energy shiftable and scalable multi-resolution decomposition, and the LBP is a grayscale invariant operator. The proposed representation is based on combining the CDFB transform and the LBP coding. Compared with the other multiscale and multidirectional transforms, CDFB outperforms in terms of higher identification rate, less storage requirement and lower computational complexity when it is combined with LBP coding. Finally, the coding-based methods 
reported in many publications for palmprint verification are further studied. The extracted code features are highly discriminative, but they are sensitive to the image translation. To offset it, some code-plane alignment operations are commonly implemented for similarity measurement. This process suffers from low matching speed when the system works in identification mode. Accordingly, the Fourier spectrum of palm code is proposed as feature descriptor, which is based on the translation invariance property of discrete Fourier transform (DFT) and two-dimensional horizontal and vertical principle component analysis projection $\left((2 \mathrm{D})^{2} \mathrm{PCA}\right)$.

In the second part of the thesis, the focus shifts towards constructing reliable binary palmprint representations so that the palmprint recognition system can be combined with template protection techniques for higher security. The Helper Data Scheme (HDS) is considered as the subject for evaluating the performance of our proposed binary representation algorithms for palmprint template protection. The performance indicators include verification accuracy, bit error rate of extracted binary strings, and the length of secret key, which is combined with the extracted bits. For extracting binary features, the basic strategy is quantizing the real-valued palmprint representation into bits. With regards to the real-valued palmprint representation for quantization, the mean and standard deviation based features are chosen as the major quantization object, which we propose in the first part of the thesis. By using these features, the one-bit and multi-bit equal-probability-interval quantization methods are investigated in sequence, together with their corresponding reliable bit selection approaches. Compared with the classical coding-based methods for unprotected system, our proposed binary representations can achieve much lower bit error rate (BER) for genuine matching, which is essential for building a successful template protection system. 


\section{Samenvatting}

Persoonsherkenning speelt een belangrijke rol in onze samenleving en onze wereld. Dat blijkt uit de vele toepassingsgebieden zoals toegangscontrole, data management, identiteitskaarten en forensische toepassingen. De gangbare manieren om een individu aan zijn of haar identiteit te koppelen zijn gebaseerd op persoonlijke bezittingen (wat je hebt) of kennis (wat je weet). Deze hebben het nadeel dat ze regelmatig worden vergeten, verloren of gestolen. Gedurende de afgelopen tientallen jaren is persoonsidentificatie op basis van "wie je bent" intensief ontwikkeld. Dit wordt in het algemeen aangeduid met biometrie. In de biometrie gebeurt de koppeling van een individu aan zijn of haar identiteit automatisch en op unieke wijze via een intrinsiek fysiologisch of gedragskenmerk. Voorbeelden hiervan zijn gezicht, iris, vingerafdruk, handpalmafdruk, vingeraderpatroon, stem, handtekening, manier van lopen enzovoorts. De betrouwbaarheid van de herkenning door een biometrisch systeem hangt in principe af van de kwaliteit van het opgenomen beeld, het onderscheidingsvermogen van de getraheerde kenmerken en de prestaties van de gebruikte classificator. Het is een uitdaging om een representatie uit te biometrische beelden te realiseren die in een hoog onderscheidingsvermogen resulteert, want er zijn vaak overeenkomsten tussen verschillende klassen en variaties binnen een klasse. Intussen veroorzaakt het toenemende gebruik van biometrische systemen veiligheids- en privacyrisico's welke ook steeds meer aandacht krijgen. Om deze risico's te verkleinen is de template-protectie-techniek ontwikkeld met als doel de opgeslagen biometrische templates te beveiligen. Voor de toepassing van deze techniek is het meestal nodig om de biometrische representatie te quantiseren in bits die zo onderscheidend en betrouwbaar mogelijk moeten zijn. Dit is een uitdaging, want biometrische data bevat veel ruis.

Ons onderzoek concentreert zich op handpalmafdrukherkenning, want de handpalmafdruk heeft vele onderscheidende kenmerken, waaronder de principale lijnen, plooien, richels, dalen, en minutiae. Voor een specifieke toepassing 
wordt de selectie van kenmerken bepaald door de beeldkwalitiet. Bijvoorbeeld voor forensische toepassingen hebben de beelden meestal een hoge resolutie (meer dan 500 dpi). De richelpatronen, munitiaepunten enzovoorts, die meer gebruikelijk zijn in vingerafdrukherkenning, kunnen dan gebruikt worden als discriminatieve kenmerken. Aan de andere kant, in real-time persoonsherkenningssystemen hebben de beelden van de handpalmafdruk gewoonlijk een zeer lage resolutie (minder dan 100 dpi). De discriminatieve kenmerken zijn dan de principale lijnen en de plooien. Dit proefschrift onderzoekt hoe kenmerken kunnen worden getraheerd uit lage-resolutiebeelden van hadpalmafdrukken voor online (real-time) persoonsherkenning. Deze beelden worden in het algemeen opgenomen m.b.v. een scanner of een CCD-sensor. De belangrijkste kenmerken van de handpalmafdruk die we dan kunnen detecteren en analyseren zijn lijnen en plooien. In dit proefschrift worden de lage-resolutiebeelden van de handpalm gezien als textuurbeelden. Daarom worden voornamelijk textuuranalysetechnieken onderzocht voor het realiseren van een representatie van de handpalm.

In de eerste helft van dit proefschrift wordt het vinden van een geschikte aanpak voor extractie van reelwaardige kenmerken uit lage-resolutiebeelden van de handpalm onderzocht. Het doel van het onderzoek is het vinden van invariante kenmerken. "Invariant" betekent hier robuust tegen variaties binnen een beeldklasse, zoals translatie, rotatie en variatie in belichting. Deze variaties worden in het algemeen veroorzaakt door ruis, omgevingsinvloeden en variaties in gebaren van de gebruiker. We veronderstellen dat het handpalmherkenningssysteem voor identificatie wordt gebruikt. De identificatiescore en de snelheid zijn de belangrijkste prestatie-indicatoren. Eerst wordt de methode gebaseerd op regio-covariantiematrices (RCM) als kenmerkbeschrijving ontwikkeld voor handpalmrepresentatie vanwege zijn lage dimensionaliteit en het feit dat hij schaal- en belichtingsonafhankelijk is. Voor de constructie van een discriminatie RCM is een geschikte kenmerktransformatie nodig. De innovatie van dit werk is dat we de Gabor magnitude en fase gebruiken en in het bijzonder dat we die naar een logaritmische schaal transformeren om de kernmerkvector te realiseren. De resulterende RCM representatie blijkt een groot onderscheidend vermogen te hebben. Ten tweede wordt een representatie bestaande uit twee eenvoudige statististieken (gemiddelde en standaard deviatie) voorgesteld, welke is gebaseerd op de waarneming dat Gabormagnitude-cofficint-matrices de lognormaal verdeling benaderen. De statistische kenmerken worden gextraheerd uit gepartitioneerde subblokken om robuust te zijn tegen kleine beelddeformaties. Ten derde worden de Complex Directionele Filter Bank (CDFB) transformatie en de Local Binary Patterns (LBP) operator onderzocht voor de representatie van de handpalmafdruk. De CDFB- 
transformatie kan een multi-resolutie decompositie realiseren die getransleerd kan worden en schaalbaar is. De LBP-operator is een grijsschaal-invariante transformatie. De voorgestelde representatie is gebaseerd op een combinatie van de CDFB-transformatie en LBP-codering. Vergeleken met andere multischaal en multi-directionele transformaties geeft CDFB hogere identificatiescores en heeft minder opslagruimte nodig en heeft een lagere complexiteit als hij wordt gecombineerd met LBP-codering. Tenslotte worden ook andere codegebaseerde methoden uit verschillende publicaties verder bestudeerd. De gextraheerde code-kenmerken zijn zeer discriminatief, maar zijn wel gevoelig voor beeldtranslaties. Daarom worden beelden meestal uitgelijnd als ze worden vergeleken. Dit proces resulteert wel in een lage vergelijkingssnelheid als het systeem voor identificatie wordt gebruikt. Daarom wordt het Fourier-spectrum van de palm code voorgesteld als kenmerkbeschrijving, welke is gebaseerd op de translatie-invariante eigenschap van de Discrete Fourier Transformation (DFT) en de tweedimensionale horizontale en verticale principale component analyse projectie $\left((2 \mathrm{D})^{2} \mathrm{PCA}\right)$.

Het tweede deel van het proefschrift gaat over het construeren van betrouwbare binaire handpalmrepresentaties, zodat het handpalmafdrukherkenningssysteem kan worden gecombineerd met templateprotectietechnieken voor een grotere veiligheid. Het Helper Data Schema (HDS) wordt gebruikt voor de prestatieevaluatie van de binaire representaties voor templateprotectie die we hebben voorgesteld. De prestatie-indicatoren omvatten verificatienauwkeurigheid, bit error rate (BER) van de gextraheerde binaire reeksen en de lengte van de geheime sleutel die met de gextraheerde bits wordt gecombineerd. Om binaire kenmerken te extraheren wordt in principe de reelwaardige handpalmrepresentatie gequantiseerd in bits. Uit de reelwaardige handpalmrepresentatie worden de kenmerken gebaseerd op het gemiddelde en de standaard deviatie gekozen voor quantisatie. Op basis van deze kenmerken worden 1-bit en multi-bit quantisatiemethoden onderzocht met de bijbehorende betrouwbarebit-selectie technieken. Vergeleken met de klassieke code-gebaseerde methoden zonder templateprotectie, heeft onze methode een veel lagere bit error rate (BER) voor vergelijking van twee handpalmafdrukken van dezelfde persoon, wat essentieel is voor een succesvol templateprotectiesysteem. 


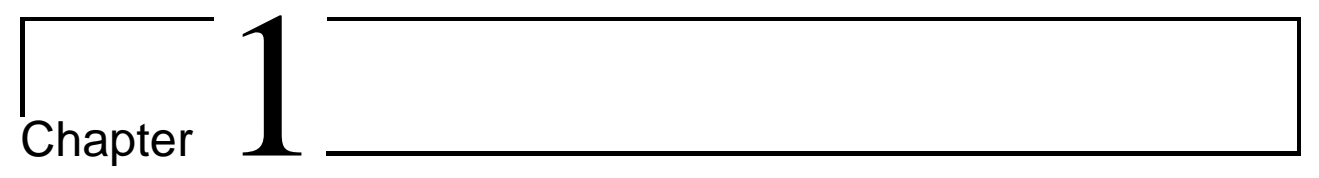

\section{Introduction}

\subsection{Biometric systems}

Biometrics refers to the technologies which are for an individual's identity establishment by measuring his/her physical characteristics or behavioral traits.

As our information society is getting more and more intelligent, the requirement for automatic individual identity establishment is being widely spread and enhanced. The application areas involve access control, forensics, data management, national ID, passport control, and so on. The traditional means of automatic person recognition are based on individual's knowledge (e. g. passwords) or possessions (e. $g$. ID cards). As the social communication scale enlarges, those means are suffering from knowledge being forgotten or shared, and possessions being stolen or manipulated. In contrast, biometrics offers a more efficient solution by enhancing user convenience and reducing system security risks, owing to its natural way of linking an individual's identity with his/her unique biological characteristics.

\subsubsection{System modules}

In reality, the biometric recognition applications appear to be of different characteristics. For instance, the user is cooperative or not, the system op- 


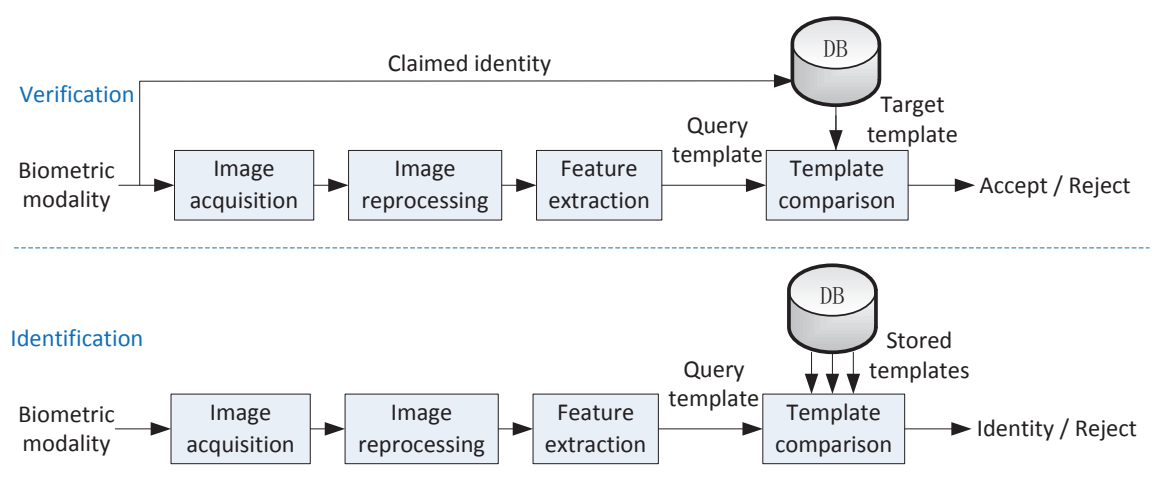

Fig. 1.1: The block diagrams of biometric system on identification and verification operation modes respectively.

erates in a controlled environment or not, and the biometric data is used by single application or multiple applications. However, the systems typically operate in three modes: enrollment, identification, and verification. The enrollment stage is for collecting users' information and feature templates (called reference templates) from their biometrical modalities. The identification or verification (recognition is a general term for both) stage is for extracting the feature template from a test image, and then implementing comparison between the test template and the reference template. Figure 1.1 illustrates the system's structure diagrams for identification and verification operation modes respectively. Three basic processing modules are usually involved in both of operation modes, including image acquisition, image reprocessing, and feature extraction. Image acquisition block is depended on the processed biometrical modality and sensor device. Image reprocessing block generally involves object segmentation, image enhancement, registration, region of interest cropping, image quality assessment, and so on. Feature extraction block generates a compact collection of biometric features, which is called biometric template in general. In the enrollment mode, the obtained feature templates are stored in a database.

About the template comparison, identification and verification blocks work in different ways, as can be seen in Fig. 1.1. In identification mode, the system output is an identity, which is established by comparing the query template with all the templates stored in database (one-to-many comparison). The identity established by system is either correct or wrong. In general, the identification performance indicators are used as following:

- Identification rate (IR), which is the rate of correctly obtaining the identity 
of a query template.

- Feature template size.

- Average processing time of feature extraction and matching.

The feature template size and the average processing time are for evaluating the memory and computational requirements of algorithm. In verification mode, the user's identity is verified by comparing his/her feature template with that of the claimed identity (one-to-one comparison). In general, the verify decision is made on the comparison between the matching score and the threshold. If the matching score is larger than the threshold, a match will be returned and the query identity will be accepted. Otherwise, a nonmatch will be returned and the query identify will be rejected. A comparison between biometric samples of the same individual is usually referred to as genuine comparison, and a comparison between biometric samples of different individuals is referred to as impostor comparison. A non-match returned from the genuine comparison results in a false rejection, and a match returned from the imposter comparison results in a false acceptance. To evaluate the system performance, the generally used indicators are as following:

- False Acceptance Rate (FAR), which is the probability of a match decision returned from an impostor comparison.

- False Rejection Rate (FRR), which is the probability of a non-match decision returned from a genuine comparison.

- Equal Error Rate (EER), which is determined by a threshold where the resulted FAR is equal to corresponding FRR.

- Genuine Acceptance Rate (GAR), which is the probability of a match decision returned from a genuine comparison. GAR=1-FRR.

- Receiver Operating Characteristics (ROC) curve, which plots the FAR against the FRR by adapting the threshold. It presents the performance of a biometric system by visualizing the character of the trade-off between the FAR and the FRR. Sometime, this curve is also referred to as Detection Error Trade-off (DET) curve, or plots the GAR against the FRR.

\subsubsection{Risk and template protection}

Compared with the traditional person recognition methods, Biometrics offers the great advantages. The covert collection and use of biometric data has been widely spreading, as the Biometric and social networking technologies emerge and develop. In consequence, it entails user privacy and system se- 
curity concerns. The compact bond between the digital representation and the physiological or behavioral body properties challenges privacy and security. A security risk is defined as a vulnerability of the system that facilitates an adversary to attack the system or increases the adversary's success rate of attacking the system. Privacy risks are related to vulnerabilities in which the adversary extracts valuable information about the individuals that use the biometric system. In general, there are several security and privacy threats:

(1) Identity fraud: where an adversary impersonates the genuine subject of the system by some spoofing mechanism or by stealing the stored reference template. For example, face images can be easily acquired on a distance. Fingerprints are left unintentionally on surfaces of objects that we touch in everyday life. Both modalities are widely utilized in crime investigation. The systems based on them can be easily circumvented with fake artifacts.

(2) Limited-renewability: unlike passwords, biometric data implies the limited capability to be renewed. Once lost, lost forever.

(3) Leaking personal information: where it is known that biometric data is irrevocable or unchangeable, some of them may contain personal information such as health condition or ethnicity. To some extent biometric data should be classified as sensitive personal data. Loss or mishandling of such data might generate grave privacy concerns.

(4) Cross-matching: in case an individual is enrolled in several databases of different applications, it is possible for an attacker to track his/her behavior by linking reference templates across databases.

Mitigating the risks mentioned above is essential to obtain the acceptance from the subjects of the biometric systems and therefore to facilitate the successful implement on a large-scale. Currently, there have been some guidelines or policies to address this issue of biometric information protection. According to ISO guidelines [1], for stored biometric data the following requirements are included:

(1) Data minimization, referring to collecting the most necessary biometric data. For example, by storing the extracted feature templates instead of their corresponding biometric image samples, it can mitigate the risk of unauthorized use.

(2) Confidentiality, ensuring that only authorized persons can get access to the stored biometric data. It is a direct way to reduce the risk of unauthorized use and leaking the privacy information. 
(3) Integrity, guaranteeing that the stored biometric data cannot be modified without authorization.

(4) Irreversibility, implying that transforming the biometric sample into the feature template is a one-way function. It will be impossible or very difficult for an adversary to retrieve the original biometric sample from the stored template.

(5) Renewability, ensuring that different reference templates can be created from one biometric object in case one gets compromised.

(6) Unlinkability, emphatically pointing out that it is impossible or at least difficult to trace back to the same biometric object by linking those different templates derived from it.

To enforce those guideline there have been some countermeasures to safeguard the privacy and security. For example, we can store feature templates rather than the biometric samples. The privacy information can be stored on personal smart card or token rather than a centralized database. For integrity and confidentiality, the classical encryption techniques can be adopted during the process of data storage and transmission. For irreversibility, renewability and unlinkability, the template protection technique has been considered to be efficient and received significant attention from the research community. Currently, there have been different template-protection schemes developed and implemented, which can be found in [2] and [3].

\subsection{Palmprint recognition}

Palmprint, a kind of human physiological trait, has considerable potential for person recognition. It shares most of the discriminative features with fingerprints, and in addition, possesses a much larger skin area and other discriminative features such as principle lines and creases. Moreover, it is promising to combine palmprints with other hand-based biometrical modalities, such as hand shape, knuckle and palm vein, for achieving a recognition system of higher recognition accuracy and advanced user-friendliness.

A typical palmprint recognition system involves four modules, including palmprint image acquisition, image reprocessing, feature extraction, and template comparison, which are the same as what we present in Section 1.1.1. From the view point of palmprint acquisition, the palmprint recognition system can 


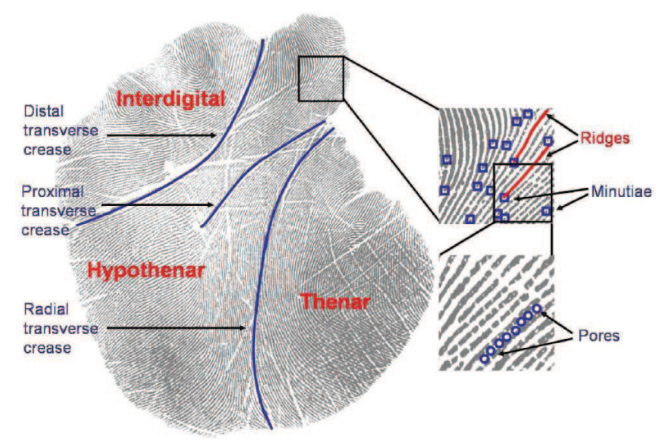

(a)

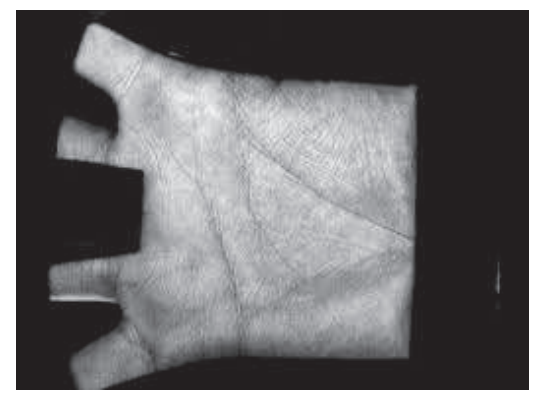

(b)

Fig. 1.2: Typical palmprint images in different resolution. (a) High resolution [5]; (b) Low resolution [6].

be either offline or online. The offline systems usually work in forensic applications, where palmprint recognition has a significant role since around $30 \%$ percent of the latents recovered from crime scenes are from palms [4]. The online systems are for civil and commercial applications such as access control. The image quality differs significantly for offline and online system. The palmprint images collected from criminal scenes are usually partial and can be digitalized into be high-resolution. Whereas the online palmprint images can be captured from the whole palm but the resolution is generally rather low so as to facilitate real-time processing.

\subsubsection{Palmprint features}

Figure 1.2 shows two typical palmprint images in different resolution. In general, there are two basic features in a palmprint: creases and ridges. Both of them are firmly attached to the dermis, and are immutable for the whole life. However, in the palmprint images of different qualities, creases and ridges possess their unique characteristics and therefore play different roles in varieties of applications, which are summarized in Table 1.1.

The ridges of palm are unique for an individual, just like those in fingerprint. They come into being during the three-to-four months of the fetal stage and are fixed in the adolescence stage [7]. Refer to [4], ridges can be further divided into ridge pattern, minutia points, ridge contours and pores. Ridge pattern and minutia points can be extracted from palmprint or fingerprint 
Table 1.1: Palmprint features.

\begin{tabular}{|c|c|c|}
\hline \multicolumn{2}{|r|}{ Type } & Characteristics \\
\hline Creases & $\begin{array}{l}\text { Principal lines } \\
\text { Wrinkles }\end{array}$ & $\begin{array}{l}\text { - } 100 \text { dpi } \\
\text { - Online low-resolution person recognition }\end{array}$ \\
\hline \multirow[t]{2}{*}{ Ridges } & $\begin{array}{l}\text { Ridge pattern } \\
\text { Minutia points }\end{array}$ & \multirow{2}{*}{$\begin{array}{l}\text { - } 500 \text { dpi for ridge patterns and minutia } \\
\text { - } 1000 \text { dpi for pores and ridge contours } \\
\text { - Latent palmprint recognition } \\
\text { - Forensics and law enforcement } \\
\end{array}$} \\
\hline & $\begin{array}{l}\text { Ridge contours } \\
\text { Pores }\end{array}$ & \\
\hline \multicolumn{2}{|c|}{ Multispectral features } & $\begin{array}{l}\text { - Infrared spectral imaging } \\
\text { - Multispectral data fusion for improving recognition } \\
\text { accuracy } \\
\text { - Palm vein information fusion for improving the ca- } \\
\text { pability of spoof detection }\end{array}$ \\
\hline \multicolumn{2}{|c|}{ 3D structural information } & $\begin{array}{l}\text { - } 3 \mathrm{D} \text { imaging } \\
\text { - More robust against fake palmprint attack } \\
-2 \mathrm{D} \text { and } 3 \mathrm{D} \text { information fusion for a high accuracy } \\
\text { and robust palmprint recognition system }\end{array}$ \\
\hline
\end{tabular}

with 500 or less dpi, while ridge contours and pores are from images with resolution of higher than 1000 dpi. Ridges features play an important role in the latent palmprint recognition, which has shown great potential in forensics and law enforcement. Figure 1.2(a) displays a typical palmprint image in forensic applications, where $500 \mathrm{dpi}$ is the standard resolution and latent-tofull matching must be supported.

Unlike fingerprint, there are many lines in palmprint such as three principle lines and wrinkles, which are generally referred to as creases. These main lines and wrinkles are formed several months after conception, and the other winkles are usually considered as the consequences of both genetic effects and various postnatal factors. Those complex line features turn out to be discriminative for person recognition. Compared to ridges, creases can be captured in the online systems with images of low resolution (around $100 \mathrm{dpi}$ ). Accordingly, they are the dominant features of low-resolution palmprint images, as Figure 1.2(b) shows. The online low-resolution palmprint recognition system only supports full-to-full palmprint matching.

Recently, with advance in sensor techniques and computational power, some other technologies are developed for online palmprint recognition systems, including multispectral palmprint recognition and 3D palmprint recognition. As 


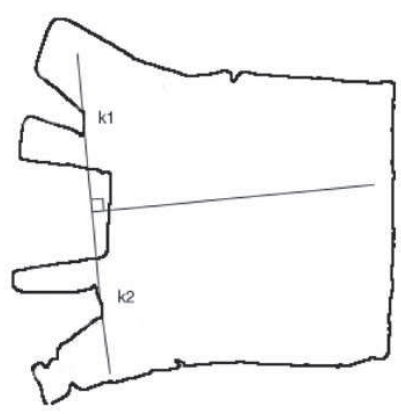

(a)

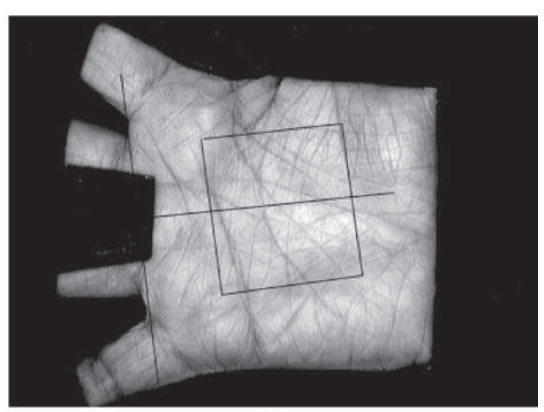

(b)

Fig. 1.3: An example of the palmprint ROI extraction process [12]. (a) Key points detection; (b) Coordinate system establishment and ROI cropping.

it shows in Table 1.1, these technologies are resorted to not only for improving recognition accuracy, but also for improving the capability of spoofing detection.

\subsubsection{Online palmprint recognition}

For the online palmprint recognition systems, there are varieties of image acquisition devices. According to the types of sensor, the main devices include digital scanner, CCD (Charge Coupled Device) based palmprint scanner, and web camera. Among them, the web camera is exploited for a touchless system, which usually suffer that the image quality is relatively low. At present, the images in public palmprint databases are basically from digital scanner and CCD based palmprint scanner.

After a palmprint image is captured, some procedures are required to be processed for cropping the region of interest (ROI). With a ROI of equal size extracted from each image, it will reduce the intra-class variations and further facilitate the feature extraction. However, it is a challenge to align the images for ROI cropping. In publications [8-11], the reader can find some different algorithms of ROI extraction, which usually depend on the image quality and the captured palm shape. The general idea involves three steps: (1) Detect key points, whose positions are relatively reliable; (2) Establish a uniform coordinate system based on the detected key points; (3) Crop a region of square shape under the established coordinate system. Figure 1.3 shows an example of ROI extraction. Two points around the roots of fingers are detected as 
the reference points for the coordinate system establishment. In general, the points are finally found out by a sequence of image processing procedures. However, for an individual, the detected positions of key points vary with samples, due to the image acquisition noise and the palm distortion. In consequence, there are unavoidable variations among the extracted ROI sub-images from intra-class palmprint images. In general, the image variations involve rotation, translation and illumination perturbation.

Palmprint feature extraction and matching are processed on the extracted ROI sub-images. Aiming to a palmprint recognition system of high accuracy, a variety of feature extraction and matching approaches have been proposed. Refer to [13], the proposed methods can be grouped into three classes: (1) Holistic-based approaches; (2) Local feature based approaches; (3) Hybrid approaches. By holistic-based approaches, the palmprint images are generally represented either in a spatial domain or a transform domain. The palmprint images can be treated as a vector, a 2D matrix, or a second order tensor and then processed by sorts of linear and nonlinear subspace analysis technologies. Moreover, the palmprints can be firstly transformed by an image transform technique, such as Fourier transform, discrete cosine transform (DCT), and Gabor transform, and then processed by subspace analysis technologies. Local feature based approaches mainly resort to detecting and matching the principle lines and wrinkles by some image processing technologies such as image segmentation and enhancement. Hybrid approaches involve the multiple palmprint representations fusion, or some hierarchical matching scheme.

\subsection{Research content}

This thesis focuses on the study of the palmprint feature extraction and matching algorithms. Since the palmprint recognition is a large-scale multiclass issue, the classifier design is out of our discussion. By the term "feature extraction and matching" it mainly refers to constructing the discriminative palmprint representations and their corresponding similarity measurements. The applied classification approach is the nearest neighbor. As it presents in Section 1.2.2, the image variations among intra-class ROI sub-images are unavoidable, including translation, rotation, and illumination perturbation, even though we have implemented the image alignment. Therefore, our research question is: 
How should we create the palmprint representations and their corresponding similarity measurements, which can distinguish the inter-class palmprints and meanwhile can be robust against the intra-class variations of translation, rotation, and illumination perturbation?

\subsubsection{The selected target applications}

To achieve the suitable representation, we need to consider what our target applications are, so that we can know what our specific research objectives are.

With regards to the target applications, two basic scenarios are considered:

I. Typical (unprotected) online palmprint recognition system;

II. Combine the palmprint recognition system with template protection schemes;

As we present in Section 1.2.1, the main palmprint features are creases for online palmprint recognition system. Varieties of line detection operators, such as Sobel and Canny operators, have been investigated for extracting palmprint features. Figure 1.4 shows a classical example of line detection method referring to [14]. These kinds of methods turn out to be a lack of robustness to intra-class image variations. Even though this drawback can be mitigated by rotating and shifting the reference templates and then matching multiple times for similarity measure, it is time consuming. For a large-scale identification system, the feature size and the execution speed are also the important factors. Accordingly, in this thesis, we resort to texture analysis technologies to extract discriminative palmprint features. On one hand, some region based texture statistical methods are investigated. On the other hand, the local pattern based texture operating approaches are studied.

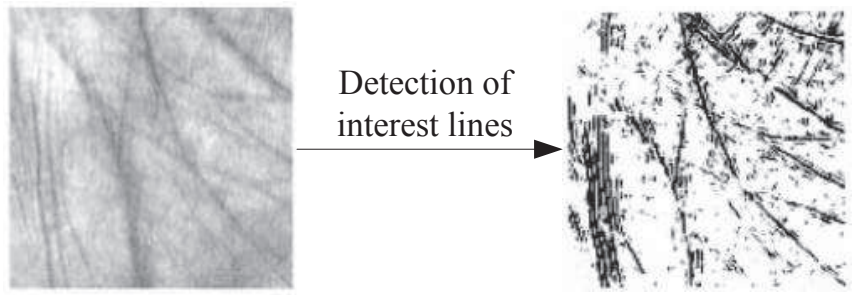

Fig. 1.4: An example of line detection method [14]. 


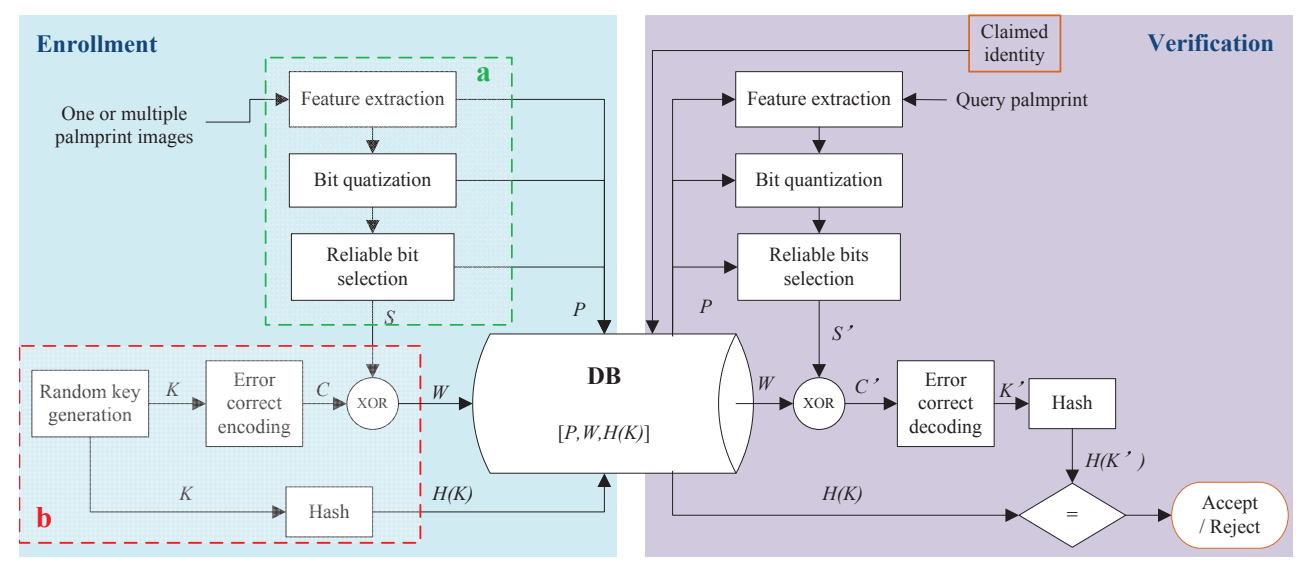

Fig. 1.5: An illustration of the palmprint verification system under the Helper Data Scheme. (a) Bit extraction and (b) Bit protection.

Regarding the palmprint template protection system, it is to cope with the system security and user privacy risks which have been a growing concern in biometrical applications. In this thesis, we resort to Helper Data Scheme (HDS) [2] as a solution of combing palmprint recognition system with template protection, which is targeted on the irreversibility, renewability and unlinkability properties.

\subsubsection{The selected template protection scheme}

In this section, we briefly present the targeted template protection scheme: Helper Data Scheme (HDS). Figure 1.5 illustrates the palmprint verification system under the HDS. It mainly consists of two parts: (a) Bit extraction and (b) Bit protection.

During the enrollment phase, multiple samples are usually acquired from one individual. By feature extraction module, a real-valued feature vector is extracted from each enrolled sample. After bit quantization and reliable bit selection, a single binary string is created from the multiple real-valued feature vectors. The bit quantization and selection could be user-specific. The first part (denoted by $P$ ) of helper data, including the related parameters for feature extraction and the user-specific information, needs to be stored as part of the protected template for use in the verification phase. For each user, a secret key $K$ is randomly generated and encoded into the codeword $C$. The 
second part of helper data is given by $W=C \oplus S$. The secret key $K$ is hashed into $H(K)$ by a one-way hash function, which is the third part of the helper data. All of these three parts $[P, W, H(K)]$ need to be stored as part of the protected template for use in the verification phase. The query palmprint is processed into a binary string $S^{\prime}$ under the help of data $P$. Then $C^{\prime}=S^{\prime} \oplus W$ is computed. By correcting the errors and decoding, $K^{\prime}$ is obtained. Finally, by comparing $H(K)$ with $H\left(K^{\prime}\right)$, the claimed identity will be rejected or accepted.

By analyzing the palmprint verification system under the HDS, we can conclude that the created palmprint representation (denoted by $S$ and $S^{\prime}$ in Figure 1.5) needs to be a binary string of fixed-length. For a verification system, it needs to be discriminative. In addition, the extracted bit strings from the intra-class images need to be reliable, since the error correcting capability of current error correct coding (ECC) techniques is limited. Furthermore, because the template similarity measurement is implemented in the Hash domain, the template alignment operation among templates is limited.

\subsubsection{Research objectives}

To summarize the contents in Sections 1.3.1 and 1.3.2, the research objectives can be considered as two parts:

A. Real-valued invariant feature representation for the unprotected palmprint recognition system;

B. Binary reliable feature representation for the palmprint template protection system.

Accordingly, the research question can be refined as followings:

\section{A. Given low-resolution palmprint images, how should we construct the feature representation for a recognition system with the follow- ing requirements?}

I. Since the image intra-class variations, such as translation, rotation and illumination perturbation, are unavoidable, the extracted features should be invariant to these changes as far as possible.

II. For good recognition accuracy, the features should be as discriminative as possible. 
III. Since the recognition system is expected to work in the large-scale identification mode, the execution of feature extraction and matching should be as fast as possible, and the feature size should be as small as possible.

B. Given low-resolution palmprint images, how should we construct the feature representation for template protection system with the following requirements?

I. Since we adopt the Helper Data Scheme (HDS) for template protection, the feature representation should be a binary string of fixed-length.

II. For a given palmprint subject, the extracted binary strings from multiple samples generate bit errors due to the unavoidable intra-class variations. In order to maximize the allowed size of secret key for higher security, the extracted bits should be as reliable as possible, $i$. e. the probability of bit errors should be as low as possible.

III. For good verification accuracy, the binary feature representation should be as discriminative as possible.

IV. Given the HDS system, the template similarity measurement is implemented in the Hash domain. Thus, the alignment operation for matching between templates is limited.

\subsection{Overview of the thesis}

This thesis is based on published papers. The main chapters are Chapters 2-7. Each of them consists of one or more papers in their original published format. Only trivial corrections have been applied for better linguistic expression, which do not influence the contents of the paper.

\subsubsection{Main contributions}

In accordance to the research objectives introduced in Section 1.3.3, the contributions of this thesis consist of two parts: Part A. Real-valued invariant feature extraction; Part B. Binary reliable feature extraction. Table 1.2 lists the main contributions of the thesis in association with the contribution part and thesis chapters. In Figure 1.6, the block diagram of the contributions is 
Table 1.2: Main contributions of this thesis.

\begin{tabular}{|c|c|c|}
\hline Objectives & Thesis & Main Contributions \\
\hline \multirow{4}{*}{$\begin{array}{l}\text { Part } \mathbf{A} . \\
\text { Real-valued } \\
\text { invariant feature } \\
\text { extraction }\end{array}$} & Chapter 2 & $\begin{array}{l}\text { Region Covariance Matrices (RCM) as repre- } \\
\text { sentation based on Log-scaled Gabor magni- } \\
\text { tude and Gabor phase responses }\end{array}$ \\
\hline & Chapter 3 & $\begin{array}{l}\text { Mean and standard deviation as representa- } \\
\text { tion based on Log-scaled Gabor magnitude re- } \\
\text { sponses }\end{array}$ \\
\hline & Chapter 4 & $\begin{array}{l}\text { Local pattern histogram statistical represen- } \\
\text { tation based on the complex directional filter } \\
\text { bank (CDFB) transform and local binary pat- } \\
\text { tern (LBP) operation. }\end{array}$ \\
\hline & Chapter 5 & $\begin{array}{l}\text { Fourier spectral representation based on Ga- } \\
\text { bor phase coding }\end{array}$ \\
\hline \multirow[t]{2}{*}{$\begin{array}{l}\text { Part B. Binary } \\
\text { reliable feature } \\
\text { extraction }\end{array}$} & Chapter 6 & $\begin{array}{l}\text { Binary representation by one-bit quantization } \\
\text { on the features from Log-scaled Gabor magni- } \\
\text { tude and Gabor phase responses }\end{array}$ \\
\hline & Chapter 7 & $\begin{array}{l}\text { Binary representation by multi-bit equal- } \\
\text { probability-interval quantization on the fea- } \\
\text { tures from Log-scaled Gabor magnitude re- } \\
\text { sponses }\end{array}$ \\
\hline
\end{tabular}

presented in the context of a system diagram. Based on the system operating process, Part A involves three steps: i. Multi-scale and multi-orientational transform; ii. Region-based statistical feature extraction or pixel-based transform feature extraction; iii. Feature reduction. Part B consists of two procedures: i. Real-valued feature extraction; ii. Quantization and bit selection.

\subsubsection{Chapters overview}

The thesis is organized as follows:

In Chapter 2, the basic idea of the region covariance matrix (RCM) as feature descriptor is introduced. Assuming that the covariance of a distribution is able to discriminate it from other distributions, a region can be represented by a covariance matrix. It is an open issue how to create a suitable feature mapping for each pixel so that the generated RCM in a region can be discriminative and robust to illumination variations and image translation and rotation. In this chapter, we will introduce a new method to create the feature mapping based on the Gabor magnitude and phase responses. The palmprint image needs to be partitioned into several regions. For each region, one RCM can 


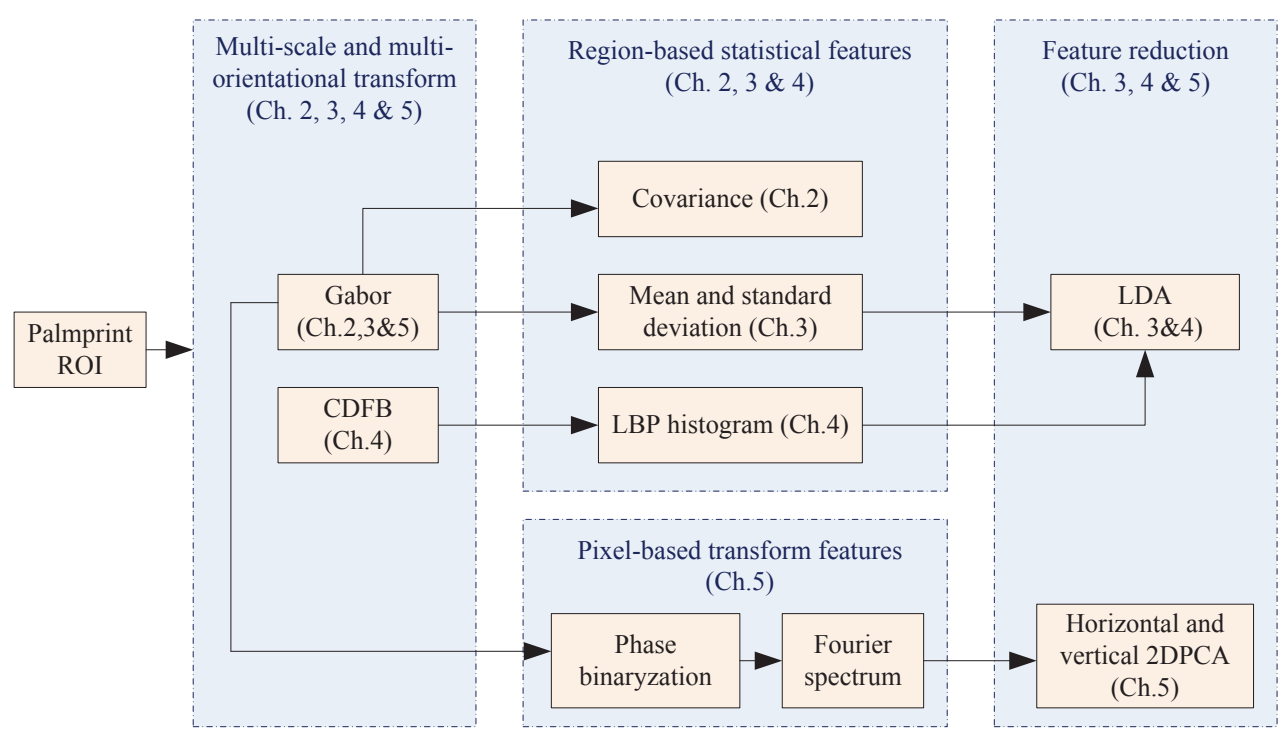

(a) Part A: Real-valued invariant feature extraction for the unprotected palmprint recognition system.

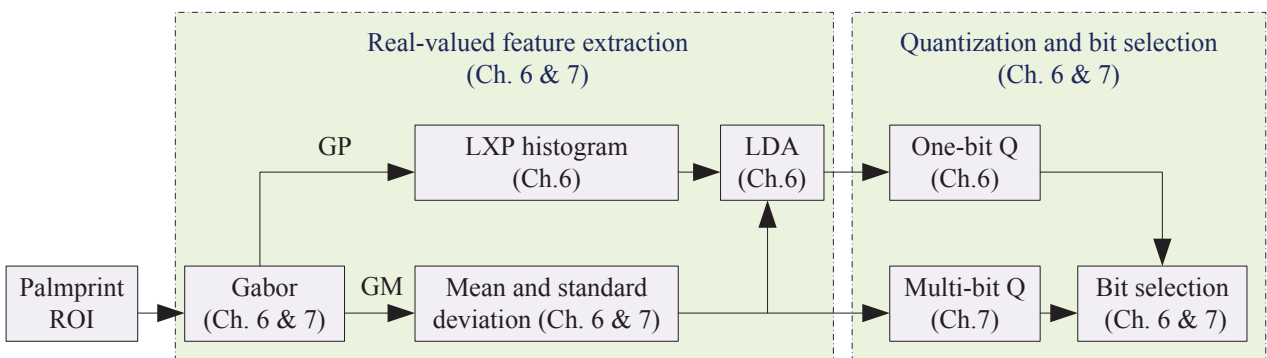

(b) Part B: Binary reliable feature extraction for the palmprint template protection system.

Fig. 1.6: Block diagram of our designed system for the research objectives Parts A and $\mathrm{B}$ respectively, focusing on the main contributions of this thesis. (ROI - region of interest; LBP - local binary pattern; LDA - linear discriminant analysis; 2DPCA - twodimensional principle component analysis; LXP - local xor pattern; Q - quantization) 
be obtained based on the proposed feature mapping function for each pixel. Finally, the obtained several RCMs are combined as the proposed palmprint representation. Especially, the Gabor magnitude is log-scaled for more powerful discriminative ability.

In Chapter 3, we further investigate the region-based statistical features for palmprint representation based on the Gabor filtering. In our experiments it is found that the log-scaled Gabor magnitude response in each filtered subband approximates a Gaussian distribution. Assuming that the mean and standard deviation of a Gaussian distribution is able to discriminate it from other Gaussian distributions, we propose to extract the mean and standard deviation values to create the feature vector. It is expected to be robust against image translation and rotation. Based on the generated feature vector, the linear discriminant analysis (LDA) is carried out to enhance the recognition performance.

In Chapter 4, the directional filter bank (CDFB) is explored to transform the palmprint image. Then the local binary pattern (LBP) is operated on the transformed coefficients. The generated LBP histograms are the region-based features, which leads to the proposed palmprint representation after being processed by the LDA. The comparisons between the CDFB and the other multi-scale and multi-orientational transforms are given in terms of recognition rate, storage requirement and computational complexity, including Gabor filter bank, dual-tree complex wavelet transform (DTCWT), discrete Contourlet transform and Nonsubsampled Contourlet transform (NSCT). In addition, the comparisons between the proposed method and the currently reported several local Gabor binary pattern methods are given.

In Chapter 5, the coding-based methods are studied, which are reported for the palmprint verification system with high discriminative ability and robustness to illumination variations. The drawback of these methods is that the generated code features are sensitive to image translation and rotation. Inspired by their merits and drawbacks, we propose a new representation method based on the classical Palm Code method, which encodes the Gabor phase into bits pixel by pixel. The pixel-based phase code features are sensitive to image translation. Therefore, we transform the phase code into its Fourier domain, since a shift in the time domain causes no change in its Fourier magnitude spectrum. Furthermore, the horizontal and vertical two-dimensional principle component analysis $\left((2 \mathrm{D})^{2} \mathrm{PCA}\right)$ is implemented, which can not only reduce the feature dimension but also lead to translation invariance.

In Chapter 6, we present a binary presentation method for palmprint tem- 

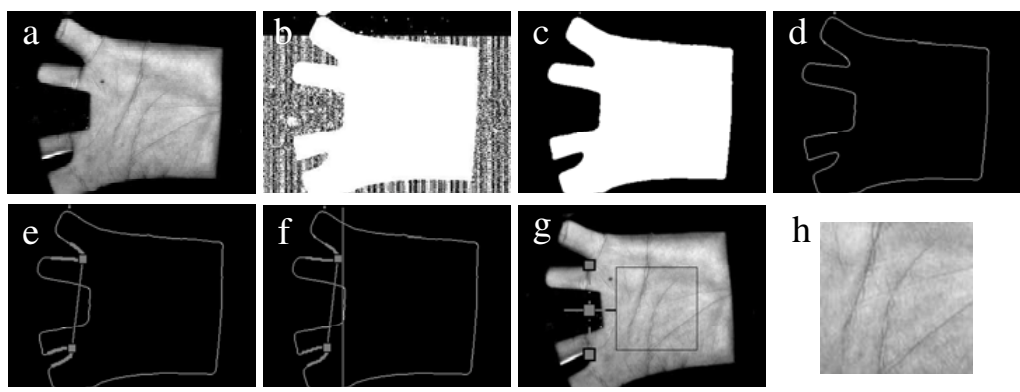

Fig. 1.7: A typical palmprint image in HongKong Polytechnic University (PolyU) Palmprint Database, and the corresponding ROI extraction processing. (a) original image, (b) Gaussian lowpass filtering, (c) binarization and median filtering, (d) boundary tracking by Sobel operating, (e) reference points locating, (f) rotation angle computing, (g) image rotating, and (h) extracted ROI.

plate protection system. The verification error rate (FRR/FAR) and the bit error rate (BER) of genuine templates are mainly considered as the performance indicator. The proposed method explores the region-based features extracted from the Gabor magnitude (GM) and Gabor phase (GP) responses respectively. In order to generate the bits of identically and independently distribution, the linear discriminant analysis (LDA) is adopted, which contributes to the good verification accuracy as well. The generated real-valued features are quantized into bits by a one-bit quantizer. For achieving a binary string of lower BER, bits are selected based on the absolute difference between the real value and the quantization threshold.

In Chapter 7, a binary representation is proposed for palmprint template protection system. Assuming that the Helper Data Scheme (HDS) with a BCH error correcting code is implemented, this chapter targets a binary representation which can achieve a low FRR/FAR and allows a long secret key to be combined. To reduce the likelihood of a key being obtained by an attacker's guesses, we expect that the allowed key length can be larger than 70 . The allowed maximum key length not only depends on the BER performance of genuine binary strings, but also on the code length and the error-correcting ability of the used error correcting code (ECC). The real-valued features for quantization is the region-based GM statistical features. A multi-bit equalprobability-interval quantization method is adopted. The reliable bits are selected based on a detection rate optimized bit allocation (DROBA) principle. 


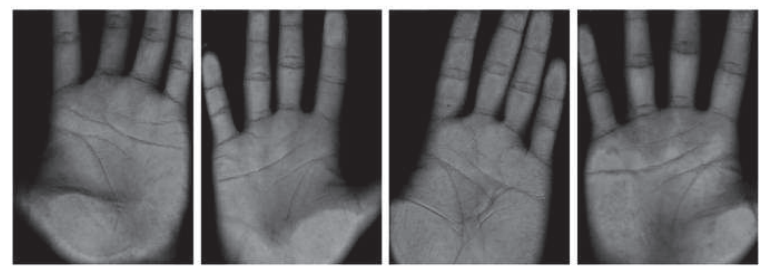

Fig. 1.8: Some original images in Beijing Jiaotong University Palmprint Database.

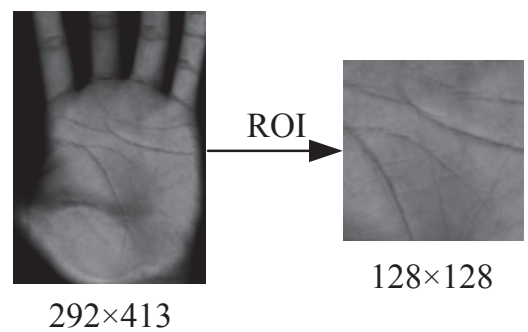

Fig. 1.9: An original palmprint image in Beijing Jiaotong University Palmprint Database and its corresponding ROI.

\subsubsection{Biometric data sets}

In this thesis, two palmprint databases are involved for performance evaluation: (1) HongKong Polytechnic University (PolyU) Palmprint Database and (2) Beijing Jiaotong University Palmprint Database (BJTU_PalmprintDB (V1.0)). However, the HongKong PolyU Palmprint Database is used in most of cases, since it is considered as the de facto standard for evaluating the palmprint recognition technologies.

HongKong Polytechnic University (PolyU) Palmprint Database: It contains 7752 grayscale images in BMP format. They are from 386 palms (including left and right palms) captured by a CCD-based device. The image resolution is 75 dpi. The image collection is done in two sessions, which differ in illumination conditions. One palm provides around ten samples respectively in each session. The average interval between the first and second session is two months. An original image size of $384 \times 284$ pixels is shown in Figure 1.7(a). For the ROI extraction, we implement a sequence of image processing procedures as Figure 1.7(b)-(g) illustrate. Figure 1.7(h) shows the extracted ROI size of $128 \times 128$.

Beijing Jiaotong University Palmprint Database (BJTU_PalmprintDB (V1.0)): it contains 3460 grayscale images in BMP format corresponding to 346 palms. 
They are captured by a Fujitsu fi-60F high speed digital scanner. The original image is size of $292 \times 413$. The resolution is $72 \mathrm{dpi}$. The images are from 173 volunteers from the students and staff in Beijing Jiaotong University. For each subject, ten samples are collected from the left and right palms respectively. Several image samples, size of $292 \times 413$, are shown in Figure 1.8. The extracted ROI is size of $128 \times 128$, as it shows in Figure 1.9. Its preprocessing method is proposed in [15]. 
Chapter 2

\section{Region covariance matrices}

\subsection{Chapter introduction}

PURPOSE. This chapter introduces a Region Covariance Matrix (RCM) representation for palmprint recognition which is real-valued. The investigated multi-scale and multi-orientational transform is a Gabor filter bank. The proposed representation is based on the regional statistical features. Assuming that the covariance of a distribution is able to discriminate it from other distributions, a region can be represented by a covariance matrix. Since the mean of region is reduced during the covariance calculation, the RCM leads to illumination invariance to some extent. Further, RCM is a symmetric matrix. Its entries have no information regarding the pixels' order and number of the considered region, so that RCM representation is expected to be scale and rotation invariance, unless the features for each pixel include some orientation information. Therefore, we resort to a group of RCMs for palmprint representation. The open issue is how to create a suitable feature mapping for each pixel so as to generate a RCM of high discriminative ability. This chapter presents our proposed novel approach.

CONTENTS. Firstly, a brief review of RCM as descriptors is given in Section 2.2.3. Some feature mapping functions are introduced which have been reported to achieve great success in object tracking and texture classification. 


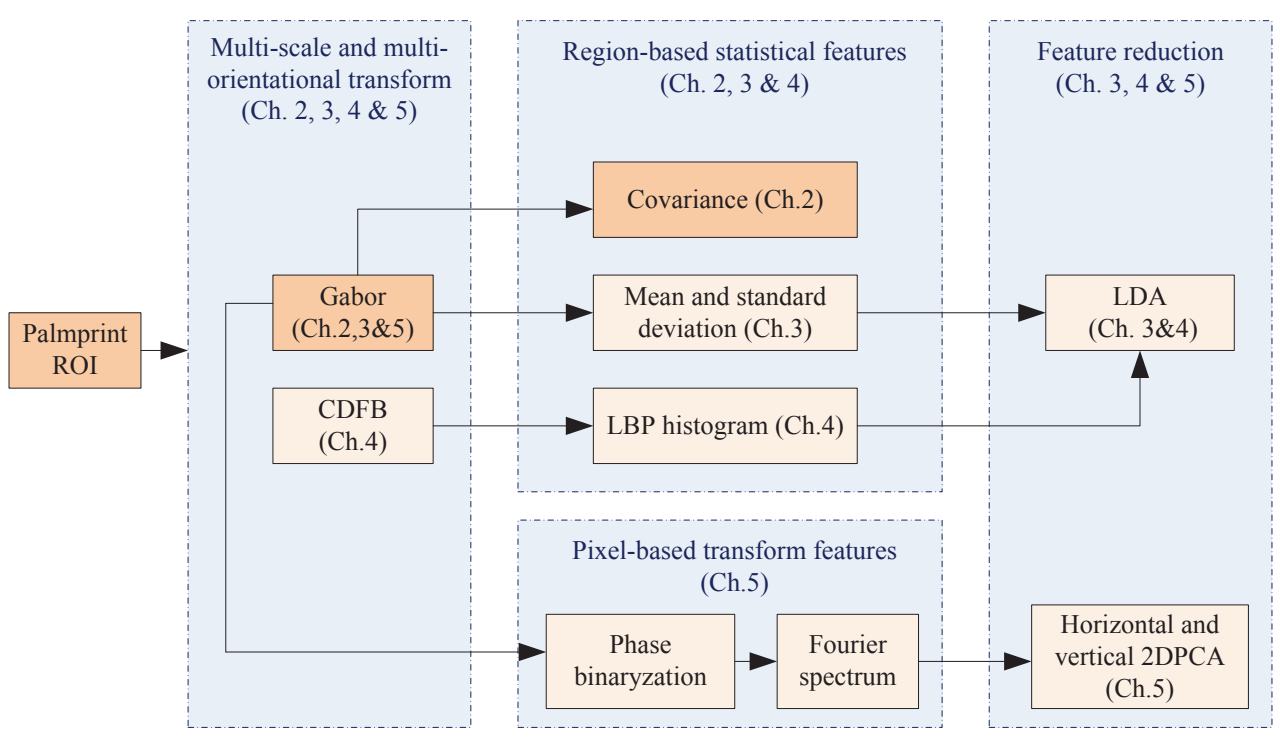

Fig. 2.1: Block diagram of our designed system, highlighting the context of Chapter 2 and its referred blocks.

Then, in Section 2.2.4 our proposed feature mapping method for RCM representation is presented, which is based on the multi-scale and multi-orientation Gabor filtered coefficients. The Gabor magnitude and phase responses are both exploited. Especially the used Gabor magnitude is in log-scale so that the log-scaled Gabor magnitude distribution is close to Gaussian. Accordingly, the resulted RCM representation from our proposed feature mapping method turns out to be more discriminative. Thirdly, we evaluate our algorithm on the HongKong PolyU Palmprint Database. In the context of system diagram, the content of this chapter and its referred blocks are highlighted in Figure 2.1.

PUBLICATION(s). The content of Section 2.2 of this chapter has been published in [16]. 


\subsection{Region covariance matrices as representation}

\subsubsection{Abstract}

Region covariance matrices (RCMs) as feature descriptors have been developed due to the advantages of low dimensionality, being scale and illumination independent. How to define a feature mapping vector for the RCMs construction of strong discriminative ability is still an open issue. In this paper, there is a focus on finding a more efficient feature mapping vector for RCMs as palmprint descriptors based on Gabor magnitude and phase (GMP) information. Specially, Gabor magnitude (GM) features of each palmprint image approximate a lognormal distribution. For palmprint recognition, the logarithmic transformation of GM proves to be important for the discriminative ability of corresponding RCMs. All experiments are performed on the public Hong Kong Polytechnic University (PolyU) Palmprint Database of 7752 images. The results demonstrate the efficiency of our proposed method, and also show that adding pixel locations and intensity components to the feature mapping vector has a negative effect on palmprint recognition performance for our proposed Log_GMP based RCM method.

\subsubsection{Introduction}

Nowadays, with the rapid development of internet and information technologies, our life is becoming more efficient and easier. However, these new technologies also pose a challenge to keep personal information. There is an urgent need to thoroughly revamp the system of personal identification and verification as used by our government agencies and commercial organizations [17]. As an efficient and safe solution to such schemes, biometrics technology has recently been receiving wide attention from researchers. It concerns with identifying people by their physiological characteristics such as face, fingerprint, iris and hand geometry or some behavioral specialities like voice, stepping pattern, signature and gesture [18-27]. To make a system more secure, researchers often combine different biometric methods together, e. $g$. face and voice, face and fingerprint, or face and hand geometry [28-30]. However, multiple biometrics usually have to use multiple sensors, which limits its range of application. Comparatively speaking, one of the new technologies is the palmprint based recognition, since its distinguished characteristics including stable structures, 
low-cost and low-intrusiveness [31]. Various features, including principal lines, wrinkles, ridges, minutiae points, singular points and texture, can be extracted at different image resolutions [8]. Early studies focus on palmprint structure features for off-line palmprint images of high resolution (up to $500 \mathrm{dpi}$ ) $[32,33]$. Nevertheless, for civil and commercial applications, the low-resolution palmprint images (less than $100 \mathrm{dpi}$ ) captured by CCD-based device or digital scanner are more suitable than high-resolution images because of their smaller file sizes, which results in shorter computation times during preprocessing and feature extraction [8]. Extracting structural features from the low-resolution palmprint images becomes much more difficult, and the mere principal lines do not contribute adequately to high accuracy [12]. Until now, it is still a key issue for palmprint recognition to extract the representation effectively from low resolution image.

Due to the simplicity and efficiency for the feature extraction and representation, subspace transform based methods, such as Eigenpalms [34] and Fisherpalms [35], have been studied extensively. However, these methods suffer from image variations in illumination, rotation and translation. The research on coding based methods, which encodes the response of a bank of filters, is another active area. Zhang and Kong et al. [8] proposed PalmCode, which encodes the phase of the filter responses as bitwise features. Subsequently, Kong et al. [36] used fusion rule at feature layer to further improve PalmCode. And this approach was named as FusionCode. Besides the phase information, the palmline orientation information is also popularly coded for feature representation due to its advantages of stability, robustness to illumination variation and fast implementation. Such schemes include competitive code (CompCode) [37], palmprint orientation code (POC) [38], robust line orientation code (RLOC) [39] and so on. These algorithms share a common strategy: several filters or masks with different orientation are convolved with the image, and then the "dominant" orientation is determined with certain competitive rule. For instance, CompCode and its recent improved editions (ImprovedCompCode [40] and BOCV [41]) apply a bank of Gabor filters, while POC uses self designed masks and RLOC does the modified finite Radon transform. In addition, Wu et al. [42] proposed a DoG code method which first convolves the image using 2D Gaussian filter and then encodes the zero-crossing information of horizontal and vertical gradient values, respectively. However, these code representation based methods require the alignment of the corresponding pairs of pixels ideally for palmprint matching. Though they can solve this problem by vertically and horizontally translating feature-planes to construct an enlarged training set, it is highly time-consuming in this way to determine the identity of one test palmprint, especially in a large registered database. 
In order to be robust to image variations, palmprint texture energy based representation approaches have been introduced and deemed to be the promising methods. Li et al. [43] used four masks to highlight the distribution of line segments in horizontal, vertical and two diagonal lines, and then computed the global and local energies to represent a palmprint image. Wu et al. [44] introduced wavelet energy feature (WEF), which is robust to some extent in rotation and translation of the images. Recently, the Gabor filters, whose kernels are similar to the response of the two-dimensional receptive field profiles of the mammalian simple cortical cell, exhibiting the desirable characteristics of spatial locality, spatial frequency and orientation selectivity $[45,46]$, so often act as a powerful tool to extract the main features from palmprint images. Laadjel et al. [47] convolved the palmprint image with 32 Gabor filters of different scales and orientations, resulting in an overwhelming highdimensional feature space. In order to reduce the dimension of the feature vector, a popular strategy is to down-sample the filtered images with a factor. In Ref. [47], the down-sampled Gabor features were concatenated to form an augmented feature vector, which was then projected into a low-dimensional linear subspace by Linear Discriminant Analysis (LDA). Similar work can be found in Ref. [48], which conducted two-step 2DPCA to reduce the dimension of Gabor feature space. The disadvantage of down-sampling is that a great number of discriminative Gabor features are discarded. For overcoming the global disturbance occurred on palmprint images, Pan et al. [49] divided the Gabor filtered image into two-layered partitions and then calculated the difference of standard deviation between each lower-layer sub-block and its resided upper-layer block (called local relative variance). Additionally, Chu et al. [50] employed the Adaboost algorithm to select the most discriminative Gabor features. However, it is very time-consuming to select the most useful ones from so many Gabor features.

Recently, region covariance matrices (RCM), serving as a feature descriptor framework, has been developed and employed in object detection and tracking with promising results [51,52]. The RCM in Refs. [51] and [52] is a covariance of simple features including coordinate, color, the first-order gradient, and the second-order gradient. However, Pang et al. [53] found that the discriminative ability of origin RCMs was inadequate to effectively recognize human faces. To address this issue, Pang et al. [53] proposed to construct RCMs of pixel locations, intensity component and Gabor magnitude (GM) features, which is denoted as Gabor-based RCM (GRCM) algorithm, achieving desirable face recognition performance. Subsequently, Lu et al. [54] introduced an enhanced GRCM feature extraction method (EGRCM) by constructing two group of RCMs utilizing both Gabor magnitude (GM) and Gabor phase 
(GP) information respectively and conducting the classification with a sum rule. However, how to develop more effective feature mapping functions to construct the RCMs for recognizing palmprint is still an open issue.

In this paper we will conduct more experiments to find a more effective feature mapping vector for palmprint recognition. The rest of the paper is organized as follows: Sec. 2.2.3 describes the recently related work on the RCM as feature descriptors. Our proposed approach to construct RCMs for recognizing palmprint is given in Sec. 2.2.4. Sec. 2.2.5 will show the experimental results on the PolyU Palmprint Database of 7752 images. The paper is concluded with some closing remarks in Sec. 2.2.6.

\subsubsection{Related work}

The original RCM proposed by Tuzel et al. [51] is a matrix of covariance of several image statistics computed inside a region of an image. The RCM matrix is considered as a feature descriptor of the region. Classification is conducted based on these RCMs.

Let $I$ be an intensity image of size $W \times H$. Define a function $\phi(I, x, y)$ that extracts $d$ dimensional feature vector $z_{i}$ from a pixel at $(x, y)$ of $I, i$. e. ,

$$
\phi(I, x, y)=z_{i} \in \Re^{d} .
$$

where $i=y \times W+x$ is the index of $(x, y)$. The function $\phi$ can be any mapping such as intensity, color, gradients, filter response, etc. . Let $F$ be the $W \times H \times d$ dimensional feature image extracted from $I$. Considering a region $R \subset F$, the number of pixels in the region $R$ is $n$. Then the region $R$ can be represented by the $d \times d$ covariance matrix of the feature points $\left\{z_{i}\right\}_{i=1, \ldots, n}$ inside the region

$$
C_{R}=\frac{1}{i-1} \sum_{i=1}^{n}\left(z_{i}-u_{R}\right)\left(z_{i}-u_{R}\right)^{T} .
$$

where $u_{R}$ is the mean of the points $\left\{z_{i}\right\}_{i=1, \ldots, n}$ in region $R$.

A region can be represented by a covariance matrix $C_{R}$ which is based on the assumption that the covariance of a distribution is enough to discriminate it from other distributions. For the extracted feature image $F$, each pixel has $d$ 
features. The diagonal entries of $C_{R}$, which is a symmetric matrix, represent the variance of each feature, and the non-diagonal entries represent the correlations between any two features. Since $C_{R}$ does not have any information regarding the order and the number of pixels in the considered region $R$, it implies a certain scale and rotation invariance over the regions in different images, unless the feature represents orientation information of the points. Further, the mean $u_{R}$ of the points in region is reduced during the covariance computation as shown in Eq. (2.2), which leads to illumination invariance to some extent.

The previous work has proved that suitable feature mapping function $\phi(I, x, y)$ is crucial to RCM. In Ref. [51], for gray level images, $\phi(I, x, y)$ is defined as:

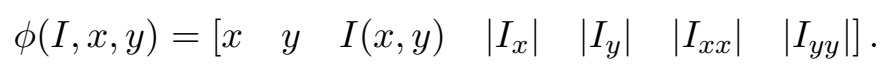

where $x$ and $y$ denote pixel locations. $I(x, y)$ is the intensity component with respect to $x$ and $y . I_{x}$ and $I_{x x}$ are the first and second order derivatives. $|\cdot|$ denotes the absolute operator. This feature mapping function has achieved great success in object tracking and texture classification $[51,52]$. However, the recognition rates of this RCM-based algorithm are very low when being applied to face recognition [53]. In Ref. [53], Gabor magnitude (GM) features are used for the construction of RCMs, achieving better recognition performance. When Gabor functions with four orientations and five scales are chosen, the dimension of each Gabor feature is $4 \times 5=20$. Then the mapping of GM based RCM is defined as:

$$
\phi_{G M}=\left[\begin{array}{llll}
x & y & I(x, y) & m_{0,0}(x, y) \ldots m_{3,4}(x, y)
\end{array}\right]
$$

More recently, Lu et al. [54] enhanced GM-based RCM algorithm for palmprint recognition by introducing the mapping of Gabor phase (GP) based RCM, which is defined as:

$$
\phi_{G P}=\left[\begin{array}{llll}
x & y & I(x, y) & p_{0,0}(x, y) \ldots p_{3,4}(x, y)
\end{array}\right]
$$

In Ref. [54], the GM-RCMs and GP-RCMs are constructed respectively. Finally, classification is conducted with the sum rule based on the GM-RCMs 
and GP-RCMs.

Inspired by the advantages including illumination and scale invariance of using covariance matrices as region descriptor, we focus on finding a more effective feature mapping function $\phi(I, x, y)$ for palmprint recognition task based on the RCMs framework.

\subsubsection{Log_GMP based RCMs as palmprint descriptors}

The Gabor wavelet representation captures salient visual properties including spatial localization, orientation selectivity, and spatial frequency characteristic [46]. In addition to accurate time-frequency location, they also provide robustness against varying brightness and contrast of images. A circular 2D Gabor function has the following general form [12]:

$$
\begin{aligned}
G(x, y, \theta, u, \sigma)= & \frac{1}{2 \pi \sigma^{2}} \exp \left\{\frac{-\left(x^{2}+y^{2}\right)}{2 \sigma^{2}}\right\} \\
& \times \exp \{2 \pi i(u x \cos \theta+u y \sin \theta)\} .
\end{aligned}
$$

where $i=\sqrt{-1}, u$ is the frequency of the sinusoidal wave, $\theta$ controls the orientation of the function, and $\sigma$ is the standard deviation of the Gaussian envelope. In practice, a Gabor function $G(x, y, \theta, u, \sigma)$ with a special set of parameters $(\theta, u, \sigma)$, is transformed into a discrete Gabor filter. In order to provide more robustness to brightness, the Gabor filter is turned to zero DC (direct current) with the application of the following formula [12]:

$$
\begin{aligned}
\mathbf{G}(x, y, \theta, u, \sigma)= & G(x, y, \theta, u, \sigma) \\
& -\frac{\sum_{i=-n}^{n} \sum_{j=-n}^{n} G(i, j, \theta, u, \sigma)}{(2 n+1)^{2}} .
\end{aligned}
$$

where $(2 n+1)^{2}$ is the size of the filter. Modified from the experimental results of Kong et al. [12], we design a Gabor function of five scales and four 
orientations for more robust features, whose parameters are set as follows:

$$
\begin{aligned}
& u_{v}=0.2592 / \sqrt{2^{v}}, v=0,1, \ldots, 4 \\
& \theta_{k}=\pi k / 4, k=0, \ldots, 3
\end{aligned}
$$

For a given image $I(x, y)$, the convolution of $I(x, y)$ and a Gabor filter is defined as follows:

$$
O_{v, k}(x, y)=I(x, y) * \mathbf{G}(x, y, \theta, u, \sigma) .
$$

The Gabor magnitude (GM) part is defined as:

$$
m_{v, k}(x, y)=\sqrt{\left(\operatorname{Re}\left(O_{v, k}(x, y)\right)\right)^{2}+\left(\operatorname{Im}\left(O_{v, k}(x, y)\right)\right)^{2}}
$$

The Gabor phase (GP) is defined as:

$$
p_{v, k}(x, y)=\arctan \frac{\operatorname{Im}\left(O_{v, k}(x, y)\right.}{\operatorname{Re}\left(O_{v, k}(x, y)\right.} .
$$

where $\operatorname{Re}\left(O_{v, k}(x, y)\right.$ and $\operatorname{Im}\left(O_{v, k}(x, y)\right.$ denote the real and imaginary parts of $O_{v, k}(x, y)$.

Next, we consider computing the covariance matrices as the feature descriptors using the Gabor features. As described in Sec. 2.2.3, the covariance matrices $C_{R}$ is a symmetric matrix, and the diagonal entries of $C_{R}$ represent the variance of each feature while the non-diagonal ones represent the correlations between any two features in the given region $R$. Since the variance information of each feature is covered in the feature descriptor of RCMs, it is indispensable to observe the distribution of each feature. If the feature is far from Gaussian, the representation using the variance information will lead to information loss to some extent.

Figure 2.2 shows the Gabor wavelet representation (GM and GP) of a sample image at five scales and four orientations with the following parameters: $\sigma=5.6176, u_{v}=0.2592 / \sqrt{2^{v}}, v=0,1, \ldots, 4, \theta_{k}=\pi k / 4, k=0, \ldots, 3$. Now, 


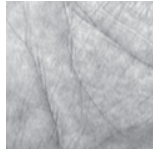

(a) Original image.
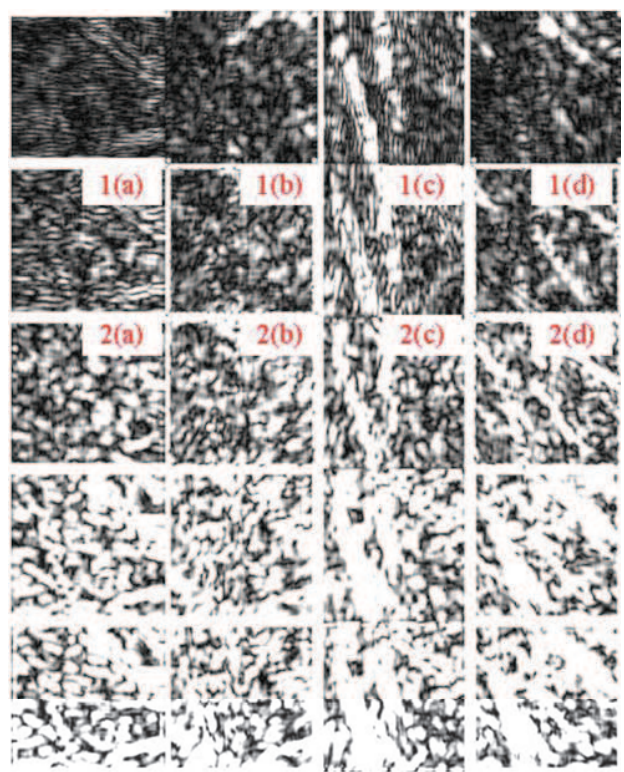

(b) The Gabor magnitude (GM) part of the representation.

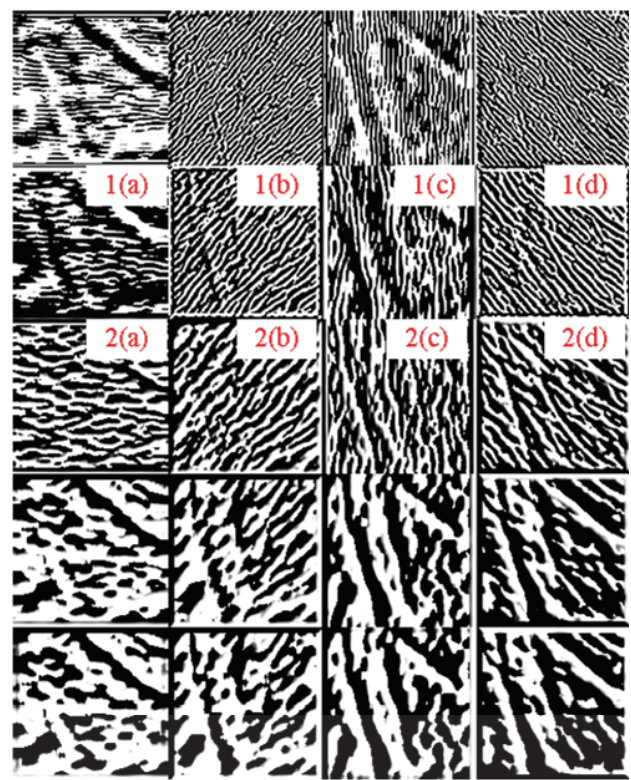

(c) The Gabor phase (GP) part of the representation.

Fig. 2.2: The Gabor representation of a sample image at five scales and four orientations with the following parameters: $\sigma=5.6176, u_{v}=0.2592 / \sqrt{2^{v}}, v=0,1, \ldots, 4$, $\theta_{k}=\pi k / 4, k=0, \ldots, 3$. 

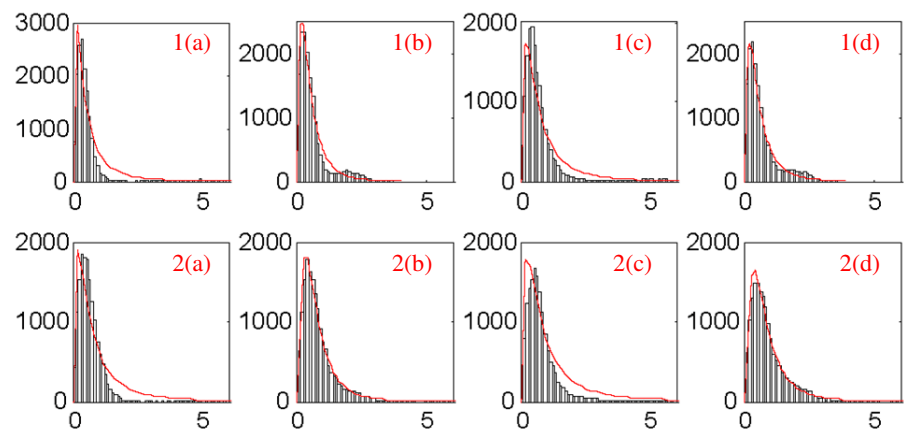

Fig. 2.3: The histograms of eight GM Gabor responses labeled in Fig. 2.2(b), together with the fitted lognormal density using the Maximum Likelihood Estimate (MLE) algorithm.
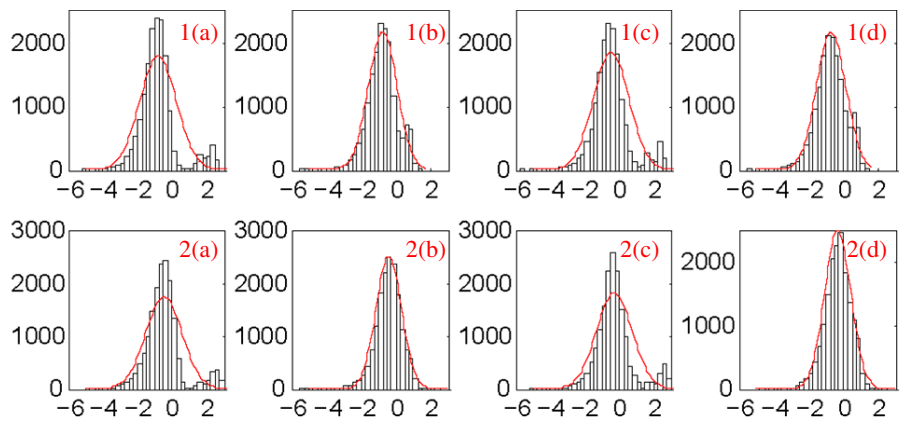

Fig. 2.4: The histograms of eight LogGM responses corresponding to Fig. 2.3, together with the fitted normal density using the Maximum Likelihood Estimation (MLE) algorithm.
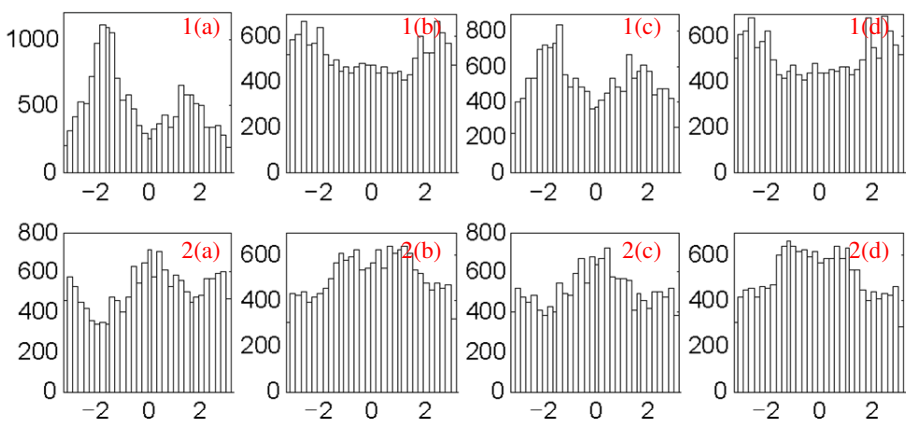

Fig. 2.5: The histograms of eight GP Gabor responses labeled in Fig. 2.2(c). 
we empirically assess the characteristics of GM and GP distributions respectively. The GM histograms of eight Gabor responses labeled by 1(a)-1(d), and 2(a)-2(d) in Fig. 2.2 are shown in Fig. 2.3, together with the fitted lognormal distribution using the Maximum Likelihood Estimation (MLE) algorithm. We can see that the lognormal distribution fits the GM response well by and large. By the logarithmic transformation of GM, we can achieve LogGM, which can be formulated as $\log G M_{v, k}=\log \left(m_{v, k}(x, y)\right)$. The eight LogGM histograms corresponding to GM responses in Fig. 2.3 are shown in Fig. 2.4, together with the fitted Gaussian distributions respectively. From Figs. 2.3 and 2.4, we can conclude that palmprint GM features approximate a lognormal distribution, i. e., LogGM is a Gaussian approximation distribution. Further, the corresponding eight GP histograms are also investigated, shown in Fig. 2.5, from which we can conclude that GP distribution is far from Gaussian. However, that is not to say GP information is insignificant. In fact, it has been proved useful in Ref. [54] to construct a covariance matrix by GP information (as shown in Eq. (2.5)), which utilizes the variance of each GP mapping and the correlations between any two GP mappings. Nevertheless, the correlation of GM and GP is ignored.

Based on the both consideration of feature's distribution and the correlation of GM and GP, we define a new feature mapping function $\phi_{L_{o g} G M P}$ formulated as follow:

$$
\phi_{\log _{-} G M P}=\left[\log \left(m_{v, k}(x, y)\right) \quad p_{v, k}(x, y)\right] .
$$

where $v=0,1, \ldots, 4, k=0, \ldots, 3$, and the vector length $d$ of each feature point is 40. Substitute Eq. (2.12) into Eq. (2.2), we get a new Gabor-based RCM in region $R$, which can be denoted by $C_{L_{\text {Log_GMP }}}$ for notational simplicity. Obviously, the diagonal entries of $C_{L_{\text {og }} G M P}$ includes the variances of $\log \left(m_{v, k}(x, y)\right)(\log G M)$ and $p_{v, k}(x, y)(\mathrm{GP})$ respectively. The non-diagonal entries consist of the correlations between any two LogGM features, the correlations between any two GP features, and also the ones between any LogGM and GP features. The construction process is very different with the one proposed in Ref. [54], which forms $\phi_{G M}$ and $\phi_{G P}$ respectively, i. e. one region will be presented by two covariance matrices. Here, the size of $C_{L_{o g} G M P}$ is $d \times d=40 \times 40$. Due to the symmetry of $C_{L_{\text {og_GMP }} \text {, there are only }}$ $\left(d^{2}+d\right) / 2=\left(40^{2}+40\right) / 2=820$ different values. Note that Log_GMP RCM is independent of image size, scale and illumination because the feature rep-

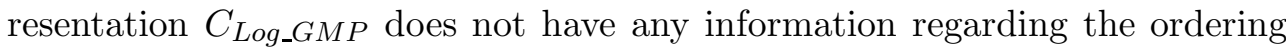
and the number of points, and the average of features is reduced during the covariance computation. In addition, noise that corrupts images is largely filtered out. 

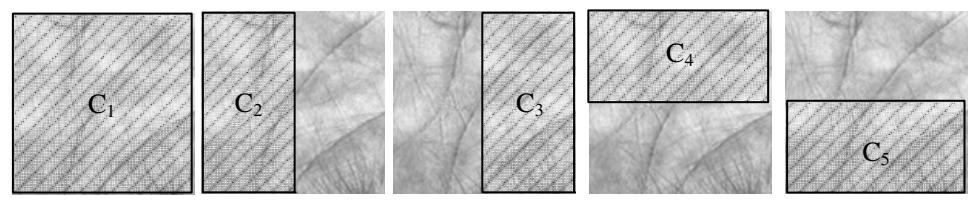

Fig. 2.6: Five regions of palmprint image, from which five corresponding RCMs are constructed.

As in Ref. [54], a palmprint is represented with five Log_GMP RCMs $\left(C_{1}, C_{2}, C_{3}, C_{4}, C_{5}\right)$ extracted from five different regions as shown in Fig. 2.6, where $C_{1}$ is global representation defined over the whole palmprint image, and the last are part-based. By combining global and part-based representation, we can increase robustness towards large image variations.

The nearest neighbor algorithm is applied here for palmprint classification. The distance measurement proposed in Ref. [51] is used for the dissimilarity measurement of two covariance matrices, which is formulated as follows:

Suppose $C_{i}^{g}$ and $C_{i}^{p}(i=1,2,3,4,5)$ are the Log_GMP RCMs from gallery and probe sets respectively, the dissimilarity distance between a gallery image $G$ and a probe one $P$ can be calculated as

$$
\rho(G, P)=\sum_{i=1}^{5} \rho\left(C_{i}^{g}, C_{i}^{p}\right) .
$$

where the distance between RCM $C_{i}^{g}$ and $C_{i}^{p}$ is computed by the eigenvaluebased distance [51]

$$
\rho\left(C_{i}^{g}, C_{i}^{p}\right)=\sqrt{\sum_{i=1}^{n} \ln ^{2} \lambda_{i}} .
$$

and $\lambda_{i}, i=\{1, \ldots, n\}$ are the generalized eigenvalues of $C_{i}^{g}$ and $C_{i}^{p}$ calculated as

$$
\lambda_{i} C_{i}^{g} u_{i}=C_{i}^{p} u_{i}, i=\{1, \ldots, n\} .
$$

A nearest neighbor classifier is used with the distance in Eq. (2.13). Our proposed method can be denoted as Log_GMP based RCMs method. 


\subsubsection{Experimental results}

The proposed method in this paper was tested on the Hong Kong Polytechnic University (PolyU) Palmprint Database. This database contains 7752 grayscale images in BMP image format, corresponding to 386 different palms. The palmprint images were collected in two occasions. Each time, the subjects were asked to provide around ten images from the left palm and ten images from the right palm. The average interval between the first and the second collection was two months. The resolution of all the original palmprint images is $384 \times 284$ pixels at $75 \mathrm{dpi}$. In all our experiments, by performing the similar preprocessing approach described in Ref. [8], palmprints were orientated and the ROI, whose size is $128 \times 128$, was cropped.

Two group of experiments are performed. First, we focus on constructing a more effective feature mapping function based on the RCM framework for palmprint representation. Second, the recognition performance comparison of our proposed approach with several current state-of-the-art palmprint recognition methods is given in Sec. 2.2.5.2. All the experiments of our work are executed on a personal computer with an Intel Pentium(R) Dual-Core Processor(E5200@2.50GHz) and 2GB RAM configured with Microsoft Windows $\mathrm{XP}$ and Matlab 7.5. In all the experiments, we randomly select three images of each class to construct the enrollment set and the last for test, $i$. e. the enrollment set and the test set contain 1158 and 6594 images respectively. To enhance the accuracy of the assessment, ten runs of such a partition are executed. The average recognition accuracies of each evaluated method over the ten splits are tabulated.

\subsubsection{Constructing a more effective feature mapping function}

In this group of experiments, we focus on constructing a more effective feature mapping function based on the RCM framework for palmprint representation by examining the contribution of the correlation information of Log_GM and GP, and the effect of logarithmic transformation of Gabor magnitude (GM) for recognition rate.

Table 2.1 lists the recognition rates when using GM or GP information separately to construct RCMs. One can find that GM and GP information are both powerful to discriminate palmprint image, with the recognition rates $95.37 \%$ and $94.90 \%$ respectively. The logarithmic transformation of GM (Log_GM) 
Table 2.1: Recognition accuracy comparison when using GM or GP information separately to construct RCM.

\begin{tabular}{|l|l|c|}
\hline Method & $\phi(I, x, y)$ & Accuracy(\%) \\
\hline \hline GM & {$\left[m_{v, k}(x, y)\right]$} & 95.37 \\
Log_GM & {$\left[\log \left(m_{v, k}(x, y)\right)\right]$} & 98.01 \\
GP & {$\left[p_{v, k}(x, y)\right]$} & 94.90 \\
\hline
\end{tabular}

Table 2.2: Recognition accuracy comparison when considering the correlation between GM and GP to construct RCM.

\begin{tabular}{|l|l|c|}
\hline Method & $\phi(I, x, y)$ & Accuracy $(\%)$ \\
\hline \hline GM_GP & {$\left[m_{v, k}(x, y)\right]$ and $\left[p_{v, k}(x, y)\right]$} & 97.18 \\
GMP & {$\left[m_{v, k}(x, y) \quad p_{v, k}(x, y)\right]$} & 97.42 \\
Log_GM_GP & {$\left[\log \left(m_{v, k}(x, y)\right)\right]$ and $\left[p_{v, k}(x, y)\right]$} & 98.60 \\
Log_GMP & {$\left[\log \left(m_{v, k}(x, y)\right) \quad p_{v, k}(x, y)\right]$} & 99.29 \\
\hline
\end{tabular}

based RCM achieves $98.01 \%$ recognition rate, which obviously outperforms GM with higher recognition rate of $2.64 \%$. Thus, the logarithmic transformation operation of GM is very important in these RCM-based methods.

Table 2.2 shows the comparison among four feature mapping functions which exploit both GM and GP information to construct RCMs. GM_GP constructs two covariance matrices as feature descriptor while GMP forms one covariance matrix with the non-diagonal terms including the correlation information between GM and GP. By comparing the recognition rates between GM_GP and GMP, or Log_GM_GP and Log_GMP, we can conclude that it can strongly enhance the discriminative ability of the corresponding RCMs by taking the correlation information of Log_GM and GP into account.

To verify the contributions of pixel locations $(x, y)$ and intensity component $I(x, y)$, which are both used in Ref. [53] and Ref. [54], three new mapping methods I_Log_GMP, XY_Log_GMP and XYI_Log_GMP are considered, which are listed in Table 2.3. As can be seen, Log_GMP outperforms I_Log_GMP, XY_Log_GMP and XYI_Log_GMP with higher recognition rates of $0.18 \%$,

Table 2.3: Recognition accuracy comparison when adding the intensity, and pixel location information to Log_GMP RCM.

\begin{tabular}{|l|l|c|}
\hline Method & $\phi(I, x, y)$ & Accuracy(\%) \\
\hline \hline Log_GMP & {$\left[\log \left(m_{v, k}(x, y)\right) p_{v, k}(x, y)\right]$} & 99.29 \\
I_Log_GMP & {$\left[I(x, y) \log \left(m_{v, k}(x, y)\right) p_{v, k}(x, y)\right]$} & 99.11 \\
XY_Log_GMP & {$\left[x y \log \left(m_{v, k}(x, y)\right) p_{v, k}(x, y)\right]$} & 99.07 \\
XYI_Log_GMP & {$\left[x y I(x, y) \log \left(m_{v, k}(x, y)\right) p_{v, k}(x, y)\right]$} & 97.29 \\
\hline
\end{tabular}


Table 2.4: Comparison of recognition rate and feature size.

\begin{tabular}{|l|c|c|}
\hline Method & Recognition rate (\%) & Feature size \\
\hline \hline Log_GMP & 99.29 & 4100 \\
G(2D $)^{2}$ PCA [48] & 95.03 & 6120 \\
GLRV [49] & 92.09 & 1680 \\
GRCM [53] & 96.74 & 1380 \\
EGRCM [54] & 97.50 & 2780 \\
PalmCode [12] & 99.50 & Two bit-planes size of $128 \times 128$ \\
CompCode [37] & 99.77 & Three bit-planes size of $128 \times 128$ \\
\hline
\end{tabular}

$0.22 \%$, and $2.00 \%$ respectively. It is denoted that adding features to feature vector $\phi_{L_{\text {og_GMP }}}$ has a negative effect on recognition performance for Log_GMP based RCM method.

\subsubsection{Recognition performance comparison}

In the second experiment, we compare Log_GMP based RCM method ( $i$. e. our proposed method) with several recently proposed state-of-arts Gabor based methods, including G(2D) ${ }^{2}$ PCA [48], GLRV [49], GRCM [53], EGRCM [54], PalmCode [12] and CompCode [37]. G(2D) ${ }^{2}$ PCA, GLRV and GRCM methods only employ the GM information. While EGRCM method constructs two group of RCMs as palmprint descriptor using GM and GP information separately, which are different from our proposed Log_GMP based RCM method. PalmCode and CompCode are two typical coding based methods, which are also based on Gabor filtering. For fair comparison, all the evaluated methods here utilize the same 20 Gabor filter functions, with the parameters set as Eq. (2.8) formulating. The recognition rates of these evaluated methods are tabulated in Table 2.4, together with the corresponding feature size. Table 2.5 lists the comparisons of execution times including feature extraction per image and similarity computation between two samples. As can be seen from Tables 2.4 and 2.5, our proposed method outperforms $\mathrm{G}(2 \mathrm{D})^{2} \mathrm{PCA}$, GLRV, GRCM, and EGRCM with higher recognition accuracies of $4.26 \%, 7.20 \%$, $4.10 \%$, and $2.98 \%$ respectively, while the feature size, and execution time are comparable. PalmCode and CompCode achieve a little higher recognition rate than our proposed Log_GMP based RCM method. However, they cost too much time for similarity computation, which limits their application in palmprint recognition. 
Table 2.5: Comparisons of execution times including feature extraction per image and similarity computation between two samples.

\begin{tabular}{|l|c|c|}
\hline Method & Feature extraction (ms) & Similarity computation (ms) \\
\hline \hline Log_GMP & 235 & 7.1 \\
G(2D) ${ }^{2}$ PCA [48] & 143 & 2.2 \\
GLRV [49] & 230 & 1.5 \\
GRCM [53] & 163 & 2.2 \\
EGRCM [54] & 240 & 4.2 \\
PalmCode [12] & 5.4 & 14.5 \\
CompCode [37] & 19.5 & 17.3 \\
\hline
\end{tabular}

\subsubsection{Conclusion and future work}

Gabor-based RCM has been introduced previously in face and palmprint recognition. In this paper, we focus on constructing a more effective feature mapping function $\phi(I, x, y)$, which is crucial to the recognition performance of RCMs based framework. The main contributions of this paper are as follows: (a) Different from the conventional Gabor magnitude (GM) based methods that take directly the GM as image features, we apply the logarithmic transformation of GM, based on the fact that GM features of each palmprint image approximate a lognormal distribution, which results in a more discriminative representation for palmprint images; (b) Different from EGRCM [54] that constructs two group of RCMs as palmprint descriptors by employing the GM and GP information respectively, we concatenate the logarithmic transformation of GM and GP to one sequence ( $i$. e. Log_GMP) as the mapping vector of RCM, which strongly enhances the discriminative ability of the corresponding RCMs due to the contribution of the correlations information of LogGM and GP; (c) The experimental results also demonstrate that adding pixel locations $(x, y)$ and intensity component $I(x, y)$ to feature vector $\phi(I, x, y)$ has a negative effect on palmprint recognition performance for our proposed Log_GMP based RCM method.

Though RCM is an effective feature extraction framework, how to introduce class-specific information is an open issue. The covariance matrices lie on a connected Riemannian manifold, the matching between two RCMs based on the used similarity measurement is time consuming. In terms of these disadvantages, we propose another algorithm to extract palmprint feature based on the statistical property of image region in our subsequent follow-on work (refer to Ref. [55]). 


\subsection{Chapter conclusion}

In this chapter, a texture presentation based on the RCM framework for palmprint recognition is proposed. With regards to the research objective which we formulate in Section 1.3.3, this chapter aims to a real-valued feature representation for an unprotected recognition system. The proposed method introduces an invariant feature representation, with the advantages of being scale and illumination independent and being robust against image rotation and translation variations. By combing the log-scaled Gabor magnitude and the Gabor phase information, the created RCM achieves improved recognition accuracy with a relatively lower feature size. However, the feature template matching speed could be relatively slow, since the used similarity measurement for RCM is computational inconvenient. 


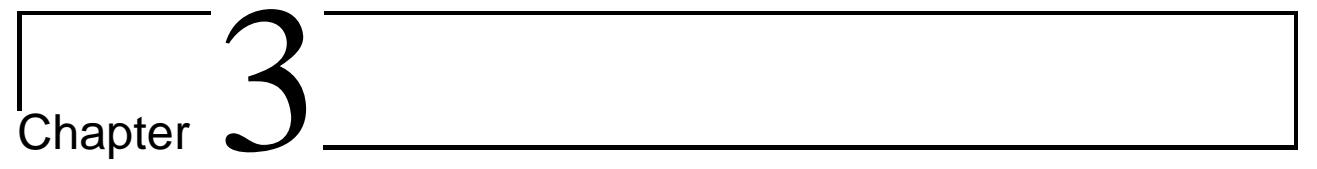

\section{Mean and standard deviation of}

\section{Gaussian}

\subsection{Chapter introduction}

PURPOSE. This chapter introduces a texture representation created by the statistics including mean and standard deviation, which is real-valued. The investigated multi-scale and multi-orientational transform is a Gabor filter bank. The proposed representation is based on the regional statistical features. In Chapter 2, a Region Covariance Matrix (RCM) representation is presented based on the assumption that the covariance of a distribution is able to discriminate it from other distributions. The feature mapping function for RCM is established based on the finding that the Gabor magnitude (GM) response is close to Lognormal distribution. In this chapter, we further study the distribution characteristic of GM response. The novel representation is proposed based on the assumption that the mean and standard deviation of a Gaussian distribution is able to discriminate it from other Gaussian distributions.

CONTENTS. In this chapter, a real-valued palmprint representation method 


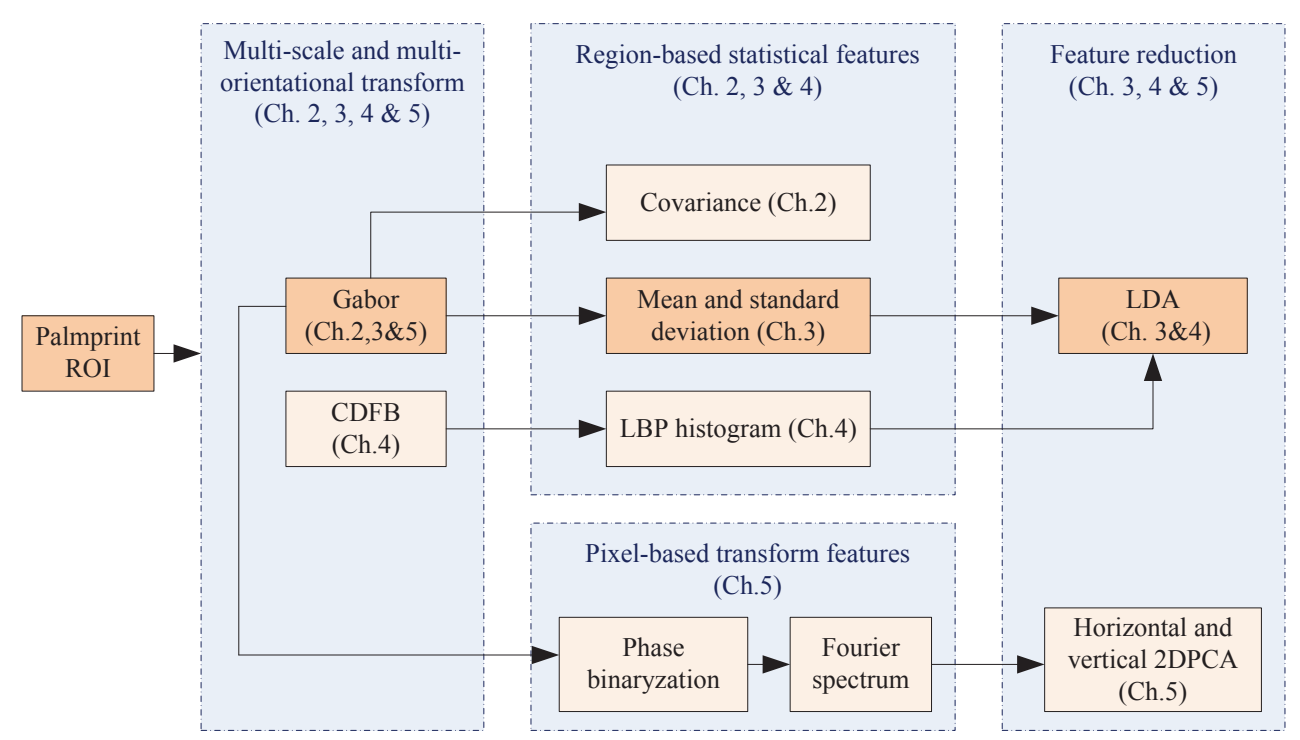

Fig. 3.1: Block diagram of our designed system, highlighting the context of Chapter 3 and its referred blocks.

is presented, which is introduced in detail in Section 3.2.3. A bank of $2 \mathrm{D}$ Gabor functions are used to filter the image firstly. The Gabor scale parameter is empirically determined. For the Gabor orientation selection, Gabor energy of an image is defined, which varies as the Gabor orientation changes. Accordingly several interesting orientations are selected for evaluation based on the characteristics of Gabor energy responses. Based on the findings that Gabor magnitude (GM) coefficient matrix of a palmprint image approximate a lognormal distribution, a Gaussian sequence can be obtained by applying the logarithmic transform on GM. Inspired by the fact that a Gaussian distribution can be represented by its mean and standard deviation without heavy information loss, we extract the mean and standard deviation values to construct the palmprint representation. For keeping the palmprint structure information, subblock partition is carried out. For recognition, several similarity measurements are evaluated. The experiments are implemented over two palmprint databases including CCD devise based images and digital scanner based images. In the context of system diagram, the content of this chapter and its referred blocks are highlighted in Figure 3.1.

PUBLICATION(s). The content of Section 3.2 of this chapter has been published in [55]. 


\subsection{Mean and standard deviation as representation}

\subsubsection{Abstract}

The 2D Gabor function has been recognized as a very useful tool in feature extraction of image, due to its optimal localization properties in both spatial and frequency domain. This paper presents a novel palmprint feature extraction method based on the statistics of decomposition coefficients of the Gabor wavelet transform. It is experimentally found that the magnitude coefficients of the Gabor wavelet transform within each subband uniformly approximate the Lognormal distribution. Based on this fact, we create the palmprint representation using two simple statistics (mean and standard deviation) as feature components after applying the logarithmic transformation of Gabor filtered magnitude coefficients for each subband with different orientation and different scale. The optimum setting of the number of Gabor filters and orientation of each Gabor filter is experimentally determined. For palmprint recognition, the popularly used Fisher Linear Discriminant (FLD) analysis is further applied on the constructed feature vectors to extract discriminative features and reduce dimensionality. All experiments are both executed over the CCD device based HongKong PolyU Palmprint Database of 7752 images and the digital scanner based BJTU_PalmprintDB (V1.0) of 3460 images. The results demonstrate the effectiveness of the proposed palmprint representation in achieving the improved recognition performance.

\subsubsection{Introduction}

Automatic personal identification has always been a hot research topic during the last decades. It has a wide range of applications such as security systems, credit card verification, scene surveillance including commercial and law enforcement application. Traditionally, automatic personal identification can be divided into two categories: token-based, such as a physical key, an ID card and a passport, and knowledge-based, such as a password. However, they can be easily spied by the criminals [8]. As an efficient and safe solution to such schemes, biometrics technology has recently been receiving wide attention from researchers, which is concerned with identifying a person by his/her physiological characteristics, such as iris, palmprint, fingerprint and face, or using some aspect of his/her behavior, such as voice, signature and 


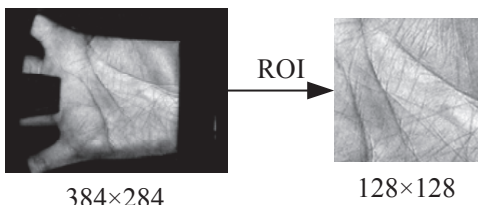

(a) HongKong PolyU

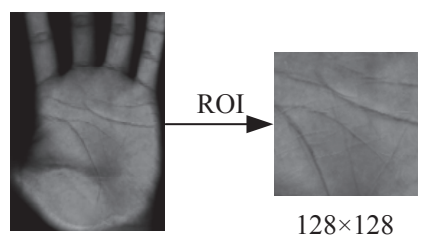

$292 \times 413$

(b) BJTU

Fig. 3.2: Typical palmprint samples and the corresponding extracted ROI images.

gesture $[17,18,21,22,24,25,30]$. By comparison, palmprint has merits such as robustness, user-friendliness, high accuracy, and cost-effectiveness. Since Zhang and Shu [33] first propose to use palmprint as a characteristic for automatic personal identification, there have been many approaches proposed for palmprint recognition (including verification and identification). Early studies focus on palmprint structure features, such as three principal palm lines, the ridge and valley structures like those in fingerprint, for off-line palmprint images of high resolution (up to $500 \mathrm{dpi}$ ) [32,33]. For online low-resolution palmprint images (less than $100 \mathrm{dpi}$ ), extracting structural features becomes much more difficult, and the mere principal palm lines do not contribute adequately to high accuracy. Figure 3.2(a) shows a typical palmprint image in the HongKong PolyU Palmprint Database and the corresponding cropped region of interest (ROI) image, captured by a CCD-based device at 75 dpi. Figure 3.2(b) shows one sample in the Beijing Jiaotong University Palmprint Database (BJTU_PalmprintDB (V1.0)), captured by a digital scanner at 72 dpi. For more information about the ROI extraction, please refer to Refs. [12] and [15]. How to construct an efficient palmprint representation from lowresolution palmprint image is still an open issue for palmprint recognition.

The recent existing algorithms for online palmprint recognition can be generally classified into three main approaches:

(1) Coding based methods, which encodes the response of a bank of filters, is an active area, since the codes are robust and can provide enough discriminative information, as well as the coding rules are usually flexible and simple. Zhang and Kong et al. [8] proposed PalmCode, which encodes the phase of the filter responses into bitwise features. Subsequently, Kong et al. [36] used a fusion rule at feature layer to further improve PalmCode. And this approach was named as FusionCode. Wu et al. [42] proposed a DoG code method which first convolves the image using 2D Gaussian filter and then encodes the zero-crossing information of horizontal and vertical gradient values, 
respectively. The schemes coding the orientation of palm lines are also popular since its stability of encoded orientation information such as competitive code (CompCode) [37] and its improved editions [40,41], palmprint orientation code (POC) [38], robust line orientation code (RLOC) [39], and so on. However, coding based methods require the precise alignment processing of codes on pairs of pixel positions ideally for palmprint matching. Though they can offset this deficiency by constructing an enlarged training set, it is highly time-consuming to determine the identity of one test palmprint, especially in a large registered database;

(2) Subspace learning based methods, such as principal component analysis (PCA) [34], Fisher linear discriminant (FLD) [35] analysis, locality preserving projection (LPP) [56], treat palmprint image as a 2D matrix. In general, these methods suffer from image variations in illumination, rotation and translation if the subspace learning is processed directly on the original intensity images. To be robust against illumination perturbations, translation and rotation after the image preprocessing, multi-resolution analysis is widely adopted by using wavelets or Gabor functions. Then the subspace learning is performed on the multi-scale or multi-orientation subband coefficients. These methods have been reported with good performance in many publications $[48,50]$. However, the subband coefficients from different scales and orientations decomposition usually generate an overwhelming high-dimensional feature space. In order to reduce the feature dimensionality, the widely used strategy is to down-sample the filtered images with a factor. However, this process might lead to a heavy loss of the discriminative information. In addition, the feature distribution of a filtered image could be far from Gaussian. Consequently, the classical dimension-reduced techniques, such as PCA and the spectral analysis, would not be optimum for feature reduction [57];

(3) Texture energy based approaches have been introduced and deemed to be among the promising methods. Li et al. [43] used four masks to highlight the distribution of line segments in horizontal, vertical and two diagonal lines, and then computed the global and local energies to represent a palmprint image. Wu et al. [44] introduced wavelet energy feature (WEF) after applying $2 \mathrm{D}$ discrete wavelet decomposition, which reflects the spatial feature of palmprint texture to some extent. However, these two methods both suffer from a limited orientation selective capability, which only distinguish information at horizontal, vertical and diagonal orientations. While there are many palmprint texture lines presenting at other orientations. More recently, to offset the global disturbance occurring on palmprint image, Pan et al. [49] divided the Gabor filtered magnitude image into two-layered partitions and then cal- 
culated the difference of standard deviation between each lower-layer subblock and its resided upper-layer block (called local relative variance (LIF)). However, the distribution of Gabor magnitude coefficients is not close to Gaussian, such standard deviation based feature representation lead to information loss. Besides, there are also feature fusion based approaches which have been reported with good performances in many publications $[58,59]$.

In this paper, each palmprint sample is considered as a texture image. To develop an efficient algorithm for the palmprint texture classification, it is essential to extract a set of texture features with powerful discriminative ability. So far, texture classification techniques have been highly advanced, among which the multi-resolution analysis approaches, such as the wavelet transform, prove to be the adequate tools to characterize different scales of texture efficiently. However, the palmprint texture is non-periodical, which is different from the regular textures like barks, bricks etc. . It contains lines of more orientations. The wavelet transform lacks in decomposing palmprint image into the arbitrary orientations. Another powerful tool of characterizing texture image is the Gabor filter bank, which provides the optimal localization of image details in a joint spatial and frequency domain. By calculating the mean and variance of the Gabor filtered image, Arivazhagan et al. [60] constructed the rotation invariant features for texture classification. Rotation normalization is achieved by the circular shift of the feature elements, assuming the texture image has a dominant direction. Subsequently, Han et al. [61] proposed the rotationinvariant and scale-invariant Gabor features, which exploited the mean and standard deviation statistics to construct the feature vector. Inspired by the high performances of these works, we investigate the simple statistics (mean and standard deviation) based on the Gabor filtering responses for constructing the palmprint representation. It is known that the representation by the mean and the standard deviation information would lead to information loss, if the distribution of one palmprint Gabor filter response is far from Gaussian. In this paper, we experimentally find that the Gabor magnitudes of one palmprint image within each subband of different scales and orientations uniformly approximate a Lognormal distribution, $i$. e. we can obtain a group of Gaussian distributions by implementing the logarithmic transformation on the Gabor filtered magnitude coefficients of each subband. Based on the simple statistics (mean and standard deviation) of these Gaussian distributions, a novel palmprint representation is constructed for palmprint recognition in this paper, the block diagram of which is shown in Fig. 3.3.

The palmprint image is firstly convolved with a bank of Gabor filters. The optimum number and the orientation parameter of filters are experimentally 


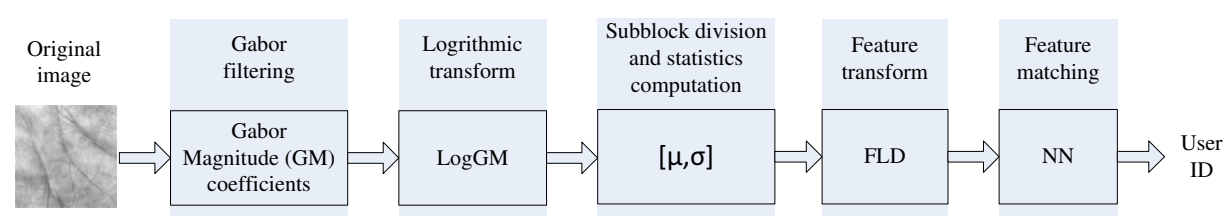

Fig. 3.3: The overall architecture of our proposed palmprint recognition system.

determined. Then the logarithmic transform is carried out on the Gabor magnitude (GM) coefficients. The resulting feature vector is denoted as LogGM. In order to preserve the spatial information, the filtered images are partitioned into some subblocks in hierarchical scales. For each subblock the Gabor magnitude distributions are nearly consistent with that in the whole image. Then, the mean and the standard derivation of LogGM features from all blocks are concatenated into a vector as palmprint representation, which turns out to be more robust against image variations and has the merit of less computational complexity. In order to enhance the discriminative ability, facilitate the realtime computation of similarity measurement and reduce the storage requirement, FLD analysis is further applied over the constructed vector. The proposed method is evaluated over the HongKong PolyU palmprint database and Beijing Jiaotong University palmprint database (BJTU_PalmprintDB (V1.0)).

The paper is organized as follows. In Sec. 3.2.3, the proposed palmprint recognition method is introduced in detail including three subsections. First, the 2D Gabor filter bank will be reviewed in Sec. 3.2.3.1. In Sec. 3.2.3.2, the proposed palmprint feature vector construction method is described. Then, the applied palmprint recognition scheme is described in Sec. 3.2.3.3. The experimental results will be demonstrated in Sec. 3.2.4. The final part is about the conclusion.

\subsubsection{Palmprint recognition based on Gabor filtered images}

Basically, a palmprint recognition system involves four key steps: namely, palmprint sample capture, image reprocessing, feature capture extraction and template matching. A successful palmprint recognition system considerably depends on the particular selection of features to represent palmprint images. Precise and concise palmprint feature extraction is crucial and desirable for determining the final recognition accuracy and computational efficiency. This paper focuses on extracting some simple statistics of Gabor filtered images as feature representation for palmprint recognition. 


\subsubsection{2D Gabor filters}

It is well known that the visual cortex of the human visual system (HVS) can be appropriately modeled as a set of independent channels, and each channel is tuned to a particular orientation and spatial frequency. Hence, a joint spatialfrequency multi-channel representation methodology is effective in characterizing texture image features. The Gabor filter family essentially performs a multi-channel representation, which is in line with the multi-channel filtering mechanism of the HVS in perceiving visual information. Based on this fact, Gabor representation can provide the optimal localization of image details in a joint spatial and frequency domain [62].

The generally used 2D Gabor function for palmprint feature extraction is formalized as following:

$$
\begin{aligned}
g_{m, n}(x, y)= & \frac{1}{2 \pi \sigma^{2}} \exp \left\{\frac{-\left(x^{2}+y^{2}\right)}{2 \sigma^{2}}\right\} \\
& \times \exp \left\{2 \pi i\left(u_{m} x \cos \theta_{n}+u_{m} y \sin \theta_{n}\right)\right\},
\end{aligned}
$$

where $i=\sqrt{-1}, u_{m}$ is the frequency of the sinusoidal wave along the direction $\theta_{n}$ from the x-axis, and $\sigma$ specifies the Gaussian envelope along $x$ and $y$ axes, which determines the bandwidth of the Gabor filter. Suppose that parameter $S$ is the total number of scales, and parameter $K$ is the total number of orientation, then $m=0,1, \ldots, S-1, n=0,1, \ldots, K-1$. In practice, a Gabor function $g_{m, n}(x, y)$ with a special set of parameters $\left(u_{m}, \theta_{n}, \sigma\right)$, is transformed into a discrete Gabor filter. In order to be robust against illumination variations, the Gabor filter needs to be zero DC (direct current), which can be formalized as:

$$
\tilde{g}_{m, n}(x, y)=g_{m, n}(x, y)-\frac{\sum_{i=-N}^{N} \sum_{j=-N}^{N} g_{m, n}(i, j)}{(2 N+1)^{2}}
$$

where $(2 N+1)^{2}$ is the size of the filter. Following the experimental results of Kong et al. [12], $\sigma$ is set to 5.6179 , which is equivalent to $N=5$.

Given an image $I(x, y)$ of size $W \times H$, its Gabor-filtered outputs are defined as:

$$
J_{m, n}(x, y)=\sum_{x_{1}} \sum_{y_{1}} I\left(x_{1}, y_{1}\right) \tilde{g}_{m, n}\left(x-x_{1}, y-y_{1}\right) .
$$


$J_{m, n}(x, y)$ is complex numbers. The Gabor magnitude (GM) responses can be denoted as

$$
G M_{m, n}(x, y)=\left\|J_{m, n}(x, y)\right\|
$$

where $\|\bullet\|$ denotes the modulus operator of a complex number.

\subsubsection{Palmprint feature vector construction}

For a given application where the Gabor based feature are used, it is essential to select the suitable Gabor parameter. In general, they are empirically determined. For instance, the original CompCode scheme [37] takes $\theta_{n}=\pi n / 6$, $n=\{0,1, \ldots, 5\}$ as the orientation of Gabor filters for feature extraction. Subsequently, Yue et al. [40] improved CompCode method by implementing a modified fuzzy C-means cluster algorithm to determine the orientation of each Gabor filter based on the statistical orientation distribution. Besides, for palmprint recognition Pan et al. [49] exploited a bank of Gabor filters with five scales $u_{m}=0.2592 / \sqrt{2^{m}}, m=\{0,1, \ldots, 4\}$ and four orientations $\theta_{n}=\pi n / 4$, $n=\{0,1, \ldots, 3\}$ for the proposed local invariant feature extraction method.

In this paper, five scales are fixed as those in Ref. [49], $u_{m}=0.2592 / \sqrt{2^{m}}$, $m=\{0,1, \ldots, 4\}$. Aiming to determine the optimum orientation parameter $\theta_{n}$, a definition of Gabor energy, denoted as $E$, is given as following:

$$
E=\sum_{x} \sum_{y} G M(x, y)
$$

Basically, palmprint texture displays as negative lines. For one Gabor filtered image at certain orientation, the smaller $E$ presents the more lines in the original intensity image. To further investigate the orientation information of the whole palmprint, four different images (two images are from the left hands, and the other ones from the right hands) in the Hong Kong PolyU Palmprint Database, are randomly selected to convolve with 180 Gabor filters at orientation $\theta_{n}=\left\{0^{\circ}, 1^{\circ}, 2^{\circ}, \ldots, 179^{\circ}\right\}$ respectively, given $m=2, u_{m}=$ $0.2592 / \sqrt{2^{2}}=0.1296$. Then the corresponding Gabor energy $E$ is calculated for each resulted Gabor mapping. Figure 3.4 shows the plots of Gabor energy versus filter orientation for all the four images. The same processes are all conducted over the BJTU_PalmprintDB (V1.0), with the corresponding plots shown in Fig. 3.5. 


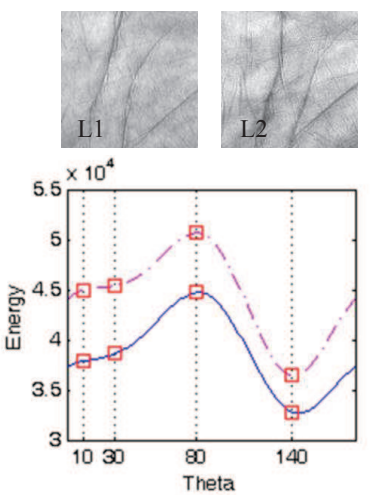

(a) Left hands
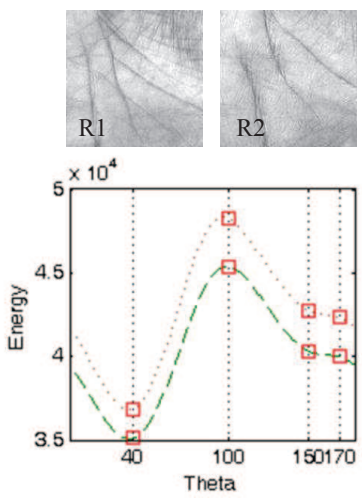

(b) Rright hands

(a) The plots of images L1 and L2 cap- (b) The plots of images R1 and R2 captured from the left hands. tured from the right hands.

Fig. 3.4: The plots of Gabor energy (Energy) vs. filter orientation (Theta) for palmprint images in the the HongKong PolyU Palmprint Database.

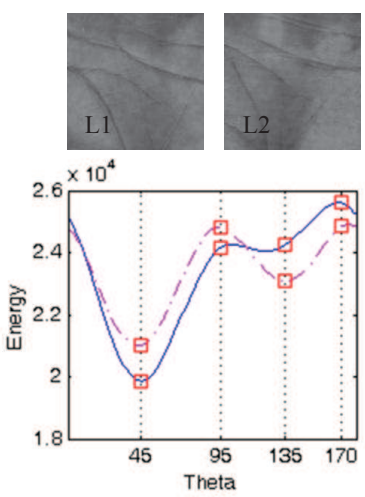

(a) Left hands
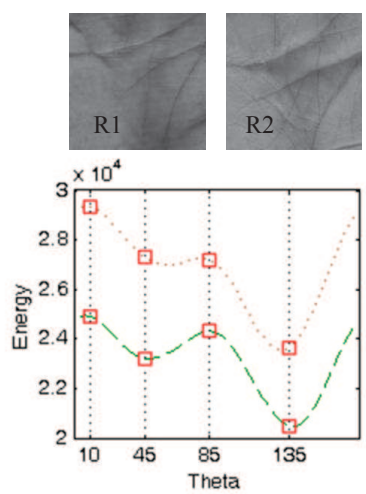

(b) Rright hands

(a) The plots of images L1 and L2 cap- (b) The plots of images R1 and R2 captured from the left hands. tured from the right hands.

Fig. 3.5: The plots of Gabor energy (Energy) vs. filter orientation (Theta) for palmprint images in the the BJTU_PalmprintDB (V1.0).

Table 3.1: The investigated orientation sets for determining the optimal Gabor filter bank.

\begin{tabular}{|c|c|}
\hline & Orientation sets \\
\hline \hline Set_1 & $\left\{45^{\circ}, 135^{\circ}\right\}$ \\
Set_2 & $\left\{0^{\circ}, 45^{\circ}, 90^{\circ}, 135^{\circ}\right\}$ \\
Set_3 & $\left\{0^{\circ}, 30^{\circ}, 60^{\circ}, 90^{\circ}, 120^{\circ}, 150^{\circ}\right\}$ \\
Set_4 & $\left\{0^{\circ}, 30^{\circ}, 45^{\circ}, 60^{\circ}, 90^{\circ}, 120^{\circ}, 135^{\circ}, 150^{\circ}\right\}$ \\
Set_5 & $\left\{10^{\circ}, 30^{\circ}, 45^{\circ}, 60^{\circ}, 85^{\circ}, 95^{\circ}, 120^{\circ}, 135^{\circ}, 150^{\circ}, 170^{\circ}\right\}$ \\
\hline
\end{tabular}


There are three distinctive characteristics which can be observed from Figs. 3.4 and 3.5. Firstly, the plots of Gabor energy distribution versus orientation are approximately uniform for different palmprints from the hands of one same side (left or right). Second, the plots for two different images from the left and right hands respectively approximate bilateral symmetry. Thirdly, there are several observable peaks and valleys in each plot. For example, there are three peaks around $\left\{10^{\circ}, 80^{\circ}, 170^{\circ}\right\}$, and three valleys around $\left\{30^{\circ}, 140^{\circ}, 150^{\circ}\right\}$ in the plots of images from the Hongkong PolyU palmprint database. While in the plots of ones from the BJTU_PalmprintDB (V1.0), the peaks and valleys appear around $\left\{10^{\circ}, 85^{\circ}, 95^{\circ}, 170^{\circ}\right\}$ and $\left\{45^{\circ}, 135^{\circ}\right\}$ respectively. Accordingly, the images from different palms can be filtered by a bank of Gabor functions at the same set of orientations. However, which and how many orientations will be optimal for portraying palmprint texture characteristics is still a question. In this work, five sets of orientations listed in Table 3.1 are investigated. The optimal Gabor filter setting is determined following the experimental results illustrated in Sec. 3.2.4.

Most previous works use Gabor magnitudes (GMs) directly for image feature extraction. In this paper, the statistical characteristics of GMs are explored for constructing palmprint representation. Given the Gabor filter parameters $\sigma=5.6179$ and $u_{m}=0.1296$, for a palmprint image from HongKong PolyU palmprint database as it displays in Fig. 3.6(a), two GMs are obtained at Gabor orientation of $\theta_{n}=45^{\circ}$ and $\theta_{n}=135^{\circ}$ respectively. Their empirical histograms resulted from the maximum likelihood estimation (MLE) algorithm, together with the fitted Lognormal densities are plotted in Figs. 3.6(b) and 3.6(d) respectively. As can be seen from them, the Lognormal densities fit the GMs very well. By carrying out the logarithmic transform, two LogGMs are generated, whose histograms are plotted in Figs. 3.6(c) and 3.6(e). As they show, the Gaussian density is well fitted to LogGM. Thus, we can conclude that each LogGM is close to a Gaussian distribution. The same fact can be found by the palmprint images from the BJTU Palmprint database. Figure 3.7(b-e) plot the corresponding histograms from a palmprint displaying in Fig. 3.7(a).

In general, a Gaussian sequence can be represented by its mean and standard deviation without heavy information loss. Accordingly, the mean and standard deviation of LogGMs from filtered images are used to construct the feature 

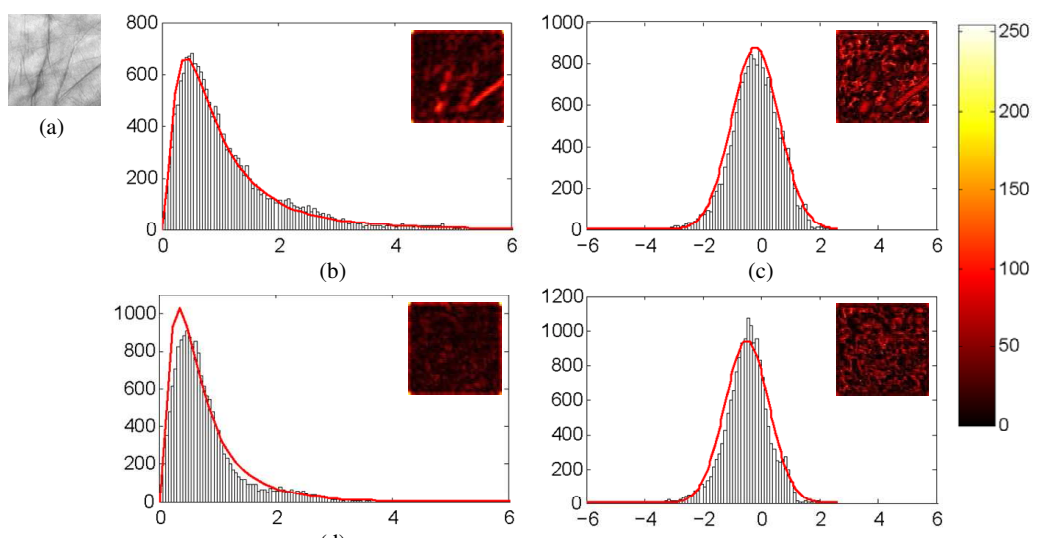

(d)

(e)

Fig. 3.6: The Lognormal distributions fitted to the empirical histograms of Gabor magnitude (GM), and the Gaussian distributions fitted to the empirical histograms of the logarithmic transformation of Gabor magnitude (LogGM) at two particular filtered subbands for an image from the HongKong PolyU Palmprint Database. (a) The image sample; (b) GM of (a) at $\sigma=5.6179, u_{m}=0.1296$, and $\theta_{n}=45^{\circ}$; (c) logarithmic transformation of (b); (d) GM of (a) at $\sigma=5.6179, u_{m}=0.1296$, and $\theta_{n}=135^{\circ}$; (e) logarithmic transformation of (d).

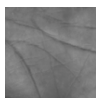

(a)

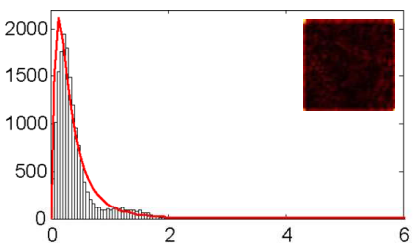

(b)

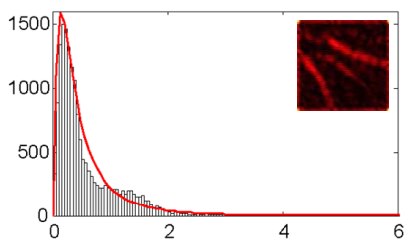

(d)

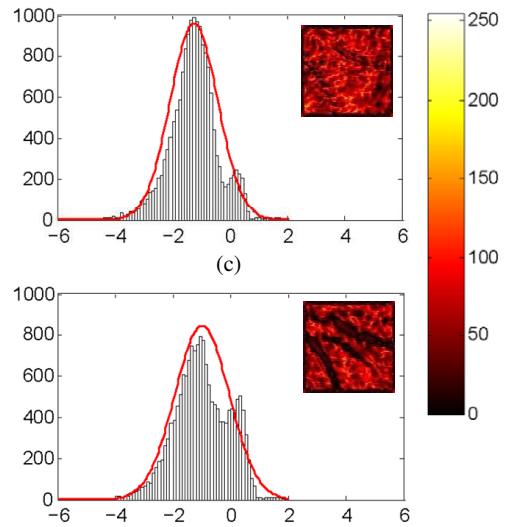

(e)

Fig. 3.7: The Lognormal distributions fitted to the empirical histograms of Gabor magnitude (GM), and the Gaussian distributions fitted to the empirical histograms of the logarithmic transform of GM (LogGM) from two filtered subbands. (a) An image from the BJTU_PalmprintDB (V1.0); (b) GM of (a) at $\sigma=5.6179, u_{m}=0.1296$, and $\theta_{n}=45^{\circ}$; (c) logarithmic transform of (b); (d) GM of (a) at $\sigma=5.6179, u_{m}=0.1296$, and $\theta_{n}=135^{\circ}$; (e) logarithmic transform of (d). 


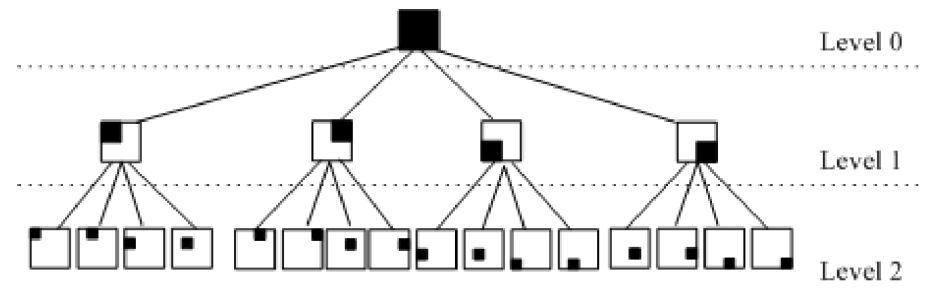

Fig. 3.8: The quadtree-based partition scheme at three levels $L=0,1,2$.

vector in this work, which can be formalized as:

$$
\begin{gathered}
\mu_{m, n}=\frac{1}{W \times H} \sum_{x} \sum_{y} \log G M_{m, n}(x, y), \\
\sigma_{m, n}=\sqrt{\frac{1}{W \times H} \sum_{x} \sum_{y}\left(\log G M_{m, n}(x, y)-\mu_{m, n}\right)^{2}}
\end{gathered}
$$

and

$$
\log G M_{m, n}(x, y)=\log \left(G M_{m, n}(x, y)\right)
$$

where $W \times H$ is the total number of the image pixels. Assuming that the scale and orientation numbers of the used Gabor filters are $S$ and $T$ respectively, the feature vector can be formulated as

$$
\left[\mu_{m, n}, \sigma_{m, n}\right] \quad m=0,1, \ldots, S-1 ; n=0,1, \ldots, T-1 .
$$

Furthermore, in order to keep the palmprint spatial structure information, we divide the original image into some subblocks using quadtree-based partition scheme. Figure 3.8 illustrates a sketch map of the quadtree with two-level partitions. The distributions of these obtained subblocks are investigated. The plots of several generated subblocks from one image are shown in Fig. 3.9, together with the histograms of corresponding GMs and LogGMs with the fitted distributions. As can be seen from it, the LogGM from local subblock is also close to Gaussian distribution. Thus, the mean and standard deviation from the subblocks are extracted. Given the quadtree level $L$, the number of subblocks is $K=4^{1}+4^{2}+\ldots+4^{L}$. Finally, all the mean and standard deviation values are concatenated into one sequence as the palmprint representation, 

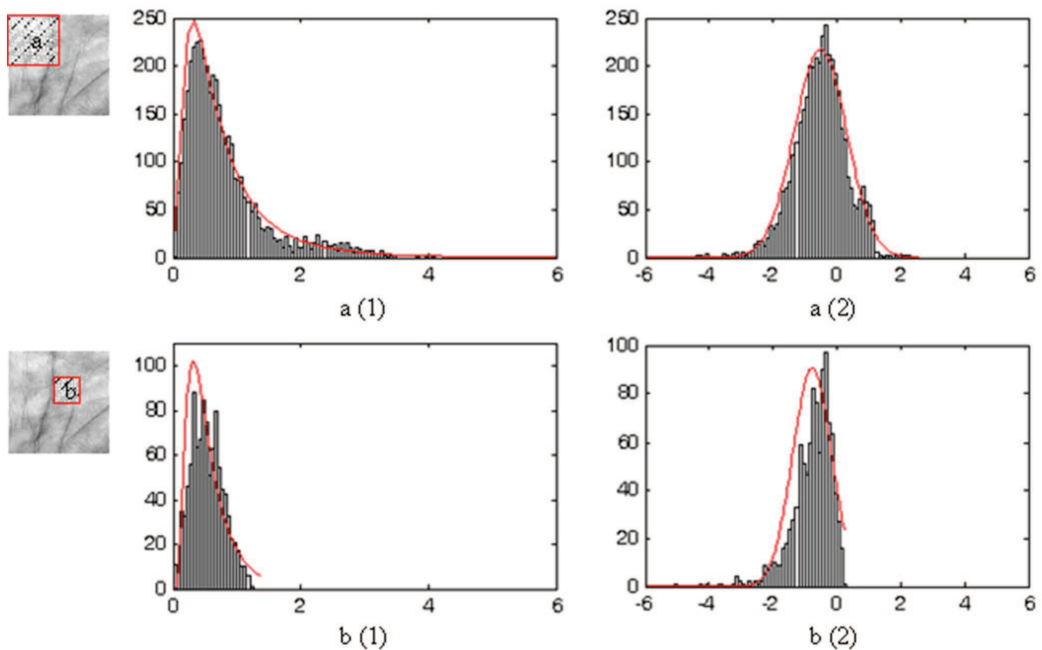

Fig. 3.9: The histogram plots with their fitted distributions of Gabor magnitude (GM) and corresponding Logarithmic transform of GM (LogGM) from two subblocks. 'a' presents a subblock at the quadtree level $L=1$, and 'b' displays a subblock at $L=2$; (1) denotes GM plot and (2) shows LogGM plot.

formulated by:

$$
\left[\mu_{m, n, k}, \sigma_{m, n, k}\right] \quad m=0,1, \ldots, S-1 ; n=0,1, \ldots, T-1 ; k=0,1, \ldots, K
$$

where $k=0$ corresponds to the statistics from the whole filtered image. The optimum partition level $L$ for our proposed approach will be determined based on the experimental results illustrated in the next section.

\subsubsection{Palmprint recognition}

In general, when two images $i$ and $j$ are respectively represented by a feature vector formed by the mean and standard deviation values, the similarity distance $d_{m, n}(i, j)$ between them can be computed as follows:

$$
d_{m, n}(i, j)=\left|\frac{\mu_{m, n}^{(i)}-\mu_{m, n}^{(j)}}{\alpha\left(\mu_{m, n}\right)}\right|+\left|\frac{\sigma_{m, n}^{(i)}-\sigma_{m, n}^{(j)}}{\alpha\left(\sigma_{m, n}\right)}\right|
$$


or

$$
d_{m, n}(i, j)=\sqrt{\left(\mu_{m, n}^{(i)}-\mu_{m, n}^{(j)}\right)^{2}+\left(\sigma_{m, n}^{(i)}-\sigma_{m, n}^{(j)}\right)^{2}},
$$

where $\alpha\left(\mu_{m, n}\right)$ and $\alpha\left(\sigma_{m, n}\right)$ are the standard deviations of feature component $\mu_{m, n}$ and $\sigma_{m, n}$ over the entire database. Here they are used for feature normalization.

The similarity measurements mentioned above have been reported to be efficient for texture classification [60,61]. However, they are both in an unsupervised manner. In this work, Fisher Linear Discriminant (FLD) analysis [35] is applied on the constructed feature vectors, which is more suitable for a classification problem in the supervised manner. It is to extract more discriminative features, as well as to reduce feature dimensionality. Finally, the nearest neighbor classifier is used for palmprint recognition, which contributes to the computational efficiency.

\subsubsection{Experimental results}

In this section, we evaluate the performance of proposed approach over two databases: (1) Hong Kong Polytechnic University (PolyU) palmprint database, and (2) Beijing Jiaotong University palmprint database (BJTU_PalmprintDB $(\mathrm{V} 1.0))$. In the first part of this section, we briefly introduce these two databases. Following sets of experiments are carried out in the next part of this section.

I. Validating which set in Table 3.1 (the Gabor orientation parameter setting) and how many quadtree levels (the image partition setting) can be optimum for portraying palmprint texture characteristics.

II. Verifying the contribution of logarithmic transform on the Gabor magnitude responses (GMs).

III. Testing the performances of three similarity measurements mentioned above.

IV. Comparing the recognition performance of our proposed approach with several state-of-the-art palmprint recognition methods. 


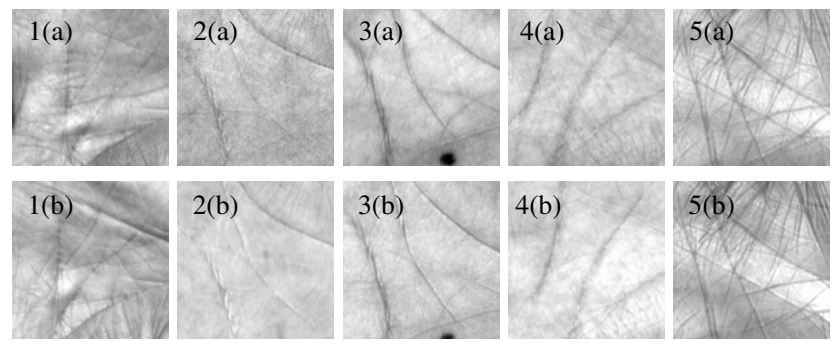

Fig. 3.10: Five typical palmprint images from different palms in the HongKong PolyU Palmprint Database. (a) and (b) denote the images captured from a same palm in the first and second session respectively.
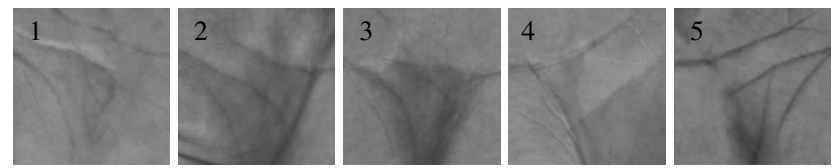

Fig. 3.11: Five typical palmprint images from different palms in the BJTU_PalmprintDB (V1.0).

\subsubsection{Palmprint Databases}

The HongKong Polytechnic University (PolyU) Palmprint Database, which has become the de facto standard for evaluating the palmprint recognition technologies, contains 7752 grayscale images in BMP image format corresponding to 386 different palms. The palmprint images captured by CCD-based device are collected in two sessions. Each time, the subject is asked to provide around ten images from the left palm and ten images from the right palm. The average interval between the two sessions is two months. The resolution of original images is $384 \times 284$ pixels at 75 dpi. In our experiments, by performing the similar preprocessing approach described in Ref. [12], palmprint is orientated and the region of interest (ROI) of size $128 \times 128$ is cropped. Figure 3.10 displays five cropped images from five typical palmprint images in this database. Figure 3.10 (a) and (b) denote the samples of the same palm collected in the first and second session respectively. As it shows, the illumination condition is different.

The Beijing Jiaotong University Palmprint Database (BJTU_PalmprintDB (V1.0)) contains 3460 grayscale images in BMP image format corresponding to 346 palms. Different from the samples in the HongKong PolyU Palmprint Database, they are captured by a Fujitsu fi-60F flat-panel scanner of high speed. The image is size of $292 \times 413$ at $72 \mathrm{dpi}$. The subjects providing palm- 
print images include 173 volunteers mainly from students and staff at Beijing Jiaotong University. Ten images are captured from the left and right palms respectively. By performing the similar preprocessing approach described in Ref. [15], the ROI size of $128 \times 128$ is cropped. The cropped images from five typical palms in this database are shown in Fig. 3.11. As it shows, the illumination condition varies in one image, which is because of the uneven structure of palm. In addition, there are some lines of wrinkled shape, which are resulted from the asymmetric press when the palm touches the flat-panel scanner.

\subsubsection{Gabor orientation parameter and image partition settings}

Image representation based on Gabor filtering has turned out to be a good fitting to the receptive field profiles ( $i$. e. , the impulse responses of the cells) in the striate cortex, so that it provides the optimal localization of image details in a joint spatial and frequency domain [45]. The Gabor filter parameters are usually application-depended. In this work, five scales of the sinusoidal wave frequency are considered the same as those in Ref. [49]. The parameter $\sigma$ specifying the Gaussian envelope is set to 5.6179 following the experiment results in Ref. [12]. Regarding to the plot characteristics shown in Figs. 3.4 and 3.5, a group of orientation sets tabulated in Table 3.1 are investigated for evaluating recognition performance of our proposed method. In addition, the setting of quadtree partition level also has a considerable impact on the recognition performance. A large subblock may result in the loss of spatial structure information, while a small subblock might lead to noise sensitiveness and computation burden. In this experiments, the quadtree levels range from 1 to 3 are investigated.

Three images of each class are randomly selected for training and the remaining ones for test. Thus, for HongKong PolyU palmprint database the training set and test set contain 1158 and 6594 images respectively. And for BJTU_PalmprintDB (V1.0) the training set and test set contain 1038 and 2422 respectively. For evaluation, ten runs of such a partition are executed. The average, top and bottom recognition rates by each evaluated parameter setting over ten runs are listed respectively. The experimental results on the HongKong PolyU palmprint database are tabulated in Table 3.2, and those on the BJTU_PalmprintDB (V1.0) are tabulated in Table 3.3. Besides, the average recognition rates over ten runs are plotted in Fig. 3.12, which differ as the Gabor orientation set and quadtree-based partition level vary. 
Table 3.2: The mean, top and bottom recognition rates over ten-run executions on the HongKong PolyU database when the Gabor orientation sets and quadtree-based partition level vary.

\begin{tabular}{|c|c|c|c|c|c|}
\hline & Set_1 & Set_2 & Set_3 & Set_4 & Set_5 \\
\hline \hline$L=1$ & & & & & \\
Mean & 95.25 & 98.10 & 99.04 & 99.04 & 99.06 \\
Top & 95.95 & 98.71 & 99.59 & 99.61 & 99.47 \\
Bottom & 94.63 & 97.33 & 98.32 & 98.42 & 98.68 \\
\hline$L=2$ & & & & & \\
Mean & 98.72 & 98.63 & 99.27 & 99.45 & 99.43 \\
Top & 98.95 & 99.29 & 99.45 & 99.74 & 99.77 \\
Bottom & 98.45 & 98.09 & 98.94 & 98.97 & 99.24 \\
\hline$L=3$ & & & & & \\
Mean & 98.13 & 99.00 & 99.10 & 99.04 & 99.19 \\
Top & 98.51 & 99.35 & 99.51 & 99.58 & 99.59 \\
Bottom & 97.76 & 98.54 & 98.65 & 98.73 & 98.74 \\
\hline
\end{tabular}
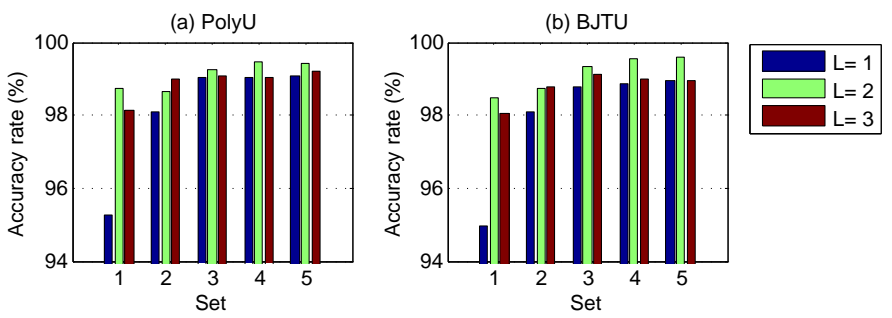

Fig. 3.12: The mean recognition rate of ten runs versus five Gabor orientation sets and three quadtree-based partition levels on the HongKong PolyU and BJTU databases respectively.

As can be seen from Tables 3.2, 3.3 and Fig. 3.12, the settings with set_4 and set_5 at $L=2$ perform significantly better than the others, which achieve 99.45\% and $99.43 \%$ recognition rates on the HongKong PolyU Palmprint Database while $99.53 \%$ and $99.59 \%$ on the BJTU_PalmprintDB (V1.0). $L=1$ and $L=3$ both perform much worse than $L=2$. This could be because $L=1$ generates too large subblocks to keep the palmprint spatial structure information, while $L=3$ results in too small subblocks to be robust against within-class variations. Set_4 and set_5 achieve approximately the same recognition performance. So we can conclude that adding two more Gabor filters based on the setting of set_4 dose not improve the recognition performance distinctively. But it enhances the computational complexity and increases the storage burden. Accordingly, for the proposed methods, the Gabor orientation set of $\left\{0^{\circ}, 30^{\circ}, 45^{\circ}, 60^{\circ}, 90^{\circ}, 120^{\circ}, 135^{\circ}, 150^{\circ}\right\}$ and the quadtree level of $L=2$ turn out to be the optimum parameter setting, which will be used in all the sequent experiments. 
Table 3.3: The mean, top and bottom recognition rates over ten-run executions on the BJTU database when the Gabor orientation set and the quadtree-based partition level vary.

\begin{tabular}{|c|c|c|c|c|c|}
\hline & Set_1 & Set_2 & Set_3 & Set_4 & Set_5 \\
\hline \hline$L=1$ & & & & & \\
Mean & 94.95 & 98.10 & 98.76 & 98.87 & 98.97 \\
Top & 95.50 & 98.64 & 99.14 & 99.17 & 99.26 \\
Bottom & 93.68 & 97.48 & 98.47 & 98.64 & 98.43 \\
\hline$L=2$ & & & & & \\
Mean & 98.48 & 98.73 & 99.35 & 99.53 & 99.59 \\
Top & 98.76 & 99.22 & 99.63 & 99.79 & 99.83 \\
Bottom & 98.14 & 98.35 & 98.97 & 99.38 & 99.46 \\
\hline$L=3$ & & & & & \\
Mean & 98.05 & 98.80 & 99.11 & 98.99 & 98.94 \\
Top & 98.35 & 99.17 & 99.46 & 99.30 & 99.42 \\
Bottom & 97.56 & 98.06 & 98.84 & 98.68 & 98.35 \\
\hline
\end{tabular}

Table 3.4: Performance comparison between GM and LogGM when the number of training samples per class varies from 2 to 5 .

\begin{tabular}{|l|c|c|c|c|c|c|c|c|c|}
\hline & \multicolumn{4}{|c|}{ PolyU } & & \multicolumn{4}{|c|}{ BJTU } \\
\cline { 8 - 10 } \cline { 7 - 9 } & 2 & 3 & 4 & 5 & & 2 & 3 & 4 & 5 \\
\hline \hline GM & & & & & & & & & \\
Mean & 98.13 & 99.36 & 99.49 & 99.75 & & 96.73 & 98.76 & 99.08 & 99.05 \\
Top & 98.61 & 99.58 & 99.76 & 99.91 & & 97.07 & 99.17 & 99.22 & 99.42 \\
Bottom & 97.55 & 99.01 & 99.29 & 99.64 & & 96.42 & 98.51 & 98.55 & 98.67 \\
\hline LogGM & & & & & & & & & \\
Mean & 99.11 & 99.55 & 99.74 & 99.81 & & 99.09 & 99.52 & 99.62 & 99.46 \\
Top & 99.41 & 99.70 & 99.98 & 99.93 & & 99.21 & 99.55 & 99.76 & 99.71 \\
Bottom & 98.75 & 99.24 & 99.31 & 99.71 & & 98.92 & 99.46 & 99.52 & 99.13 \\
\hline
\end{tabular}

\subsubsection{Effects of logarithmic transform of GMs}

As described in Sec. 3.2.3.2, our proposed palmprint representation is constructed by some simple statistics (mean and standard deviation). Instead of directly calculating the mean and standard deviation of Gabor magnitude responses (GMs), the logarithmic transform is required to be carried out ahead. In order to evaluate the effects of logarithmic transform, the performances based on GMs and LogGMs are compared. The mean, top and bottom recognition rates of five-run executions are investigated when the number of training sample per class varies from 2 to 5 . The experimental results are tabulated in Table 3.4. Figure 3.13 plots the average recognition rates versus the number of train samples per class. As can be seen, LogGMs performs better than GMs with given all the considered training set size. Especially when two samples are used for training, LogGMs achieves $99.11 \%$ and $99.09 \%$ respectively over 

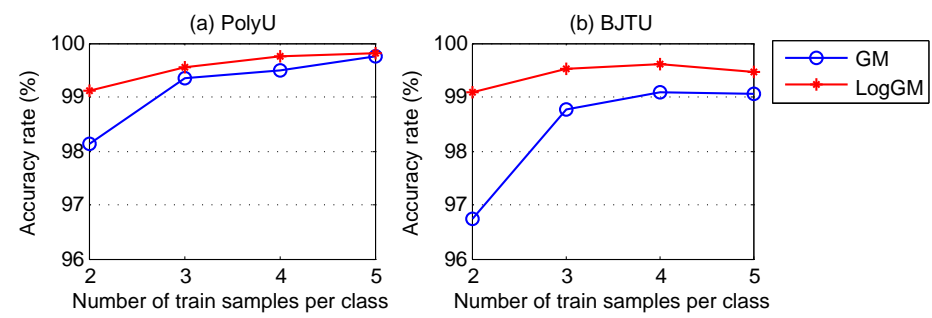

Fig. 3.13: Average recognition rate versus the number of training samples per class.

Table 3.5: Recognition rate (\%) comparison of different similarity computation methods for our proposed feature representation.

\begin{tabular}{|l|c|c|c|c|c|c|c|}
\hline & \multicolumn{3}{|c|}{ PolyU } & & \multicolumn{3}{|c|}{ BJTU } \\
\cline { 2 - 4 } \cline { 5 - 7 } & Dis_1 & Dis_2 & Ours & & Dis_1 & Dis_2 & Ours \\
\hline \hline Mean & 96.68 & 96.16 & 99.55 & & 96.76 & 95.76 & 99.52 \\
Top & 97.03 & 96.41 & 99.70 & & 97.11 & 95.95 & 99.55 \\
Bottom & 96.03 & 95.75 & 99.24 & & 96.20 & 95.62 & 99.46 \\
\hline
\end{tabular}

the HongKong PolyU Palmprint Database and BJTU_PalmprintDB (V1.0), which performs better with a large margin than GMs by $98.13 \%$ and $96.73 \%$ respectively. This could be because GMs are far from Gaussian so that the representation by the mean and standard deviation values leads to heavy loss of discriminative information. By implementing the logarithmic transform, the Gaussian sequences are approximately obtained.
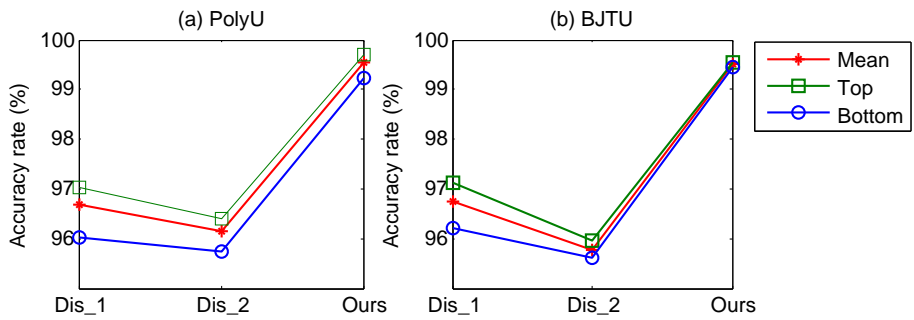

Fig. 3.14: The mean, top and bottom recognition rates over five-run executions by different similarity measurements. 


\subsubsection{Effects of different similarity measurements}

For classification based on the features formed by mean and standard deviation values, the widely used similarity measurements are formulated as Eqs. (3.11) and $(3.12)[60,61]$. In this work, FLD analysis is processed after the feature vector construction. To verify the contribution of FLD, we compared it with the other similarity measurements mentioned in this paper over the same feature vector. The methods formulated as Eqs. (3.11) and (3.12) are denoted as Dis_1 and Dis_2 respectively. Three images of each class are randomly selected to construct the training set for each execution. The mean, top and bottom recognition rates over five executions by the considered similarity measurements are investigated. The results are tabulated in Table 3.5 and plotted in Fig. 3.14. As can be seen from them, the proposed method outperforms Dis_1 and Dis_2 by achieving higher average recognition rates of $2.87 \%$ and $3.39 \%$ over the HongKong PolyU Palmprint Database, and that of $2.76 \%$ and $3.76 \%$ over the BJTU_PalmprintDB (V1.0). The performance improvement can be attributed to the excellent properties of FLD analysis, which can extract features of more discriminative ability, reduce the feature dimensionality and the computation complexity. In addition, Dis_1 performs better than Dis_2 due to the normalization process, which puts an equal emphasis on the mean and the standard deviation features.

\subsubsection{Performances of the proposed method}

In this section, the proposed method is compared with several state-of-theart methods, which are all based on the Gabor features including G_LIF [49], G_(2D) ${ }^{2}$ PCA [48] and EGRCM [54]. As they reported, G_LIF uses 20 Gabor functions with five scales and four orientations, $\mathrm{G}_{-}(2 \mathrm{D})^{2} \mathrm{PCA}$ uses 30 Gabor functions with five scales and six orientations, and EGRCM uses 40 Gabor functions with five scales and eight orientations.

In the first group of experiments, three images per palm are randomly selected to form the training set, and the remaining samples in the database are considered to be the test set. Ten random splits are executed for all these methods. The average recognition performances are listed in Table 3.6. As it shows, the proposed method achieves the highest recognition rate $(99.44 \%$ on the HongKong PolyU palmprint database and $99.53 \%$ on the BJTU_PalmprintDB (V1.0)) with the smallest feature dimensionality (385 on the HongKong PolyU palmprint database and 345 on the BJTU_PalmprintDB (V1.0)). G_LIF per- 
Table 3.6: Recognition rate (I_Rate) and feature dimension (F_Size) comparisons with several other methods. Three samples per class are randomly selected for training and the remaining ones for test. Ten random splits are executed. The average recognition performances are listed here.

\begin{tabular}{|l|c|c|c|c|c|}
\hline \multirow{2}{*}{ Method } & \multicolumn{2}{|c|}{ PolyU } & & \multicolumn{2}{c|}{ BJTU } \\
\cline { 2 - 3 } \cline { 5 - 5 } & I_Rate & F_Size & & I_Rate & F_Size \\
\hline \hline G_LIF [49] & $91.92 \%$ & 1680 & & $92.42 \%$ & 1680 \\
G_(2D) ${ }^{2}$ PCA [48] & $95.03 \%$ & 6120 & & $92.96 \%$ & 4800 \\
EGRCM [54] & $96.31 \%$ & 9460 & & $97.63 \%$ & 9460 \\
Ours & $99.44 \%$ & 385 & & $99.53 \%$ & 345 \\
\hline
\end{tabular}

Table 3.7: Recognition rate (I_Rate) comparisons with several other methods. Three samples per class from the first session are randomly selected for training and all the samples from the second session are for test. Ten random splits are executed. The average recognition performances are listed.

\begin{tabular}{|l|c|c|c|c|}
\hline Method & G_LIF [49] & G_(2D) ${ }^{2}$ PCA [48] & EGRCM [54] & Ours \\
\hline \hline I_Rate (\%) & 56.71 & 78.61 & 85.63 & 97.07 \\
\hline
\end{tabular}

forms worst, possibly because it exploits the simple standard deviation information of GMs to construct the feature representation without considering the effect of non-Gaussian distribution of GMs. G- $(2 \mathrm{D})^{2} \mathrm{PCA}$ achieves 95.03\% on the HongKong PolyU palmprint database while $92.96 \%$ on the BJTU_PalmprintDB (V1.0). The performance of G_(2D) ${ }^{2} \mathrm{PCA}$ can be affected considerably by the illumination variations. EGRCM performs significantly better than G_LIF and $G_{-}(2 \mathrm{D})^{2}$ PCA because it constructs the feature representation by both the Gabor magnitude (GM) and the Gabor phase (GP) information. However, the effect of non-Gaussian distribution is also out of consideration, which might lead to loss of discriminative information. In addition, EGRCM creates a feature space of high dimensionality 9460, which aggravates the burdens of storage and computation. It should be noticed that, in our previous work [16], a more efficient feature mapping function is proposed for palmprint representation based on the region covariance matrix (RCM), which improves the recognition performance by taking the LogGM's Gaussian characteristic into account. However, the RCM based approaches work in the unsupervised manner, which have limit in recognition performance improvement. To sum up, the proposed method can achieve high recognition rate (about $99.50 \%$ ) with smaller features (assuming all the images are from $C$ palms, the feature size will be $C-1$ ) which leads to the computational efficiency. 
The second group of experiments are executed on the HongKong PolyU palmprint database, in which the samples are collected in two sessions with different illumination conditions. Three samples per class from the first session are randomly selected for training, and all the samples from the second session are for test. So the training and test samples differ in illumination. The recognition rates are averaged over ten random splits, which are listed in Table 3.7. As it shows, the proposed method performs best. In addition, by comparing the results in Tables 3.6 and 3.7, we can find that the recognition rate can be affected considerably by the image quality.

\subsubsection{Conclusion}

In this paper, we propose a novel feature representation formed with some simple statistics (mean and standard deviation) of Gaussian models for palmprint recognition. Instead of exploiting the Gabor magnitude responses (GMs) directly as many current reported methods, we investigate the statistical distribution of GMs. It is empirically found that the GM matrix of each filtered subband approximates a Lognormal distribution. By implementing the logarithmic transform respectively on the GMs of all the Gabor filtered subbands with different scales at different orientations, we obtain a group of Gaussian sequences. Subsequently, the mean and standard deviation of each Gaussian sequence are calculated and concatenated to form the feature representation. For keeping the image spatial structure information, the quadtree-based partition is carried out for subblocks. Experimentally we get the optimal setting of involved parameters including Gabor orientation and the partition level for portraying the palmprint texture characteristics. The contribution of logarithmic transform for recognition performance improvement is verified. By comparing several popular similarity measurements, we find that the Fisher Linear Discriminant (FLD) analysis on the feature space created by the mean and standard deviation plays an important role. It contributes to the recognition rate improvement and the feature dimensionality reduction. The comparisons with the other Gabor based methods further verify the efficiency of the proposed method in recognition accuracy and computational efficiency. However, the recognition rate by the proposed method decreases when the image illumination varies, since the features are from the statistical values which are not robust to illumination changes. This issue could be addressed in our future work. 


\subsection{Chapter conclusion}

In this chapter, a real-valued palmprint representation is introduced. With regards to the research objective which we formulate in Section 1.3.3, this chapter targets to an unprotected recognition system. The mean and standard deviation of Gaussian sequences are extracted to distinguish classes so that it turned out to be robust against image translation and rotation. The recognition accuracy is depended on the Gabor orientation selection and the subblock partition. Both of the logarithmic transform on GM and the FLD analysis contribute to the recognition performance improvement. However, the recognition accuracy decreases when the illumination condition changes between the compared images. 


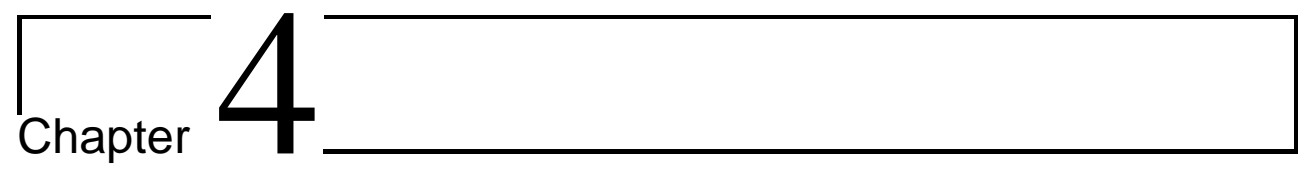

\section{Local binary pattern histogram}

\subsection{Chapter introduction}

PURPOSE. This chapter presents a real-valued palmprint representation based on the directional filter bank (CDFB) transform and the local binary pattern (LBP) operator. The CDFB transform generates a 2D decomposition of energy shiftable ( $i$. e. translation invariance) and scalable. The LBP operator, which is gray scale invariant, creates the region-based histogram statistical features. In Chapters 2 and 3, the Gabor filters are exploited for the multi-scale and multi-orientational decomposition, which provide high redundancy information. In this chapter, other multi-scale and multi-orientational transforms which can provide lower redundancy information are investigated, including the directional filter bank (CDFB) transform, dual-tree complex wavelet transform (DTCWT), discrete Contourlet transform and Nonsubsampled Contourlet transform (NSCT). Lower redundancy can lead to less storage requirement and lower computational complexity.

CONTENTS. In this chapter, the directional filter bank (CDFB) transform and the local binary pattern (LBP) operator are firstly introduced. Based on them, the proposed presentation method is presented in Section 4.2.3. The adjustable system parameters contain the scale and directional resolution of CDFB transform, and the size of LBP operator, which are determined by 


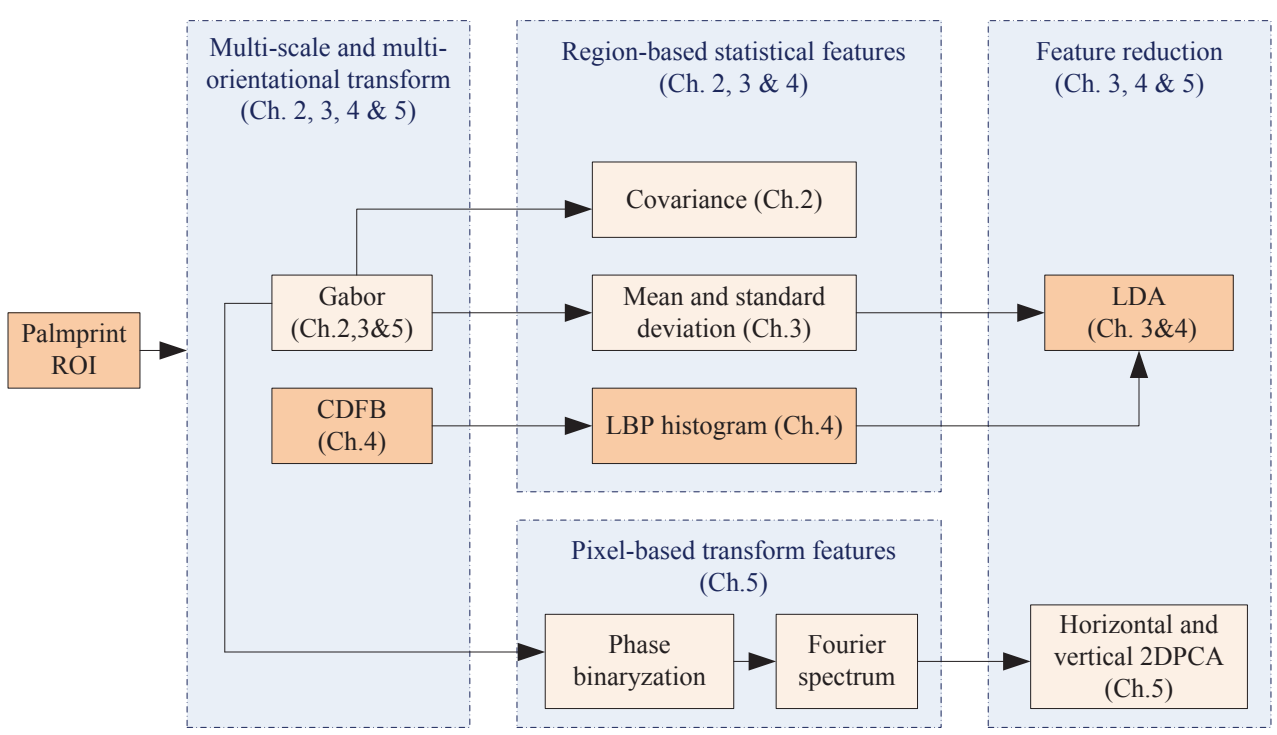

Fig. 4.1: Block diagram of our designed system, highlighting the context of Chapter 4 and its referred blocks.

the experiments presented in Section 4.2.4. For verifying the efficiency of the proposed method, other multi-scale and multi-orientational transforms are compared with the CDFB transform, and several LBP variants are compared with our used uniform LBP operator. In addition, the recently proposed sorts of local Gabor pattern operators and several Gabor-based palmprint recognition approaches are also compared with the proposed method over the HongKong PolyU palmprint database. In the context of system diagram, the content of this chapter and its referred blocks are highlighted in Figure 4.1.

PUBLICATION(s). The content of Section 4.2 of this chapter has been published in [63]. 


\subsection{Shift and gray scale invariant representation on}

\section{CDFB and LBP}

\subsubsection{Abstract}

In this paper, a novel feature extraction framework is presented for palmprint identification, which provides a shiftable and gray scale invariant description of image achieving high identification accuracy at a low computational cost. The image is firstly decomposed by the shiftable complex directional filter bank (CDFB) transform which provides a two-dimensional (2-D) decomposition of energy shiftable and scalable multiresolution, arbitrarily directional resolution, low redundant ratio, and efficient implementation. Further, the subband coefficients of CDFB decomposition are operated by the uniform local binary pattern (LBP) which is gray scale invariant and contains information about the distribution of the local micro-patterns. The resulting LBP mappings are divided into many subblocks, over which the statistical histograms are achieved independently. Finally, a Fisher linear discriminant (FLD) classifier is learned in the statistical histogram feature space for palmprint recognition. Experiments are executed over the HongKong PolyU palmprint database of 7752 images. To verify the high performance of our proposed feature descriptor, several other multiresolution and multidirectional transforms are also investigated including Gabor filter, dual-tree complex wavelet and Contourlet transforms. The experimental results demonstrate that CDFB yields the most promising performance balancing the identification accuracy, storage requirement and computational complexity for our proposed feature extraction framework.

\subsubsection{Introduction}

Palmprint-based personal recognition has become an active research topic in recent years. Compared with the other currently available biometric features, palmprints contain more distinctive information than fingerprints; palmprints acquisition devices are much cheaper than iris devices; palmprints can build a higher accurate biometrics system than face and voice [64]. In addition, palmprints can be extracted from low-resolution images. In consideration of these advantages, palmprints have been investigated extensively in automated 
personal recognition field in recent years. Among the recently reported palmprint recognition approaches, coding-based methods are deemed to be the most promising due to the strengths of small feature size and high recognition accuracy $[12,36,37,39,41,65]$. The orientation and phase information of palm lines are typically encoded as binary or integer numbers, which are robust to illumination variations. However, the coding-based methods are commonly based on pixel-to-pixel matching comparison using the Hamming distance or angular distance, which often causes poor matching results due to the unavoidable palmprint translation. To offset this deficiency, constructing an enlarged training set is required by rotating and shifting the current samples, which leads to high computational complexity. Thus the coding based methods perform well when used under the biometric verification mode, which is based on one-to-one matching operation. But for the identification system (one-tomany matching), especially with a large registered database, the coding based methods are inferior due to the low matching speed resulting from repeatedly rotating and shifting the sample feature planes. Other reported methods showing good recognition performances are mainly based on subspace analysis techniques in the intensity or transformed domain like Gabor and wavelet spaces $[56,66,67]$. However, the pixel gray intensity based subspace analysis methods typically suffer from being sensitive to the geometric transformation such as image translation and illumination variations; Gabor filtering is both memory and time intensive to extract the multiresolution and multiorientational coefficients; while 2-D discrete wavelet suffers from limited orientation selective capability, which only distinguishes information at horizontal, vertical and diagonal orientations.

Recently, extracting the local texture features as palmprint representation is another active topic. The approaches based on the local texture descriptors have been extensively studied for texture classification, face recognition and other applications in last decades [49,60,61,68-81]. Local binary pattern (LBP) [69], simple yet efficient, is a powerful texture operator, which is originally proposed for texture classification. Due to its tolerance against illumination changes and its computational simplicity, LBP has led to great success in computer vision and image processing. By investigating its working mechanism further, many variations of LBP have been reported, such as Local Ternary Pattern (LTP) [73], dominant LBP [74], center-symmetric LBP [75], LBP Variance (LBPV) [76], Local Derivative Pattern (LDP) [77], Completed LBP (CLBP) [78], and so on. On the other hand, by applying LBP analysis on the Gabor features, many local Gabor texture patterns have been proposed. Zhang et al. [70] present a local Gabor binary pattern histogram sequence (LGBPHS) to represent face, which encodes Gabor magnitude re- 


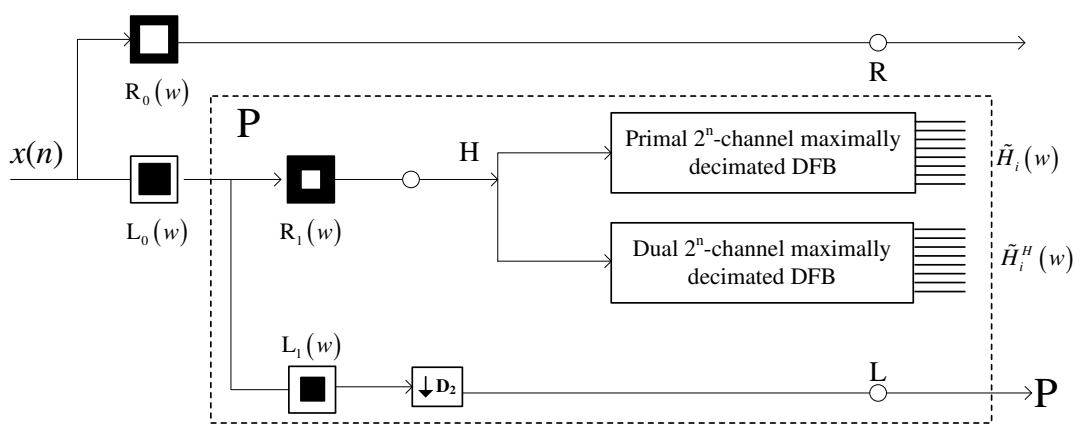

Fig. 4.2: PDTDFB structure. Blocks $\mathrm{P}$ can be reiterated at lower scale for a multiscale representation.

sponses and improves recognition performance significantly. Subsequently, Lei et al. [71] enhance LGBPHS by encoding discriminative information of face appearance not only in spatial domain, but also in Gabor frequency and orientation domains. Besides Gabor magnitude coefficients, the Gabor phase part has also been investigated intensively. W. Zhang et al. [79] study the potentials of Gabor phase when fusing with LBP for face recognition. B. Zhang et al. [80] propose two ways to encode Gabor phase variations, global Gabor phase pattern (GGPP) and local Gabor phase pattern (LGPP). Then, a kernel learning method of histogram of local Gabor phase pattern (K-HLGPP) is proposed [72]. More recently, Xie et al. [81] propose local Gabor XOR pattern (LGXP), which encodes Gabor phase by local XOR operator, and fuse it with local Gabor magnitude patterns by block-based Fisher's linear discriminant (BFLD).

Though local Gabor patterns based approaches appear to be toward high accuracy, they result in many histogram features of high dimensionality. The commonly used block partition process further increases the histogram feature size in multiples. For reducing feature dimension, a group of Fisher linear discriminant (FLD) classifiers needs to be assembled together, which causes the greatly enhancement of the computational cost. This is because Gabor feature is an overcomplete representation with a high redundant ratio. In addition, Gabor convolution brings a considerable time cost.

Accordingly, in this paper, the recently proposed shiftable complex directional filter bank (CDFB) transform [82,83] is investigated to capture the energy shiftable multiresolution and multidirectional information of the palmprint image. "Shiftable", namely translation invariance, was firstly defined by Simoncelli [84]. According to [84], the shiftability constraint is equivalent to 


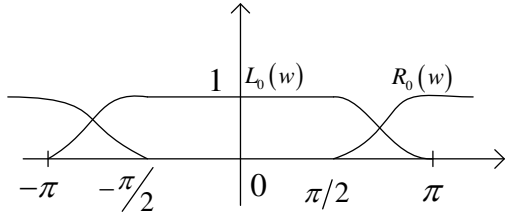

(a)

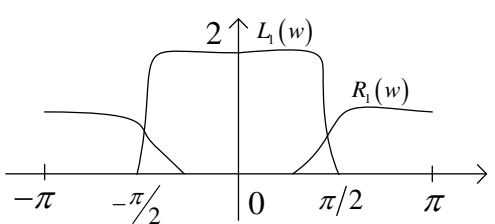

(b)

Fig. 4.3: Slices of the 2-D frequency responses of (a) $R_{0}(z)$ and $L_{0}(z)$, and (b) $R_{1}(z)$, and $L_{1}(z)$.

the constraint that the power of the transform coefficients in the subband is preserved when the input signal is shifted in position. The acceptable shiftable property of the CDFB decomposition has been demonstrated in detail in Ref. [82]. Compared with Gabor filtering, the CDFB transform maintains a much lower redundant ratio and computational complexity. The derived CDFB amplitude coefficients of complex-value subbands and the coarse subbands are further filtered independently by the uniform LBP operator which results in the corresponding LBP mapping matrixes [69]. To capture the spatial information of palmprint, each LBP mapping matrix needs to be divided into many subblocks which are then represented as LBP histogram independently. By concatenating all the histogram sequences, we get a shiftable and gray scale invariant local feature space. Finally, a Fisher Linear Discriminant (FLD) classifier [35] is learned in the statistical local feature space for palmprint recognition. Considering other multiscale and multidirectional transforms have been reported, including Gabor filter bank, dual-tree complex wavelet transform (DTCWT) [85], discrete Contourlet transform [35] and Nonsubsampled Contourlet transform (NSCT) [86], some comparative experimental studies are performed by fusing them with LBP, as can be seen in the experimental section.

The remainder of this paper is structured as follows. We present the proposed shift and gray scale invariant features in Sec. 4.2.3, containing three subsections. Sections 4.2.3.1 and 4.2.3.2 introduce the complex directional filter Bank (CDFB) and the uniform local binary pattern respectively. The proposed invariant feature extraction method is presented in Sec. 4.2.3.3. We investigate the palmprint identification performance with our proposed method in Sec. 4.2.4, together with other contrast experiments given as well. Section 4.2.5 concludes the paper and educes the future work. 


\subsubsection{Feature extraction using complex directional wavelet and}

\section{local binary pattern}

Generally, the palmprint image variations like non-uniform gray scale and minor translation are impossible to completely avoided, which is resulted from the illumination perturbation of capture condition and the nonideal preprocessing procedures. How to extract the shift and gray scale invariant features is a vital step for palmprint recognition successfully. In this section, our proposed approach extracting the shift and gray scale invariant local features is described, which combines the procedures of the shiftable complex directional filter bank (CDFB) transform [82,83] and the local binary pattern (LBP) [69] coding. Based on the proposed local features, FLD analysis classifier [35] is carried out for palmprint recognition, which is a supervised subspace learning technique. FLD searches for the projection axes on which the data points of different classes are far from each other while requiring data points of the same class to be close to each other. The FLD transformation matrix is trained in the PCA subspace, where the dimension that FLD kept is $C-1$ (Given $C$ is the total number of class). The Nearest-Neighbor based on Euclidean distance is employed for classification.

\subsubsection{The complex directional filter bank (CDFB) transform}

The (energy) shiftable complex directional filter bank (CDFB) is a recently proposed multiresolution and multidirectional decomposition method, which is carried out by a pyramidal dual-tree directional filter bank (PDTDFB) $[82,83]$. The structure of CDFB is illustrated in Fig. 4.2. As can be seen, the block $P$ is the so called PDTDFB, which consists of a Laplacian pyramid and a pair of directional filter banks (DFBs), designated as primal and dual DFBs. The Laplacian pyramid provides multiresolution image decomposition, where the signal is divided into two pairs: the coarse approximation (Point $L$ in Fig. 4.2) and the high frequency component (Point $H$ in Fig. 4.2). This high frequency component is then decomposed by a dual-tree of DFBs to produce the real and imaginary parts of the $2 n$ complex directional subbands. The directional filters of the dual DFB are Hilbert transforms of those of the primal DFB. The block $P$ is repeated at the low frequency output (Point $L$ ). At the front end, an undecimated two-channel FB $\left(L_{0}(z)\right.$ and $\left.R_{0}(z)\right)$ is used to separate the high frequency components near $( \pm \pi, \cdot)$ and $(\cdot, \pm \pi)$, which can potentially 

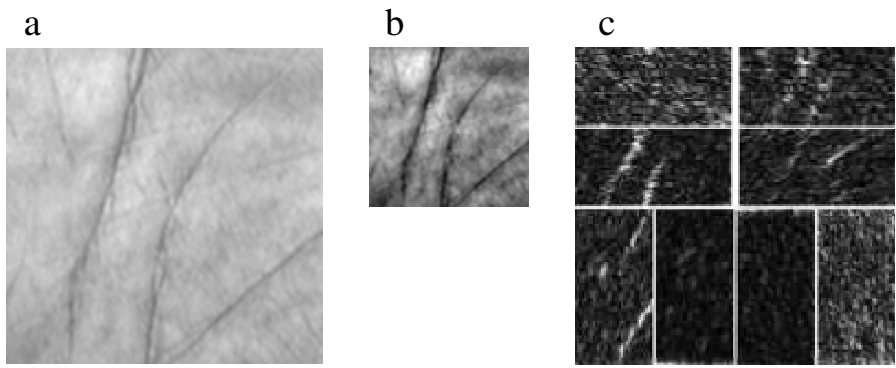

Fig. 4.4: An example of the CDFB decomposition with one level and eight directional bands: (a) a $128 \times 128$ palmprint image, (b) the coarse subband, and (c) the amplitude subbands of complex-valued coefficients.

cause aliasing in the dual-tree. The slices of 2-D frequency responses of $L_{0}(z)$ and $R_{0}(z)$ are shown in Fig. 4.3(a). To make the block $P$ satisfy the PR and non-aliasing conditions, the frequency response of $L_{1}(z)$ is designed within $[-\pi / 2, \pi / 2]^{2}$ (see Fig. 4.3(b)). The non-aliasing property achieved by the dualtree DFB leads to the energy shift-invariance or "shiftability" of the CDFB transform.

The image decomposition offered by the PDTDFB possesses some desirable properties. It is shiftable which makes the presentation stable with respect to signal translation, unlike some other multidirectional transforms including Contourlet transform [85] and Gabor filters. While compared with the shiftable image decompositions like dual-tree complex wavelet transform (DTCWT) [86] and the Nonsubsampled Contourlet transform (NSCT) [87], PDTDFB has a very low redundant ratio. In addition, PDTDFB provides arbitrarily high directional resolution without increasing the redundant ratio of the representation. Figure 4.4 shows an example of the CDFB decomposition with one level and eight directional bands for a palmprint image from the HongKong PolyU palmprint database [6].

\subsubsection{Local binary pattern (LBP)}

Local binary pattern (LBP) as a successful texture descriptor has been widely used for image representation in pattern recognition field. The original LBP operator $\left(L B P_{(8,1)}\right)[69]$ labels the pixels of an image by thresholding the $3 \times 3$ neighborhood of each pixel $f_{p}(p=0,1, \ldots, 7)$ with the center value $f_{c}$ and 

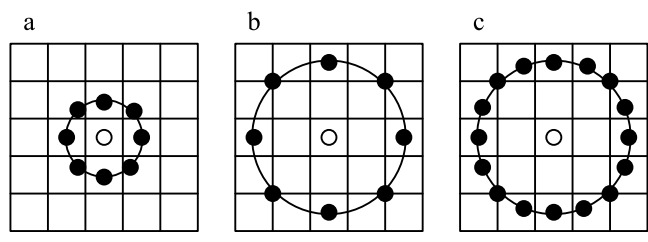

Fig. 4.5: Three examples of the extended LBP. (a) $(P=8, R=1)$, (b) $(P=8, R=2)$, and (c) $(P=16, R=2)$.

considering the result as a binary number:

$$
S\left(f_{p}-f_{c}\right)=\left\{\begin{array}{ll}
1, & f_{p} \geq f_{c} \\
0, & f_{p}<f_{c}
\end{array} .\right.
$$

Then, by assigning a binomial factor $2^{p}$ for each $S\left(f_{p}-f_{c}\right)$, the LBP pattern at the pixel is achieved as:

$$
\operatorname{LBP}_{(8,1)}=\sum_{p=0}^{7} S\left(f_{p}-f_{c}\right) 2^{p}
$$

which produces $256\left(2^{8}\right)$ different output values, corresponding to 256 different binary patterns (or called LBP codes). To deal with different scales of micro-texture, the original $L B P_{(8,1)}$ has been extended to $L B P_{(P, R)}$, where the notation $(P, R)$ denotes a neighborhood of $P$ equally spaced sampling points on a circle of radius of $R$ that forms a circularly symmetric neighbor set. See Fig. 4.5 for examples of the extended LBP operators. The $L B P_{(P, R)}$ operator produces $2^{P}$ different output values corresponding to the $2^{P}$ different binary patterns formed by the $P$ pixels in the neighbor set. It has been observed that certain LBP codes are fundamental, providing the vast majority of all patterns present in the observed textures. It is possible to use only a subset of the $2^{P}$ LBP codes to describe the texture of images. Accordingly, the uniform patterns are defined, denoted by $L B P_{(P, R)}^{u 2}$, which contain at most two bitwise transitions from 0 to 1 or vice versa when the binary string is considered circular.

After labeling an image with the LBP operator, a histogram of the labeled image can be achieved, which contains information about the distribution of the local micro-patterns, such as edges, spots and flat areas (as shown 


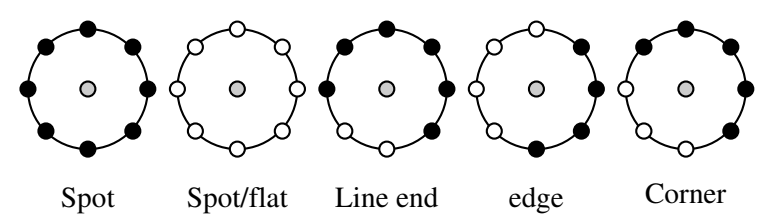

Fig. 4.6: Examples of texture primitives which can be detected by LBP (white circles represent ones and black circles zeros).

in Fig. 4.6), over the whole image, so the LBP histogram can be used to statistically describe image characteristics. When uniform patterns are used in the computation of the LBP histogram, each uniform pattern has a separate bin and all nonuniform patterns are assigned to a single bin. Accordingly, the histogram bin numbers are greatly reduced. For example, the number of bins for $L B P_{(8, R)}$ is 256 but 59 for $L B P_{(8, R)}^{u 2}, L B P_{(16, R)}$ is 65,536 but 243 for $L B P_{(16, R)}^{u 2}$.

Since just the signs of the differences $\left[S\left(f_{p}-f_{c}\right)\right]$ are considered, which are not affected by changes in mean luminance, the gray scale invariant feature is achieved after using the LBP coding.

\subsubsection{Shift and gray scale invariant feature extraction}

Inspired by the desirable properties of the shiftable complex directional filter bank (CDFB) transform and the uniform local binary pattern (LBP) coding, we extract the shift and gray scale invariant local features from palmprint image by processing them successively. However, the variations of parameter settings, including the scale resolution and directional resolution of CDFB transform, and the values of $P$ and $R$ for $L B P_{(P, R)}^{u 2}$, affect the palmprint performance to a great extent.

In this work, the scale resolutions ranging from 1 to 3 and directional resolutions at 4,8 , and 16 are considered respectively. 59-bin $L B P_{(8, R)}^{u 2}$ is selected to label the subband coefficients of CDFB transform. The optimal $R$, scale and directional resolution are experientially determined in Sec. 4.2.4.2.

To capture the spatial structure information, it is necessary to divide all the CDFB transform subbands into many subblocks, from which the $L B P_{(8, R)}^{u 2}$ histograms are extracted independently. In this paper, the partition is pyramidaland-square based, with the minimal subblock size of $16 \times 16$. For example, assuming the palmprint size of $128 \times 128$ is decomposed by CDFB transform 

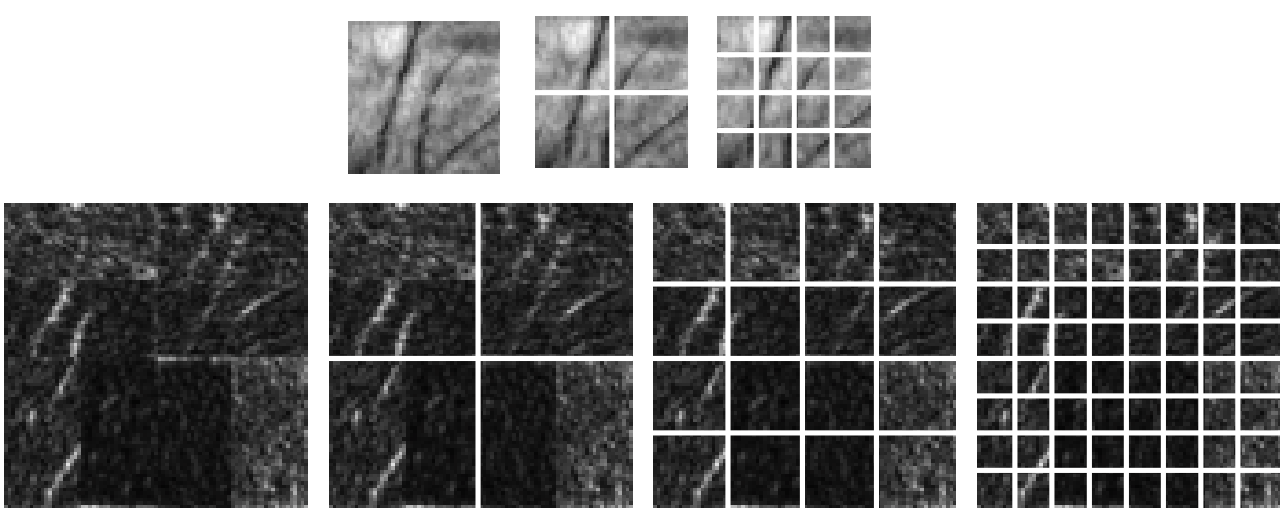

Fig. 4.7: The partition scheme for the coarse subband and eight high-frequency subbands, achieving 106 subblocks.

at one-scale resolution and eight directions, we will get one coarse subband of $64 \times 64$, and eight complex-valued subbands size of $32 \times 64$. By splicing together the magnitude coefficients of the eight subbands, we will get a square matrix size of $128 \times 128$. For the coarse subband and the resulting high-frequency magnitude matrices, quadtree-based partitions are processed independently. Finally, 106 subblocks are obtained, as shown in Fig. 4.7.

The histograms extracted from all the resulting subblocks are further concatenated into an enhanced sequence. For example, the achieved 106 subblocks will provide a spatial enhanced histogram of $6254(106 \times 59)$ bins, which builds a global description of the palmprint on both pixel level and regional level. These features form our proposed shift and gray scale invariant representation.

\subsubsection{Experimental results}

\subsubsection{Palmprint data}

All the experiments are conducted on the HongKong PolyU Palmprint Database [6], which has been the de facto standard database for evaluating the palmprint recognition technologies. It contains 7752 grayscale images in BMP image format corresponding to 386 different palms captured by CCD camera. The resolution of all the original palmprint images is $384 \times 284$ pixels at $75 \mathrm{dpi}$. They were captured on two separate occasions with different lighting conditions, at an interval of around two months. On each occasion, the subject 

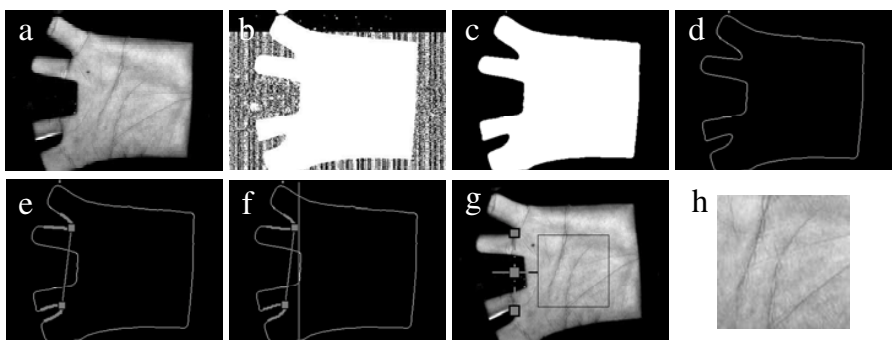

Fig. 4.8: Main steps of preprocess: (a) original image, (b) Gaussian lowpass filtering, (c) binarization and median filtering, (d) boundary tracing by Sobel operator, (e) locating reference point, (f) rotation angle computing, (g) image rotating by the computed angle, and (h) cropping ROI size of $128 \times 128$.
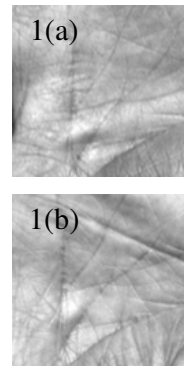
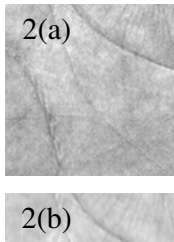
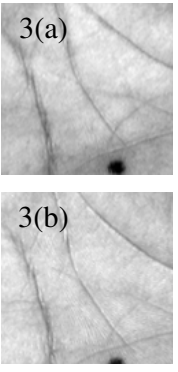
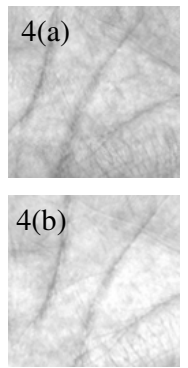
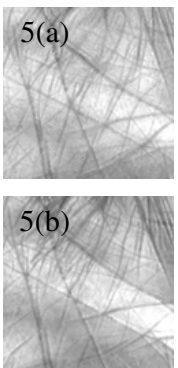

Fig. 4.9: Five typical palmprint images from different palms in the HongKong PolyU Palmprint Database. (a) and (b) denote the images which are captured from one same palm in the first and second sessions respectively.

was asked to provide about ten images, each of the left palm and the right palm. By performing the reprocessing steps illustrated in Fig. 4.8, palmprint is orientated and the region of interest (ROI) size of $128 \times 128$ is cropped. The corresponding cropped images of five typical palms in this database are shown in Fig. 4.9, in which (a) and (b) denote the samples captured from the same palm at the first and second sessions respectively.

All the experiments of our work were conducted on a personal computer with an Intel Pentium(R) Dual-Core Processor (E5200@2.50GHz) and 2GB RAM configured with Microsoft Windows XP and Matlab 7.5.

\subsubsection{Parameters selection and performance test}

Section 4.2.3 has given the architecture of the proposed method. The image needs to be firstly decomposed by the shiftable CDFB transform. However, the 
Table 4.1: Comparisons of the average identification accuracy (\%) with different parameters. For the CDFB transform, the scale level ranges from 1 to 3 , and the directional resolution is set to 4,8 and 16 respectively. For $L B P_{(P, R)}^{u 2}$ operator, $(P, R)=(8,1)$ and $(P, R)=(8,2)$ are both evaluated.

\begin{tabular}{|cl|ccc|}
\hline \multirow{2}{*}{ Directional resolution } & \multicolumn{3}{|c|}{ Scale resolution } \\
\cline { 3 - 5 } & & 1 & 2 & 3 \\
\hline \hline 4 & & & & \\
& $(P, R)=(8,1)$ & 97.25 & 82.27 & 67.93 \\
& $(P, R)=(8,2)$ & 99.19 & 93.45 & 82.87 \\
8 & & & & \\
& $(P, R)=(8,1)$ & 96.48 & 72.93 & 54.13 \\
& $(P, R)=(8,2)$ & 99.34 & 94.22 & 83.32 \\
16 & & & & \\
& $(P, R)=(8,1)$ & 94.72 & 58.24 & 32.86 \\
& $(P, R)=(8,2)$ & 99.23 & 88.69 & 64.27 \\
\hline
\end{tabular}

scale and directional resolution of CDFB transform are both adjustable. To determine the optimal settings of them for palmprint recognition, the identification accuracy, with the scale resolutions ranging from 1 to 3 and directional resolutions at 4, 8, and 16, are investigated respectively. About the 59-bin $L B P_{(8, R)}^{u 2}, R$ (set to 1 or 2 ) is selected to investigate for the best recognition performance.

For the experiments, all the palmprint samples are from the first session. Three images of each class are randomly selected to construct the training set and the last samples (in total 2731 images) form the testing set. Our system works in the identification mode, the decision is "whose palmprint data is this?" The performance is measured by the accurate identification rate. To evaluate the generalization performance, ten-run identification tests are executed. We report the average identification results, which are tabulated in Table 4.1. As can be seen, the recognition accuracy reduces sequentially as the scale level increase from 1 to 3 , and $R=2$ outperforms $R=1$ evidently. The best recognition performance $(99.34 \%)$ is achieved with the $L B P_{(8,2)}^{u 2}$ operators, and the one-scale decomposition of CDFB at eight-directional resolution.

\subsubsection{Effect of CDFB transform}

To verify the efficiency of CDFB in our proposed feature extraction framework, some other multi-scale and multi-directional transforms, including Gabor filter banks, dual-tree complex wavelet transform (DTCWT) [86], discrete Con- 
tourlet transform [85] and Nonsubsampled Contourlet transform (NSCT) [87], are carried out respectively before the LBP labeling.

The Gabor based recognition performance is generally dependent on the Gabor filter parameters. In this paper, the circular 2-D Gabor filter is used according to Ref. [12], which can be formulated as following:

$$
\begin{aligned}
G_{m, n}(x, y)= & \frac{1}{2 \pi \sigma^{2}} \exp \left\{\frac{-\left(x^{2}+y^{2}\right)}{2 \sigma^{2}}\right\} \\
& \times \exp \left\{2 \pi i\left(u_{m} x \cos \theta_{n}+u_{m} y \sin \theta_{n}\right)\right\},
\end{aligned}
$$

where $i=\sqrt{-1}, \sigma$ determines the bandwidth of the Gabor filter, which is commonly set to 5.6179 for palmprint feature extraction on the basis of Kong's experimental results [12]. $u_{m}$ is the frequency of the sinusoidal wave along the direction $\theta_{n}$ from the x-axis. In general, the Gabor based recognition performance is also involved with the number of used Gabor filters. In this paper, five scales are fixed as those used in Ref. [67], $u_{m}=0.2592 / \sqrt{2^{m}}$, $m=0,1, \ldots, 4$. The Gabor orientation is formulated as $\theta_{n}=\pi n / N, n=$ $0,1, \ldots,(N-1)$. And the considered orientation number $N$ is set to 4,6 and 8 respectively. Accordingly, three groups of Gabor filter bank are investigated, which include 20, 30 and 40 filters respectively.

In this group of experiments, discrete Contourlet transform and NSCT are both set to one-resolution and eight-directional decomposition. DTCWT, which has only six directional subbands, is set to one-resolution decomposition. Figure 4.10 shows an example of one palmprint decomposition by using these methods respectively. Here it should be noticed that only the magnitude parts of each transform are showed. The resulting subband coefficients are all further operated by the same partition scheme demonstrated in Sec. 4.2.3.3 and the same $L B P_{(8,2)}^{u 2}$ labeling method. The histograms are finally processed using the FLD technology like that for the proposed method.

For evaluating the performances of the considered multiscale and multidirectional transforms, five images of each palm collected in the first session are randomly chosen to construct the training set, and all the samples in the second session (in total 3863 samples) are used for testing. Table 4.2 shows the overall performances over ten run executions. As can be seen, the average recognition accuracies of the original intensity based and the Contourlet based methods are both lower ( $88.26 \%$ and $88.93 \%$ ), because their extracted features are not shift invariant. The identification accuracy of the NSCT based method 
(1)

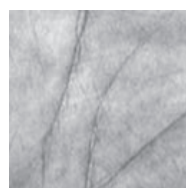

(2)

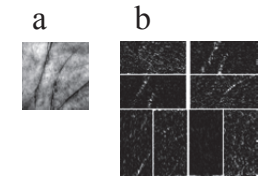

(3) a

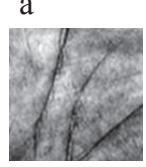

b

(4)
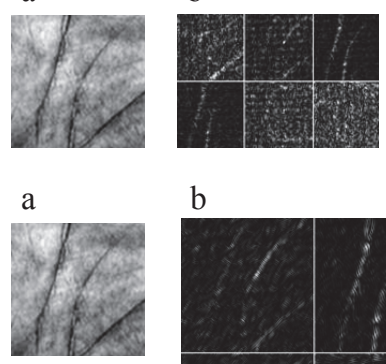

b

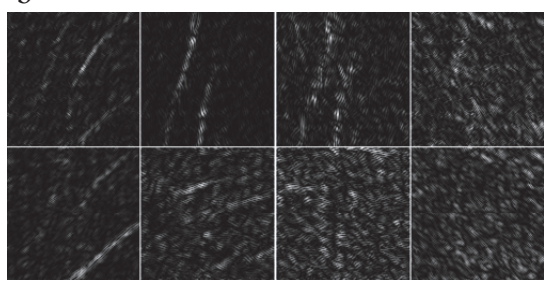

(5)
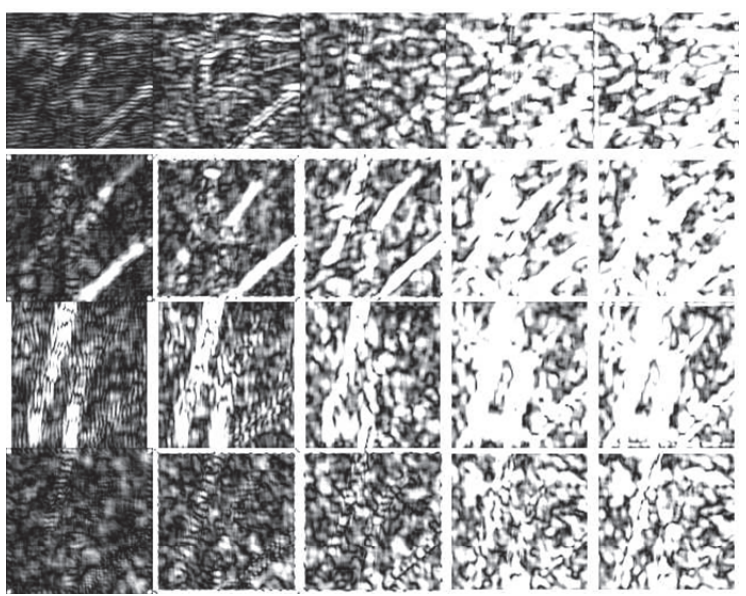

Fig. 4.10: Visualization of different multiscale and multidirectional decompositions. (1) An example of original palmprint sample; (2) Contourlet decomposition at one scale and eight directions: (a) Coarse subband and (b) high-frequency amplitude subbands; (3) Dualtree complex wavelet transform (DTCWT) at one scale: (a) Coarse subband and (b) highfrequency amplitude subbands; (4) Nonsubsampled Contourlet transform (NSCT) at one scale and eight directions: (a) Coarse subband and (b) high-frequency amplitude subbands; (5) Gabor magnitude coefficients at five scales and four orientations. 
Table 4.2: Comparisons of the identification performance when using different multiscale and multidirectional transforms for the proposed palmprint identification framework.

\begin{tabular}{|l|ccc|c|c|c|c|c|}
\hline & \multicolumn{3}{|c|}{ Gabor } & & & & & \\
\cline { 2 - 9 } & 20 & 30 & 40 & CDFB & Intensity & DTCWT & Contourlet & NSCT \\
\hline \hline Recognition accuracy (\%) & 91.05 & 93.90 & 94.72 & 93.31 & 88.26 & 92.35 & 88.93 & - \\
Redundancy ratio & 20 & 30 & 40 & 1.25 & 1 & 2.5 & 1.25 & 9 \\
Histogram bin length & 5900 & 8850 & 11,800 & 6254 & 5015 & 12,449 & 6254 & 45,135 \\
Histogram extraction time (s) & 0.211 & 0.270 & 0.367 & 0.087 & 0.047 & 0.120 & 0.073 & 5.052 \\
\hline
\end{tabular}


is not given here, because the process of histogram extraction from each image costs too much time $(5.052 \mathrm{~s})$, which is not desirable for on-line identification system. Compared with DTCWT, also energy shiftable, CDFB provides less feature redundancy ratio (1.25), less histogram bins (6254), and takes too less time to compute $(0.087 \mathrm{~s})$. Further, the accuracy of CDFB is $0.95 \%$ higher than that of DTCWT, since DTCWT provides only six directional decompositions while CDFB provides eight. The performances of Gabor filter bank based approach are involved with the number of used filters. As the filter number increases, the identification accuracy raises. When 20 Gabor filters are used, the identification accuracy is $2.26 \%$ lower than that of the proposed CDFB based method. When the number of Gabor filters is set to 30 and 40, the accuracy of CDFB is $0.59 \%$ and $1.41 \%$ lower respectively, but still comparable. Furthermore, Gabor transform leads to large information redundancy. The redundancy ratio is $S \times N$, given $S$ is the scale resolution and $N$ the orientational resolution. These over-complete data will greatly increase the storage burden of the system. In this paper, we have down-sampled each Gabor coefficient matrix by the ratio of 4:1. Otherwise, their resulting histogram bins will be too many to directly execute the FLD classification (out of memory) on our used computer system. Moreover, Gabor transforms cost much more time. By considering the key factors of an identification system synthetically including the accuracy, speed, and storage requirement, we can conclude that the proposed method based on CDFB performs best.

\subsubsection{LBP versus CLBP for the proposed method}

In this section, we conduct a series of experiments to evaluate the identification performance when using Completed LBP (CLBP) [78] instead of LBP to encode the CDFB transform aptitudes for the proposed feature extraction framework, since several variants of LBP have been reported efficient for texture classification.

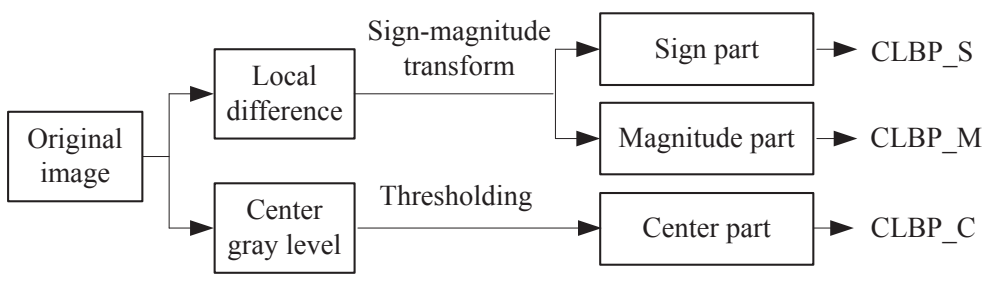

Fig. 4.11: Diagram of Completed LBP (CLBP). 
In Ref. [78], Guo et al. develop the CLBP scheme, whose framework is illustrated in Fig. 4.11. Three operators, namely CLBP_C, CLBP_S and CLBP_M, are proposed to encode local center gray level, local sign difference and local magnitude difference features, respectively, which could be further combined jointly or hybridly to generate different completed LBP operators. For example, by calculating a joint 2-D histogram of one another, CLBP_S/M, CLBP_M/C and CLBP_S/C could be formed respectively. In another way, two histograms of CLBP_S and CLBP_M could be concatenated directly, denoted by CLBP_S_M. Further, a 2-D joint histogram, resulted from CLBP_M/C, can be converted into one 1-D histogram and then concatenated with the histogram of CLBP_S to generate a new joint histogram, denoted by CLBP_S_M/C.

The experimental setups are as follows:

(1): Parameters: There are several adjustable parameters for CLBP, which are the same as LBP method. Motivated by the experimental results illustrated in Sec. 4.2.4.2, we use $C L B P_{-} S_{(8,2)}^{u 2}$ and $C L B P \_M_{(8,2)}^{u 2}$ to encode the CDFB magnitude responses in this group of experiments. The image is partitioned in the same way as described in Fig. 4.7.

(2): Palmprint data: To further evaluate the performances of different CLBP operator encoding approaches, the experiments are executed in two different palmprint data sets, denoted as Set I and Set II respectively. For Set I, all samples are collected in session one. Three samples are randomly chosen from each class to construct training data set and all the remaining images (in total 2731) are used as testing set. For Set II, Five samples from each class are randomly selected in session one to form the training data set, and all the samples in session two are for test. Accordingly, compared with Set I, the training samples and the test ones in Set II differ significantly in illumination.

(3): Classifier: In Ref. [78], the nearest neighborhood (NN) classifier with the Chi-square distance is used to measure the dissimilarity between two histograms. While in our proposed feature extraction framework, the histogram features need to be processed firstly by FLD and then the NN classifier is used. Accordingly, we conduct the experiments in both of two ways.

Table 4.3 lists the experimental results. The CLBP_S operator is the same as the original LBP operator used in our proposed method. The identification accuracies of CLBP_S/M are not shown in Table 4.3 because the dimension of its resulting histogram vector is too high to execute FLD computation with our used computer system. From Table 4.3, we can conclude that CLBP_S 
Table 4.3: Comparisons of the identification performance among different CLBP operators when used for the proposed palmprint identification framework.

\begin{tabular}{|l|c|cc|cc|}
\hline \multirow{2}{*}{ Method } & Histogram & \multicolumn{2}{|c|}{ Set I: Identification } & accuracy (\%) & \multicolumn{2}{|c|}{ Set II: Identification accuracy (\%) } \\
\cline { 3 - 6 } & bin length & Chi-square & FLD & Chi-square & FLD \\
\hline \hline CLBP_S (Ours) & 6,254 & 99.52 & 99.32 & 89.92 & 93.31 \\
CLBP_M & 6,254 & 98.13 & 97.03 & 76.98 & 86.10 \\
CLBP_S_M & 12,508 & 98.94 & 99.34 & 75.04 & 93.28 \\
CLBP_S/M & 368,986 & - & - & - & - \\
CLBP_S/C & 12,508 & 99.19 & 99.58 & 77.65 & 93.61 \\
CLBP_M/C & 12,508 & 98.21 & 98.46 & 65.10 & 89.47 \\
CLBP_S_M/C & 18,762 & 99.18 & 99.74 & 75.89 & 94.26 \\
\hline
\end{tabular}


features are more effective than CLBP_M, i. e. the sign components of the local difference are more informative than the magnitude components, which has been intensively illustrated in Ref. [78]. Besides, the results tell us that for the proposed feature extraction framework, encoding CDFB transform magnitudes using CLBP instead of LBP does not make the identification performance improved noticeably while it does generate more histogram bins for feature representation. Meanwhile, learning based classifier can achieve much higher identification accuracy than Chi-square based template matching classifier, especially when the samples are under different illumination conditions.

\subsubsection{Comparisons with local Gabor pattern operators}

Recently, many methods based on fusing Gabor images and LBP have been proposed for face recognition and other object representation. Generally, Gabor magnitude or phase responses are encoded by designing different local operators to form variant local Gabor patterns. For example, by encoding Gabor magnitude and phase coefficients via LBP operator, LGBP_Mag [70] and LGBP_Pha [79] have been proposed respectively. By using the local XOR pattern (LXP) operator to encode the real and imaginary parts of Gabor complex response, local Gabor phase pattern (LGPP) has been proposed [80], denoted by Re_LGPP and Im_LGPP respectively. Different from LGPP, GGPP has been proposed to represent orientation patterns, which forms one 8-bit binary string to represent each pixel by concatenating the real or imaginary quadrant-bit codes of different orientations for a given frequency (denoted by Re_GGPP and Im_GGPP). In addition, Xie et al. [81] proposed local Gabor XOR pattern (LGXP) by quantizing the Gabor phase in each local region firstly, and then encoding the quantized phases by LXP operator. Figure 4.12 gives an illustration of different local Gabor pattern operators.

For the proposed method in the paper, we actually encode the CDFB transform coefficients, instead of Gabor responses, using LBP operator to achieve a new local pattern to represent palmprint image. To validate the effectiveness of this scheme, we compare its performance with several local Gabor pattern based methods, in terms of identification accuracy, resulting histogram bin length, and histogram extraction time cost. The experimental results are listed in Table 4.4. Here, the histogram of Gabor phase pattern (HGPP) is formulated as HGPP $=($ Re_GGPP, Im_GGPP, Re_LGPP, Im_LGPP). The featurelevel fusion of LGBP_Mag and LGXP is denoted as F[LGBP_Mag+LGXP], and the score-level fusion denoted as S[LGBP_Mag+LGXP]. For more detailed 


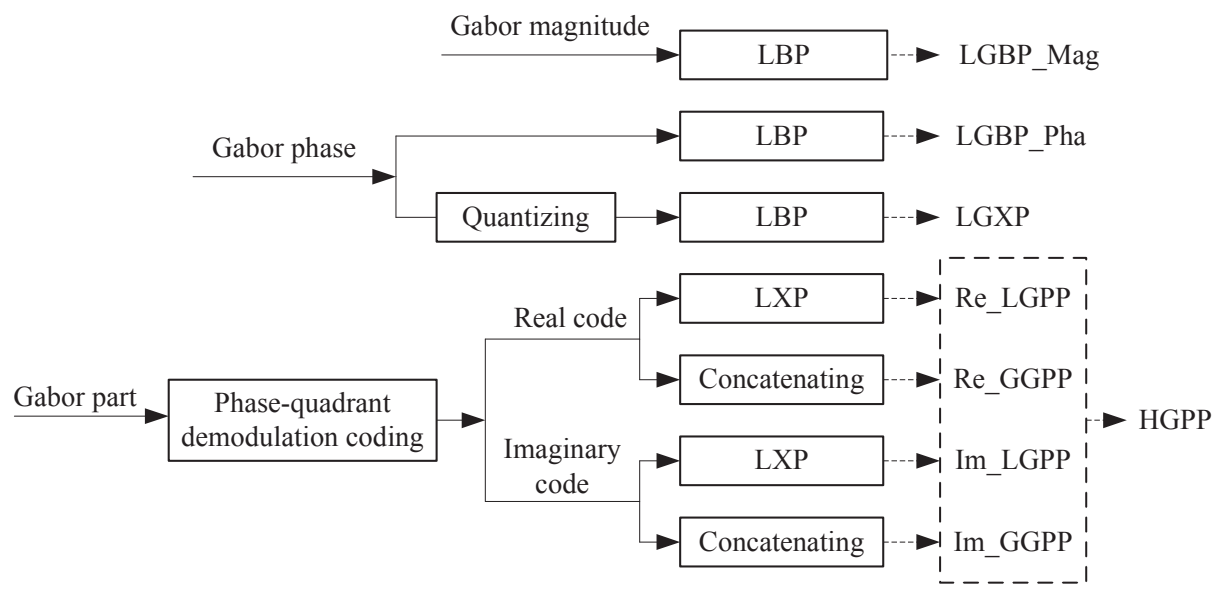

Fig. 4.12: Illustration of different local Gabor pattern operators.

information, please refer to Refs. [80] and [81].

In this group of experiments, the palmprint data and the corresponding parameters for each method are set as following:

(1): Parameters: For each local Gabor pattern based method listed in Table 4.4, five-scale and eight-orientation Gabor filter bank, whose parameters are set the same as illustrated in Sec. 4.2.4.3, is used for feature extraction, and every Gabor coefficient matrix is not downsampled. Each local Gabor pattern matrix is partitioned into four $(2 \times 2)$ non-overlapping subblocks. For each subblock, histogram feature is computed and then FLD is executed to reduce the feature dimension. Finally, four parts of the resulting feature sequences are concatenated as the final feature template. The nearest neighbor (NN) classifier is used for palmprint identification.

(2): Palmprint data: Five samples from each class in session one are randomly chosen to construct the training set, and all the images collected in session two are used for testing. This process is executed ten runs, and then the average identification accuracy of each method is reported in Table 4.4.

From Table 4.4, we can see that the local Gabor patterns based methods can achieve higher identification accuracy than our proposed approach. This is because the high redundancy information of Gabor images contributes to the feature dissimilarity ability. But, the high Gabor redundancy generates more histograms, which leads to high-dimensionality problem for learning FLD matrices and makes the final feature size large. Meanwhile, the dimension size would exponentially grow if the subblocks are partitioned further. Besides, 
Table 4.4: Comparisons of the identification performance between different local Gabor pattern operators and the proposed method.

\begin{tabular}{|l|c|c|c|c|}
\hline Method & Identification accuracy (\%) & $\begin{array}{c}\text { Final } \\
\text { feature length }\end{array}$ & $\begin{array}{c}\text { Histogram } \\
\text { bin length }\end{array}$ & $\begin{array}{c}\text { Histogram extraction } \\
\text { time (s) }\end{array}$ \\
\hline \hline Ours & 93.31 & 385 & 6254 & 0.08 \\
Re_LGPP & 97.10 & 1540 & 40,960 & 0.28 \\
Im_LGPP & 97.22 & 1540 & 40,960 & 0.27 \\
Re_GGPP & 85.97 & 1540 & 5120 & 0.13 \\
Im_GGPP & 91.37 & 1540 & 5120 & 0.13 \\
HGPP & 98.40 & 1540 & 92,160 & 0.62 \\
LGBP_Mag & 95.86 & 1540 & 40,960 & 0.48 \\
LGBP_Pha & 97.67 & 1540 & 40,960 & 0.44 \\
LGXP & 98.24 & 1540 & 40,960 & 0.51 \\
F[LGBP_Mag+LGXP] & 98.17 & 3080 & 81,920 & 0.73 \\
S[LGBP_Mag+LGXP] & 98.12 & 81,920 & 0.99 \\
\hline
\end{tabular}


Gabor convolution costs more time. To sum up, the proposed method outperforms local Gabor patterns based approaches in terms of high computational speed and less storage space requirement, which makes it possible to be used in an identification system with more registered users.

\subsubsection{Comparisons with other methods}

Except comparing our methods with other local Gabor pattern methods, as shown in Table 4.4, comparisons are also made with other state-of-the-art results reported on the palmprint data sets, including PalmCode [12], and $\mathrm{G}(2 \mathrm{D})^{2}$ PCA [67]. PalmCode is a typical coding based method, which encodes the Gabor phase information of the palmprint and represents the image with two bit-planes size of $128 \times 128$. The Hamming distance is used for the palmprint matching, which is not rotation invariant. For obtaining the final matching score between two samples, the bit-feature matrices are commonly translated horizontally and vertically and matched many times. The minimum matching score obtained from the translated matchings will be considered to be the final matching score. Obviously, the identification accuracy will be heavily dependent on the range of translation. In this paper, to evaluate the identification performance of PalmCode, the ranges of image translation are set to $0,[-1,1]$, and $[-2,2]$ respectively. The used Gabor filter parameters are set as following: $\theta=\pi / 4, u=0.0916, \sigma=0.5179 . \mathrm{G}(2 \mathrm{D})^{2} \mathrm{PCA}$ is based on a group of Gabor magnitude coefficients. A bank of multiresolution and multidirectional filters is used, which derives a Gabor feature space of high dimensionality. To reduce the dimension, two steps of Principal Component Analysis (PCA) projections are carried out in both row and column directions respectively. It should be noticed that for $\mathrm{G}(2 \mathrm{D})^{2} \mathrm{PCA}$ method, the Gabor coefficients are downsampled by the ratio of 4:1. Otherwise, the Gabor feature dimensionality will be too high to be processed further in our experimental system. There groups of Gabor filter bank including 20, 30, and 40 filters respectively are used, whose parameters are set the same as those described in Sec. 4.2.4.3.

Because the palmprint images from the first and second sessions have been captured in different illumination conditions. We conduct the experiments in two different data sets, Set I and Set II, which have be illustrated in Sec. 4.2.4.4.

The experimental results are listed in Table 4.5. We report the average identification accuracies of ten-run executions for all the considered methods. For 
Table 4.5: Identification performance comparisons with other methods.

\begin{tabular}{|c|c|c|c|c|c|c|c|c|}
\hline & \multicolumn{3}{|c|}{ PalmCode } & \multicolumn{3}{|c|}{$\mathrm{G}(2 \mathrm{D})^{2} \mathrm{PCA}$} & \multirow[t]{2}{*}{ Ours } \\
\hline & & 0 & {$[-1,1]$} & {$[-2,2]$} & 20 & 30 & 40 & \\
\hline \multirow[t]{3}{*}{ Set I } & & & & & & & & \\
\hline & Identification accuracy (\%) & 89.54 & 99.13 & 99.91 & 98.65 & 98.60 & 98.76 & 99.32 \\
\hline & Identification time (s) & 0.563 & 5.395 & 14.703 & 0.107 & 0.168 & 0.215 & 0.022 \\
\hline \multirow{3}{*}{ Set II } & & & & & & & & \\
\hline & Identification accuracy (\%) & 75.51 & 95.14 & 98.31 & 81.53 & 81.44 & 81.42 & 93.31 \\
\hline & Identification time (s) & 0.937 & 8.981 & 24.887 & 0.151 & 0.222 & 0.519 & 0.036 \\
\hline & Redundancy ratio & \multicolumn{3}{|c|}{-} & 20 & 30 & 40 & 1.25 \\
\hline & Feature size (Bytes) / length ${ }^{*}$ & \multicolumn{3}{|c|}{ 2,048 Bytes } & 6,840 & 10,260 & 13,680 & 385 \\
\hline & Feature extraction time (s) & \multicolumn{3}{|c|}{0.005} & 0.159 & 0.226 & 0.289 & 0.095 \\
\hline
\end{tabular}

${ }^{*}$ For the vector feature, storage requirement is commonly described by the feature length. But for the bit-plane feature, the storage requirement is generally described by the feature size in Bytes. 
each one sample, the feature extraction time cost and the identification time cost are both investigated. Besides, the final feature length and the redundancy ratio of transform feature are also listed for comparison. As can be seen, compared with $\mathrm{G}(2 \mathrm{D})^{2} \mathrm{PCA}$, the proposed method achieves higher identification accuracy with much less computational cost. When compared with PalmCode, the identification accuracy of the proposed method is distinctly higher only when PalmCode implements no translation for obtaining the matching score. Besides, PalmCode has advantages in less feature extraction time cost, smaller final feature size, and less feature redundancy ratio. However, PalmCode costs too much time to identify one sample. And the more registered classes and training samples per class, the more time Palmcode costs for identifying one test sample. There are both 386 palm classes, 1158 training samples in Set I and 1930 ones in Set II. For Set II, the identification time for one image is up to $5.395 \mathrm{~s}$ when the range of translation is $[-1,1]$. For achieving the performance of $98.31 \%$, the system will have to cost 24.887 s to determine one palmprint's identity. In contrast, the proposed method only costs $0.022 \mathrm{~s}$ and $0.036 \mathrm{~s}$ to determine one's identify. Accordingly, for a verification system, which executes one-to-one matching, Palmcode is suitable. But for an identification system with large registered users, one testing sample will have to match with all the training samples (one-to-many matching), Palmcode fails because it will cost too much time. So the proposed method is more promising to be used in the identification system due to its higher computational speed and less storage requirement.

\subsubsection{Conclusion and future work}

This paper presents a novel approach for extracting features for palmprint identification. By combining the shiftable complex directional filter bank transform (CDFB) and the local binary pattern (LBP) coding, we get the shiftable and gray scale invariant local features. Compared with the other multiscale and multidirectional transforms, CDFB outperforms in terms of higher identification accuracy, less storage requirement, and lower computational complexity. In addition, the lower redundancy information of CDFB transform generates less LBP histogram bins, which makes it cost less when carrying out the supervised learning process like FLD, to achieve higher identification accuracy.

Compared with the current state-of-the-art palmprint methods, the outstanding advantages of the proposed approach consist in its higher execution speed 
and lower storage requirement. However, the proposed method does not perform that well when the training and testing samples are collected under different illumination conditions. In future, we need to do more research on fusing CDFB transform (or other more efficient low-redundancy multiscale and multidirectional transforms) and local pattern operator features to extract more discriminative information for palmprint identification.

\subsection{Chapter conclusion}

In this chapter, a texture presentation for palmprint recognition is proposed, which is based on the catenation of the CDFB transform and the LBP operator. With regards to the research objective which we formulate in Section 1.3.3, this chapter aims to a real-valued feature representation for an unprotected recognition system. The proposed representation performs better than those local Gabor pattern based methods and some state-of-the-art palmprint recognition methods by higher identification accuracy, less storage requirement and lower computational complexity, due to its lower redundancy. However, the recognition accuracy decreases when the compared images differ in illumination conditions. 
${ }_{\text {Chapter }}$

\section{Fourier spectrum of PalmCode}

\subsection{Chapter introduction}

PURPOSE. Different from Chapters 2, 3 and 4, this chapter investigates the pixel-based transform features for palmprint representation, instead of regionbased statistical features. The classical palmprint phase code (PalmCode) is further studied, because of its high discriminative ability and robustness to illumination variation. PalmCode together with other advanced coding-based approaches are sensitive to image translation and rotation, because the code feature templates generated by these methods require pixel-to-pixel matching. To cope with this problem, the general strategy is shifting and rotating the templates and then matching multiple times for similarity score. Consequently, it results in poor matching speed. Thus, in this chapter we present a novel palmprint representation based on further processing PalmCode by the Fourier transform and the horizontal and vertical two-dimensional principle component analysis $\left((2 \mathrm{D})^{2} \mathrm{PCA}\right)$, due to their translation invariance property.

CONTENTS. In this chapter, a real-valued palmprint representation is proposed, which is denoted by Fourier spectrum of PalmCode. In Section 5.2.3, a brief review of PalmCode is given. PalmCode represents a palmprint image by two binary code matrices, which is sensitive to image translation. To cope with it, we transform the code matrices into their corresponding Fourier 


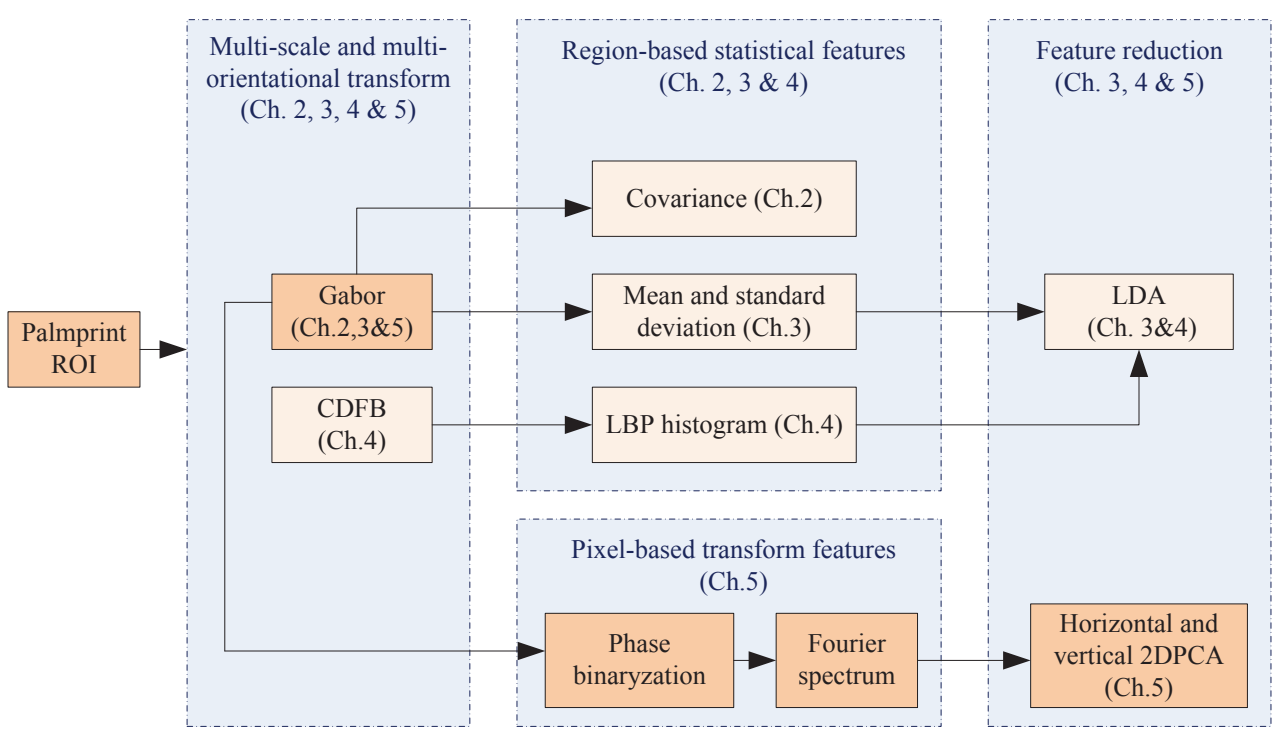

Fig. 5.1: Block diagram of our designed system, highlighting the context of Chapter 5 and its referred blocks.

magnitude spectrum, since a shift in the time domain causes no change in the Fourier magnitude spectrum. The detailed process is presented in Section 5.2.4. Subsequently, the resulting Fourier spectrum matrices are further processed by the two-dimensional principle component analysis horizontally and vertically, which we denote as $(2 \mathrm{D})^{2} \mathrm{PCA}$. The $(2 \mathrm{D})^{2} \mathrm{PCA}$ is applied not only for feature reduction, but also for alleviating the negative effect resulting from image translation. Section 5.2.5 presents the proposed algorithm for palmprint representation and recognition. Experiments are executed over the HongKong PolyU palmprint database. The system parameters are experimentally determined. Comparisons with the classical coding-based palmprint recognition methods and the other Gabor-based methods are illustrated. In the context of system diagram, the content of this chapter and its referred blocks are highlighted in Figure 5.1.

PUBLICATION(s). The content of Section 5.2 of this chapter has been published in [88]. 


\subsection{Fourier spectrum of PalmCode as representa-}

\section{tion}

\subsubsection{Abstract}

Study on automatic person recognition by palmprint is currently a hot topic. In this paper, we propose a novel palmprint recognition method by transforming the typical palmprint phase code feature into its Fourier frequency domain. The resulting real-valued Fourier spectral features are further processed by horizontal and vertical 2DPCA method, which turns out to be highly efficient in terms of computational complexity, storage requirement and recognition accuracy. This paper also gives a contrast study on palm code and competitive code under the proposed feature extraction framework. Besides, experimental results on the Hongkong PolyU Palmprint database demonstrate that the proposed method outperforms many currently reported local Gabor pattern approaches for palmprint recognition.

\subsubsection{Introduction}

Nowadays, recognition of individuals by means of biometric characteristics is becoming increasingly familiar and accepted. The selection of biometrics is commonly application-dependent [31,64]. Palmprint, extracted from person's hand, has been recognized as a means of measurement that can uniquely represent a person [89]. It has been a long history since the palmprints found on the crime scene were used for forensic investigation. Recently, palmprint is being investigated intensively for personal recognition in different real-time application system, such as access control, network security, and social security. Compared with the fingerprints, palmprints have more rich features which are less likely to be destroyed and forged. Besides, palmprints can be captured with a much lower resolution imaging sensor (less than $100 \mathrm{dpi}$ ), which leads to be more efficient [90]. The online palmprint capture devices are mainly based on CCD camera or digital scanner. More recently, the real time multispectral palmprint capture device has also been developed [91,92]. In addition to the efficient palmprint acquisition, robust palmprint representation is another key issue for the success of palmprint recognition application. 
The algorithms proposed for online palmprint recognition (including verification and identification), are generally divided into three main classes: subspace learning methods, texture energy feature extraction, and coding based methods. Among them, coding based methods are deemed to be the most promising due to their high recognition accuracy and small feature size, which typically involve steps of filter bank selection, coding scheme design and template matching approach. The popular filters include Gabor, Gaussian, and other self-designed ones. The phase, orientation, and magnitude information are generally regarded as encoded objects. The coding rules are usually flexible and simple, and the obtained code features should be robust and provide high discriminative ability. PalmCode [8] encodes the phase of Gabor filtered responses into binary features. FusionCode [93] used a fusion rule at feature layer to further improve PalmCode. DoG code method [42] first convolves the image using two-dimensional Gaussian filter and then encodes the zerocrossing information of horizontal and vertical gradient values, respectively. OrdinalCode [94] encodes the outputs of the elliptical Gaussian filters. The schemes encoding the orientation of palm lines are also popular due to its stability which includes competitive code (CompCode) [37], binary orientation cooccurrence vector (BOCV) code [41], palmprint orientation code (POC) [38], robust line orientation code (RLOC) [39], and so on [40,95]. However, coding based methods require the pixel-to-pixel paired matching between enrollment and query templates. In order to offset the inter-class translation variation, one template has to be shifted in some range and the matching score computation is proceeded multiple times to finally determine the identity of one query palmprint, which is time costly. Refer to Ref. [31], the matching speed of PalmCode is $1.7 \mathrm{~ms}$ when the system works under the verification mode and the algorithm is implemented in Visual $\mathrm{C}++6.0$ on a $\mathrm{PC}$ using Intel Pentium III processor (500MHZ). For identification, if the database contains 100 persons and three palmprint images are registered for each subject, the total identification time is about 1.1s. As can be seen, the matching speed of coding based methods is not desirable for the identification system with large registered database. This issue has also been addressed in some other reported literatures. In order to deal with it, Jia [96] applied Tensor LPP on their extracted directional representation. However, it can not provide translation invariant performance by reducing the dimension of integer codes directly. Yue [97] proposed an approach to speed up the coding based palmprint recognition method by building a template tree to perform fast nearest neighbor searching. For both of these methods, the final classification is still based on pixel-to-pixel code template matching by Hamming distance.

Accordingly, in this paper, we aim to further study the code features for 
palmprint representation. The following three issues are mainly considered:

Firstly, about the filter selection, Gabor, Gaussian, and the second derivative of Gaussian filter have been evaluated based on several coding based methods in Ref. [98] and their experimental results show that the Gabor filter is superior. Besides, it is well known that Gabor representation can provide optimal localization of image details in a joint spatial and frequency domain [62]. Concerning these conclusions, the Gabor filters are chosen for our proposed feature extraction method in this paper.

Secondly, about the encoded object, we employ the phase information. Gabor phase, as a discriminating information source, has been successfully used in pattern recognition field. Besides PalmCode and IrisCode, kinds of local Gabor phase pattern operators [79-81] are proposed as well based on the combination of the spatial histogram and the Gabor phase pattern encoding scheme. These methods involve a group of Gabor filters (in general 40 Gabor filters) to extract the multi-scale and multi-orientation information, which is an overcomplete representation with a high redundant ratio. The high dimensionality of the local Gabor phase pattern histograms leads to high computational cost and large storage requirement. To deal with this problem, this paper proposes a new method, which uses only two Gabor filters while provides higher recognition performance.

Thirdly, instead of representing palmprint image by the code features directly, we transform the code feature matrices into their Fourier frequency fields since a shift in the time domain causes no change in the Fourier magnitude spectrum. The resulting spectral features not only keep the property of powerful discriminative ability of PalmCode features, but also make it possible to extend the application field of coding based features. Because the dissimilarity measurement of the original coding based features is generally based on pixel-to-pixel matching by using Hamming or angular distances, which limits their application to unsupervised template matching mode, while the realvalued spectral features might be processed further by the currently reported supervised learning algorithms, which probably achieves higher recognition performance.

Taking all the above factors into consideration, a novel Fourier spectral representation of PalmCode (DFT_PalmCode) is proposed for palmprint recognition in this paper, whose flow chart is shown in Fig. 5.2. We firstly transform the typical palmprint phase code feature into its Fourier frequency domain by discrete Fourier transform (DFT). The resulting real-valued Fourier spectral features are then processed by the horizontal and vertical two-dimensional 
principal component analysis $\left((2 \mathrm{D})^{2} \mathrm{PCA}\right)$ method. The translation invariant property of Fourier transform and $(2 \mathrm{D})^{2} \mathrm{PCA}$ dimensionality reduction method help alleviating the within-class image translations to some extent. Compared with the original PalmCode method, the proposed feature extraction approach operates two pairs of Gabor filtering phase responses, which improves the recognition performance evidently. This paper also gives a contrast study on CompCode under the proposed feature extraction framework. For CompCode, palm line orientation information is encoded into bits. Furthermore, our experimental results demonstrate that the proposed method outperforms many currently reported local Gabor pattern operators for palmprint recognition by higher accuracy, lower computational cost and less storage requirement.

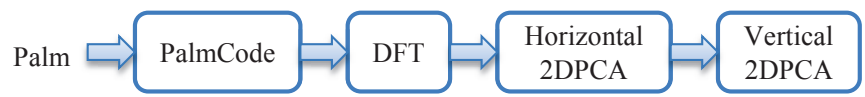

Fig. 5.2: Flow chart of the proposed method. (DFT - discrete Fourier transform; 2DPCA two-dimensional principle components analysis)

This paper is organized as follows. In Sec. 5.2.3, we give a short review of PalmCode. Section 5.2.4 illustrates the proposed Fourier spectral representation in details. In Sec. 5.2.5, the $(2 \mathrm{D})^{2} \mathrm{PCA}$ method is described for the dimensional reduction of the proposed spectral features, which helps alleviating the negative effects due to image translation. The experimental results will be demonstrated in Sec. 5.2.6. The final part is about the conclusions.

\subsubsection{Review of PalmCode}

The Gabor phase quadrant demodulation coding method is proposed by Daugman for iris recognition in 1993 [99], which is then reported successful for palmprint representation in Ref. [12]. For ease of presentation, this feature extraction method is commonly named by IrisCode, PalmCode or Daugman's method.

For PalmCode, a circular 2-D Gabor filter is used to firstly convolve the original 
gray palmprint image, which has the following general form:

$$
\begin{aligned}
g(x, y, u, \theta, \sigma) & =\frac{1}{2 \pi \sigma^{2}} \exp \left\{\frac{-\left(x^{2}+y^{2}\right)}{2 \sigma^{2}}\right\} \\
& \times \exp \{2 \pi i(u x \cos \theta+u y \sin \theta)\} .
\end{aligned}
$$

Where $i=\sqrt{-1}, u$ is the frequency of the sinusoidal wave along the direction $\theta$ from the x-axis, and $\sigma$ specifies the Gaussian envelope along $x$ and $y$ axes, which determines the bandwidth of the Gabor filter. In practice, a Gabor function with a special set of parameters $\{u, \theta, \sigma\}$, is transformed into a discrete Gabor filter. In order to provide more robustness to brightness, the Gabor filter is turned to zero direct current (DC) denoted by $\tilde{g}(x, y)$. Following the experimental setting in Ref. [12], $\sigma$ is set to 5.6179 .

Given an image $f(x, y)$ of size $M \times N$, its Gabor filtered images are defined as:

$$
G(x, y)=\sum_{x_{1}} \sum_{y_{1}} f\left(x_{1}, y_{1}\right) \cdot \tilde{g}\left(x-x_{1}, y-y_{1}\right)
$$

Where $G(x, y)$ is complex number with real part $\operatorname{Re}(G(x, y))$ and imaginary part $\operatorname{Im}(G(x, y))$.

Assuming the Gabor parameters $\{\theta, u, \sigma\}$ are given, PalmCode method encodes each pixel in the Gabor filtered image into two bits by the following rules:

$$
\begin{aligned}
& P^{\operatorname{Re}}(x, y)= \begin{cases}0, & \text { if } \operatorname{Re}(G(x, y))<0 ; \\
1, & \text { otherwise. }\end{cases} \\
& P^{\operatorname{Im}}(x, y)= \begin{cases}0, & \text { if } \operatorname{Im}(G(x, y))<0 ; \\
1, & \text { otherwise. }\end{cases}
\end{aligned}
$$

Where $P^{\operatorname{Re}}(x, y)$ and $P^{\operatorname{Im}}(x, y)$ are respectively the encoded real and imaginary binary feature templates.

\subsubsection{Fourier spectrum of PalmCode}

The discrete Fourier transform (DFT) of a function (image) $p(x, y)$ of size $M \times N$ can be given by $P(u, v)=|P(u, v)| e^{\phi(u, v)}$, where $|P(u, v)|=\left[R^{2}(u, v)+\right.$ 
$\left.I^{2}(u, v)\right]^{1 / 2}$ is called the magnitude spectrum of the Fourier transform, and $\phi(u, v)=\tan ^{-1}\left[\frac{I(u, v)}{R(u, v)}\right]$ is called the phase angle or phase spectrum of the transform. $R(u, v)$ and $I(u, v)$ are the real and imaginary parts of $F(u, v)$, respectively.

The Fourier transform has the following translation properties:

If $p(x, y) \Leftrightarrow P(u, v)$,

then

$$
p\left(x-x_{0}, y_{0}\right) \Leftrightarrow P(u, v) e^{-j 2 \pi\left(u x_{0} / M+v y_{0} / N\right)}
$$

and

$$
p(x, y)(-1)^{x+y} \Leftrightarrow P(u-M / 2, v-N / 2) .
$$

Equation (5.5) tells us that a shift in the time domain causes no changes in the magnitude spectrum but only the changes in the phase spectrum of Fourier transform. This property has been widely used to extract translation invariant features in pattern recognition. Equation (5.6) describes that multiplying $p(x, y)$ by $(-1)^{x+y}$ shifts the original of $P(u, v)$ to frequency coordinates $(M / 2, N / 2)$, which is the center of the $M \times N$ area occupied by the 2 -D DFT. This result is based on the variables $u$ and $v$ having values in the range $[0, M-1]$ and $[0, N-1]$, respectively. In a computer implementation these variables will run from $u=1$ to $M$ and $v=1$ to $N$, in which case the actual center of the transform will be at $u=M / 2+1$ and $v=N / 2+1$. This property is usually used when visualizing the 2-D Fourier spectrum.

The DFT has the following conjugate symmetry property:

$$
P(u, v)=P^{*}(-u,-v),
$$

from which it follows that the spectrum is symmetric about the original:

$$
|P(u, v)|=|P(-u,-v)|
$$

Based on the symmetry property, we can only keep half of the Fourier coefficients for image representation.

Figure 5.3 gives an illustration of the proposed Fourier spectral representation scheme. Figure 5.3(a) shows a typical palmprint image from PolyU palmprint 


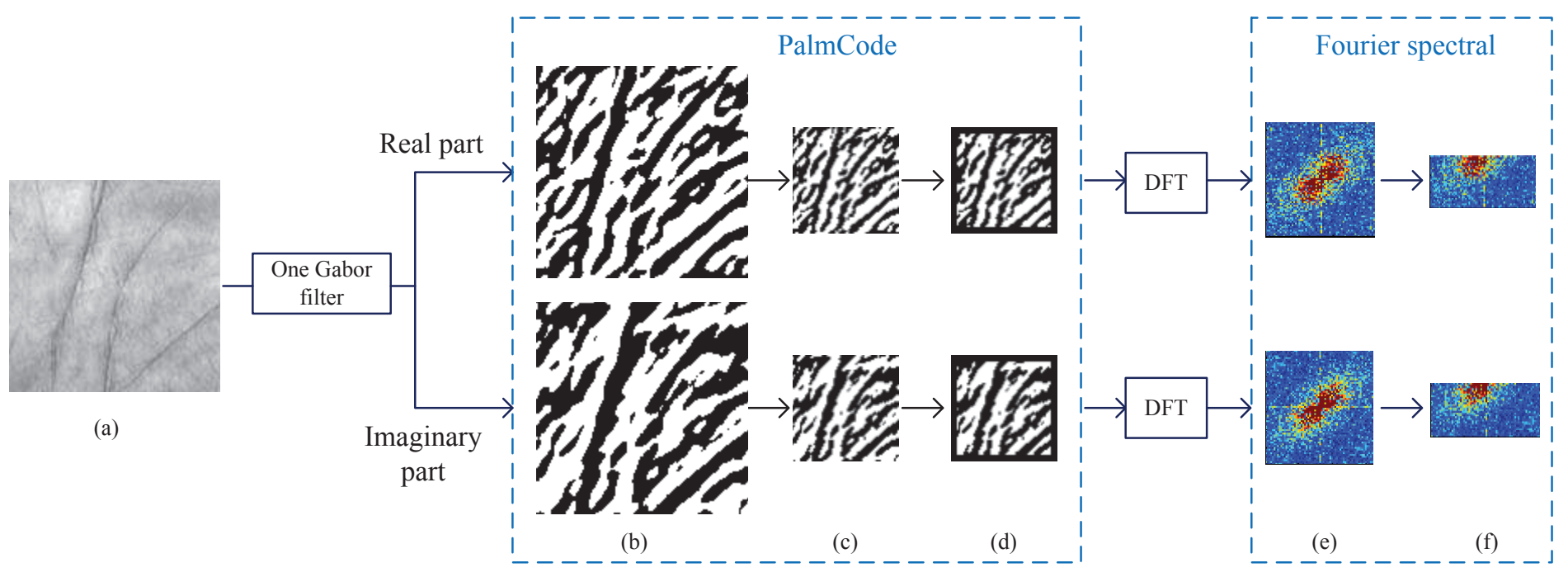

Fig. 5.3: An illustration of the proposed Fourier spectral representation scheme. (a) original image; (b) PalmCode; (c) PalmCode downsampled by ratio of 2:1; (d) Palmcode with the four rows and columns along the code plane edges set to 0; (e) Fourier spectral of (d); (f) half of (e). 
database. Figure 5.3(b) visualizes the original PalmCode feature planes as illustrated in Sec. 5.2.3. For reducing the computational cost, the PalmCode features are down-sampled by the ratio of 2:1 in this study, as can be seen from Fig. 5.3(c). On the edge of the code matrices, there are usually some nonpalmprint features resulted from defective image preprocessing. For reducing their affects, some masks are generally needed in the classical coding based methods $[8,37]$. While in the proposed method, the values of four rows and columns along the code plane edges are changed into zeros for removing the non-palmprint pixels, as Fig. 5.3(d) shows. Figure 5.3(e) shows the Fourier spectral features of PalmCode with the center coordinate $(33,33)$. It is quite obvious that the Fourier spectral matrix is symmetric with respect to the center point. Thus, only half of the spectral values are required for further processing. Figure 5.3(f) shows half of the spectral features, which is the final Fourier spectral representation of our proposed.

\subsubsection{Spectral feature reduction by $(2 \mathrm{D})^{2} \mathrm{PCA}$}

The Fourier spectral feature of PalmCode consists of several real-valued matrices size of $31 \times 64$, as shown in Fig. 5.3(f). The large dimensionality leads to high computational cost, limited matching speed and costly template storage requirement. In order to cope with these problems, we resort to $(2 \mathrm{D})^{2} \mathrm{PCA}$ dimensional reduction technique [48], which is called Modified 2DPCA [100] or horizontal and vertical 2DPCA method [101] in some other papers. The $(2 \mathrm{D})^{2} \mathrm{PCA}$ method not only helps saving the computational cost and providing smaller feature space, but also has a useful invariance property: the transform matrix of horizontal 2DPCA is invariant to any change of image row sequence, and the transform matrix of vertical 2DPCA is invariant to any change of an image column sequence. This invariance property of $(2 \mathrm{D})^{2} \mathrm{PCA}$ helps alleviating the negative effect resulting from image translation [101]. Taking these factors into consideration, $(2 \mathrm{D})^{2} \mathrm{PCA}$ method is sequently carried out on the Fourier spectral feature space.

Given $N$ spectral feature $X_{1}, X_{2}, \ldots, X_{N}$, each spectral $X_{i}(i=1,2, \ldots, N)$ is a $m \times n$ matrix, the goal of horizontal 2DPCA is to find the optimal orthogonal projection axes $U$ so that the projected matrices $Y=\left[Y_{1}, Y_{2}, \ldots, Y_{N}\right]$ achieve 
a maximum total scatter, which can be expressed in the form:

$$
\begin{aligned}
& Y_{i}=X_{i} U, \quad i=1,2, \ldots, N \\
& U=\arg \left(\max \left(\operatorname{tr}\left(S_{Y}\right)\right)\right),
\end{aligned}
$$

where

$$
\begin{aligned}
& \operatorname{tr}\left(S_{Y}\right)=U^{T} S_{X} U \\
& S_{Y}=\frac{1}{N} \sum_{j=1}^{N}\left(Y_{j}-\bar{Y}\right)\left(Y_{j}-\bar{Y}\right)^{T} \\
& S_{X}=\frac{1}{N} \sum_{j=1}^{N}\left(X_{j}-\bar{X}\right)^{T}\left(X_{j}-\bar{X}\right),
\end{aligned}
$$

It follows that $S_{X}$ is a non-negative definite matrix size of $n \times n$. In general, the optimal projection axes $U=\left[u_{1}, u_{2}, \ldots, u_{d}\right]$ are chosen as the orthogonal eigenvectors of $S_{X}$ corresponding to the largest eigenvalues $\lambda_{1}, \lambda_{2}, \ldots, \lambda_{d}$. Thus the projected vectors are $d$-dimensional. Finally, after processed by the horizontal 2DPCA, each spectral matrix is represented as a matrix $Y_{i}$ of dimension $m \times d(d \ll n)$.

After the procedure of horizontal 2DPCA transform, the correlation between row vectors of spectral feature matrices is removed, and the resulted projection matrices are less sensitive to the translation and mirror variations of the spectral row sequences. Further, the vertical 2DPCA transform is needed to remove the correlation between column vectors of the projected matrices $Y_{i}$.

For simplify, the horizontal 2DPCA transform is processed on the transpose of the projected matrices, denoted by $Y_{i}^{T}(i=1,2, \ldots, N)$. Then the corresponding total scatter matrix can be expressed in the form:

$$
\begin{aligned}
C_{Y^{T}} & =\frac{1}{N} \sum_{j=1}^{N}\left(Y_{j}^{T}-\bar{Y}^{T}\right)^{T}\left(Y_{j}^{T}-\bar{Y}^{T}\right) \\
& =\frac{1}{N} \sum_{j=1}^{N}\left(Y_{j}-\bar{Y}\right)\left(Y_{j}-\bar{Y}\right)^{T},
\end{aligned}
$$


which is a non-negative definite matrix size of $m \times m$. Let $V=\left[v_{1}, v_{2}, \ldots, v_{q}\right]$ be the orthogonal eigenvectors of $C_{Y^{T}}$ corresponding to the largest eigenvalues $\gamma_{1}, \gamma_{2}, \ldots, \gamma_{q}(q \ll m)$. By letting $R$ represent the projected matrices of $Y$ after the vertical 2DPCA transform, we have $R^{T}$ is the projected matrices of $Y^{T}$ after the horizontal 2DPCA transform, that is

$$
R^{T}=Y^{T} V
$$

It follows that

$$
R=\left(Y^{T} V\right)^{T}=V^{T} Y=V^{T} X U, \quad R \in \mathbb{R}^{q \times d} .
$$

As can be seen, the dimensionality of the Fourier spectral feature $X$ is reduced from $m \times n$ to $q \times d(d \ll n, q \ll m)$ after the $(2 \mathrm{D})^{2} \mathrm{PCA}$ transform.

As Fig. 5.3 shows, each image generates two spectral feature matrices when one Gabor filter is convolved. Based on the proposed feature extraction framework, we might obtain more robust feature representation for palmprint recognition, if more Gabor filters with different orientations are used. As a matter of fact, we find that the proposed method performs much better when two Gabor filters instead of one are used for spectral feature extraction according to the experimental results. Figure 5.4 illustrates the training steps of the proposed algorithm when two Gabor filters are used, in accordance with which we summarize the proposed palmprint feature extraction algorithm as follows:

Step 1. Input the training palmprint images, and convolve each image with two Gabor filters of different orientation parameters to obtain four PalmCode matrices.

Step 2. Perform the 2-D Fourier transform on each binary PalmCode matrix to get the corresponding spectral feature matrix as described in Fig. 5.3.

Step 3. Perform (2D) $)^{2} \mathrm{PCA}$ transform on these spectral feature matrices got in Step 2 to obtain the corresponding four groups of feature projection matrices $(V 1, U 1),(V 2, U 2),(V 3, U 3),(V 4, U 4)$ as shown in Fig. 5.4.

Step 4. Transform the four Fourier spectral matrices of each training image by the four groups of feature projection matrices respectively to generate four feature matrices as described in Eq. (5.13), denoted by $\{R 1, R 2, R 3, R 4\}$. Save them as the training feature database. 


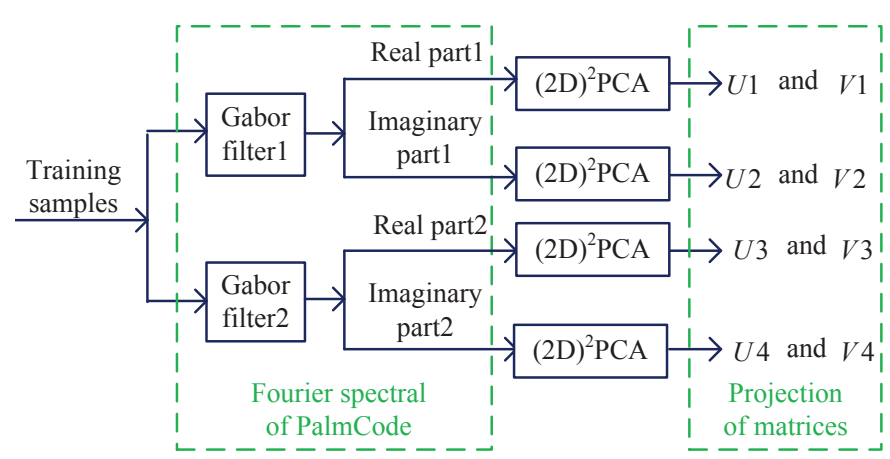

Fig. 5.4: The training steps of the proposed algorithm when two Gabor filters are used. The Fourier spectral of PalmCode is achieved as illustrated in Fig. 5.3.

Step 5. When a query image is captured, the same processing as Steps 1 and 2 is applied, and then transform their Fourier spectral matrices as Step 4 to get the final feature template $\left\{R 1^{\prime}, R 2^{\prime}, R 3^{\prime}, R 4^{\prime}\right\}$.

Step 6. For template matching, the training and test feature matrices are respectively converted into vectors $\{\bar{R} 1, \bar{R} 2, \bar{R} 3, \bar{R} 4\}$ and $\left\{\bar{R} 1^{\prime}, \bar{R} 2^{\prime}, \bar{R} 3^{\prime}, \bar{R} 4^{\prime}\right\}$; Then the dissimilarity is measured by sum of Euclidean distance between each pair of vectors $\bar{R} i$ and $\bar{R} i^{\prime}$, which can be denoted by $\sum_{i=1}^{4} D\left(\bar{R} i, \bar{R} i^{\prime}\right)$.

\subsubsection{Experimental results}

In this section, we evaluate the recognition performance of our proposed method using HongKong Polytechnic University (PolyU) Palmprint Database, which contains 7752 grayscale images in BMP image format. They were captured by CCD camera from 386 different palms and collected in two occasions with different illumination conditions. The interval between the two sessions is about two months. Each person provided around ten palmprint images from the left and right hands respectively. The resolution of original captured images is $384 \times 284$ pixels at 75 dpi. By performing the similar preprocessing approach described in Ref. [12], we cropped the region of interest (ROI) size of $128 \times 128$. Figure 5.5 shows the cropped images from five typical palm samples, in which (a) and (b) denote the samples captured from the same palm at the first and second sessions respectively. 


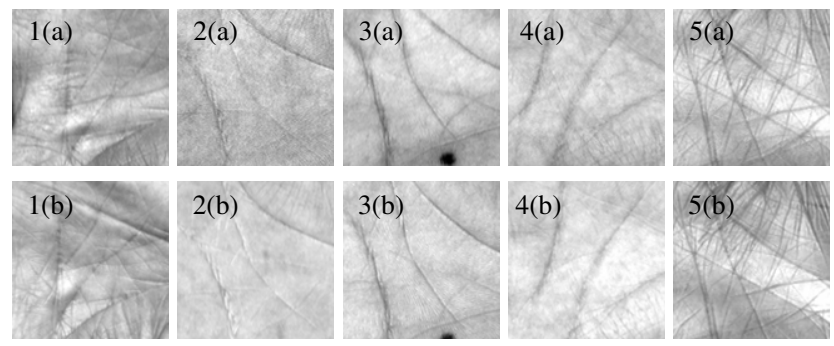

Fig. 5.5: The palmprint images in the Hong Kong PolyU database. (a) Samples in session one; (b) Samples in session two.

\subsubsection{Determination of the parameters}

For the Gabor based methods, there is generally a set of adjustable parameters. For DFT_PalmCode method, Besides Gabor parameters, $d$ and $q$ for $(2 \mathrm{D})^{2} \mathrm{PCA}$ transform are controllable as well. In this group of experiments, we firstly calculate the values $d$ and $q$ by taking $90 \%$ of the total sum of the eigenvalues. Based on the obtained values $d$ and $q$, a series of experiments is carried out to investigate the influence of Gabor orientation parameter and examine how the number of Gabor filters affects the identification rate. Secondly, with the determined optimal Gabor filters, the identification accuracies are investigated as $d$ and $q$ vary.

For each palm, five samples from session one are randomly collected to construct the training set, and all the samples (in total 3863) captured in session two are used for testing. Assuming the system is operated in the identification mode (one-to-many comparison). The average correctness rate (\%) of ten-run identification executions is used to evaluate the identification performance.

By following the experimental results of literature [12], the Gabor parameter $u$ is set to 0.0916 , and $\sigma$ is fixed to 5.6179. In order to determine the optimal number of Gabor filters and their orientation parameters for our proposed method, we test some orientation cases on palmprint identification accuracy. For the applied (2D) $)^{2} \mathrm{PCA}$ transform, values $d$ and $q$ are determined by taking $90 \%$ of the total sum of eigenvalues. When one Gabor filter is used, the considered orientation values are $0^{\circ}, 10^{\circ}, 30^{\circ}, 45^{\circ}, 60^{\circ}, 90^{\circ}, 120^{\circ}, 135^{\circ}, 150^{\circ}$, and $170^{\circ}$, respectively. Besides, the identification rates are also investigated when two and three Gabor filters are used with ten cases of different orientation settings. The tested orientation groups are as follows: $\left\{45^{\circ}, 60^{\circ}\right\}$, $\left\{45^{\circ}, 90^{\circ}\right\},\left\{45^{\circ}, 120^{\circ}\right\},\left\{45^{\circ}, 135^{\circ}\right\},\left\{60^{\circ}, 90^{\circ}\right\},\left\{60^{\circ}, 120^{\circ}\right\},\left\{60^{\circ}, 135^{\circ}\right\}$, $\left\{90^{\circ}, 120^{\circ}\right\},\left\{90^{\circ}, 135^{\circ}\right\},\left\{120^{\circ}, 135^{\circ}\right\},\left\{45^{\circ}, 60^{\circ}, 90^{\circ}\right\},\left\{45^{\circ}, 60^{\circ}, 120^{\circ}\right\},\left\{45^{\circ}\right.$, 


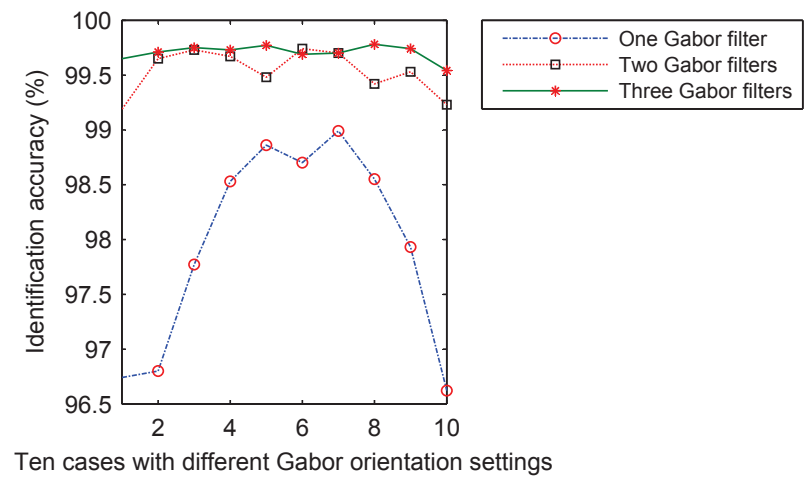

Fig. 5.6: The identification accuracies (\%) versus the ten Gabor orientation setting cases.

$\left.60^{\circ}, 135^{\circ}\right\},\left\{45^{\circ}, 90^{\circ}, 120^{\circ}\right\},\left\{45^{\circ}, 90^{\circ}, 135^{\circ}\right\},\left\{45^{\circ}, 120^{\circ}, 135^{\circ}\right\},\left\{60^{\circ}, 90^{\circ}\right.$, $\left.120^{\circ}\right\},\left\{60^{\circ}, 90^{\circ}, 135^{\circ}\right\},\left\{60^{\circ}, 120^{\circ}, 135^{\circ}\right\},\left\{90^{\circ}, 120^{\circ}, 135^{\circ}\right\}$. Figure 5.6 gives an illustration of the relationship between the identification accuracies and the Gabor orientation parameters. As it shows, the recognition performances of the proposed method improve when the Gabor filter number increases from two to three in most cases. However, the increase of Gabor filter numbers adds to the computational cost in multiple. Based on an overall consideration of the recognition performance and the computational complexity, case 6 with two Gabor filters is determined to be optimum. That is to say, our proposed method achieves the highest identification rate $99.74 \%$ when two Gabor filters with the orientation parameters of $60^{\circ}$ and $120^{\circ}$ respectively are used for the spectral feature extraction on the PolyU palmprint database.

Given the Gabor parameters we got above, the optimal values $d$ and $q$ for $(2 \mathrm{D})^{2} \mathrm{PCA}$ are experimentally investigated by varying $q$ from 10 to 22 and $d$ from 16 to 38. The identification rates corresponding to different $d$ and $q$ are plotted in Fig. 5.7. As can be seen, the best identification rate of our proposed method is close to $99.8 \%$. The feature dimension after $(2 \mathrm{D})^{2} \mathrm{PCA}$ transform is $q \times d$, which is expected to as small as possible while keeping the highest identification rate. Therefore, the best identification performance is obtained when $d=28, q=12$, as can be seen from Fig. 5.7.

It should be pointed out that in other experiments we simply take the sum of the largest $d$ eigenvalues that determine $90 \%$ of the total sum of the eigenvalues when processing the horizontal 2DPCA transform. By the same way, we get the value of $q$ for the vertical 2DPCA transform. 


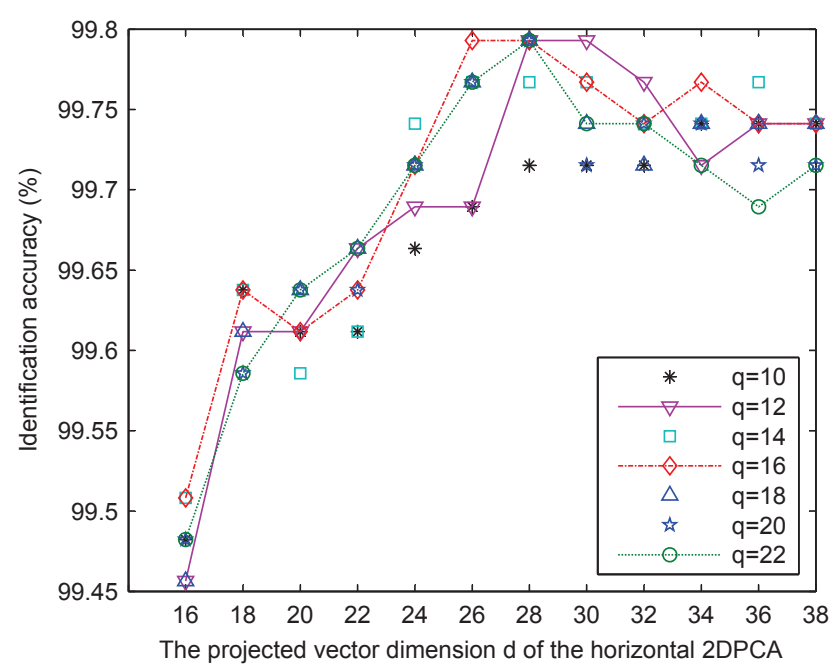

Fig. 5.7: Identification rates (\%) as values $d$ and $q$ vary.

\subsubsection{Comparisons with coding methods}

In this section the palmprint identification and verification performances are investigated when PalmCode is replaced by the CompCode, which I denote by DFT_CompCode. For PalmCode, the used Gabor filter parameters are given by $u=0.0916, \theta=\pi / 4$, and $\sigma=5.6179$; For CompCode, six Gabor filters are used to obtain the competitive code features with the orientation set as $\theta_{j}=\pi j / 6, j=0,1, \ldots, 5 . \quad u$ and $\sigma$ are fixed to 0.0916 and 5.6179 respectively; All the code features mentioned here are down-sampled by ratio of 4:1. For obtaining the matching distance, the code feature matrix needs to be shifted by rows and columns. Here the shifting ranges are set to $0,[-1,1]$ and $[-2,2]$. Time cost for identifying per test sample is recorded by Matlab 7.5 from a personal computer with an Intel Pentium(R) Dual-Core Processor (E5200@2.50GHz) and 2GB RAM configured with Microsoft Windows XP.

When the system works in the identification mode, five samples from session one are randomly collected to construct the training set, and all the samples from session two are used for testing. The average identification rates (\%) of ten-run executions with different methods are listed in Table 5.1. Besides, the time cost of identifying one test sample is also compared under the current data set. When the system operates in the verification mode, all the 7752 samples are used. Each palmprint template is matched with all the other ones to evaluate the receiver operating characteristics (ROC). A genuine matching 
Table 5.1: Comparisons of the average identification accuracy (\%), time cost (s) for identifying per test sample, and the verification EER (\%).

\begin{tabular}{|l|c|c|c|}
\hline Method & $\begin{array}{c}\text { Identification } \\
\text { accuracy (\%) }\end{array}$ & $\begin{array}{c}\text { Time } \\
\text { cost (s) }\end{array}$ & $\begin{array}{c}\text { Verification } \\
\text { EER (\%) }\end{array}$ \\
\hline \hline PalmCode 0 & 75.63 & 0.067 & 29.5 \\
PalmCode [-1,1] & 95.14 & 0.644 & 9.1 \\
PalmCode [-2,2] & 98.27 & 1.66 & 3.1 \\
\hline DFT_PalmCode & 99.74 & 0.123 & 0.7 \\
\hline CompCode 0 & 78.14 & 0.085 & 24.2 \\
CompCode [-1,1] & 96.17 & 0.920 & 7.2 \\
CompCode [-2,2] & 99.11 & 2.398 & 1.9 \\
\hline DFT_CompCode & 99.29 & 0.106 & 0.9 \\
\hline
\end{tabular}

is defined as the matching between the features from the same palm, and otherwise the matching is counted as an impostor. The total number of matches is 30042876 , of which the number of genuine is 74068 and the number of impostor is 29968808 .

Table 5.1 shows the comparisons of the average identification accuracy (\%), time cost (s) for identifying per test sample, and the verification EER (\%). As can be seen, the recognition performance of coding based methods depends badly on the translation extent of code feature plane. Furthermore, the matching speed dramatically drops as the translation range increases. The identification rate of CompCode achieves $99.11 \%$ at the time cost of 2.398 seconds, which is not desirable when the system is operated in the identification mode. DFT_PalmCode outperforms PalmCode significantly in terms of identification performance and verification accuracy because of two reasons. First, DFT_PalmCode fuses more phase information from two Gabor filters with different orientations, instead of one Gabor filtering as PalmCode exploits, which makes the discriminative ability more powerful. Second, using the frequency-domain representation of code features makes it capable of undergoing training process by some learning algorithms, which improves the recognition performance and also reduces the time cost of dissimilarity calculation. By comparing the identification performances of CompCode and DFT_CompCode, it can be seen that the identification accuracy does not improve evidently while the matching speed drops dramatically after executing DFT transform and $(2 \mathrm{D})^{2} \mathrm{PCA}$ on the code features. This may be because the processes of DFT transform and $(2 \mathrm{D})^{2} \mathrm{PCA}$ do not extract more discriminative information from the image, but their resulting real-valued feature templates cost less than the logical-valued code feature planes. From Table 5.1 it can be also seen that lower EER is achieved by operating the DFT and $(2 \mathrm{D})^{2} \mathrm{PCA}$ 
(a)

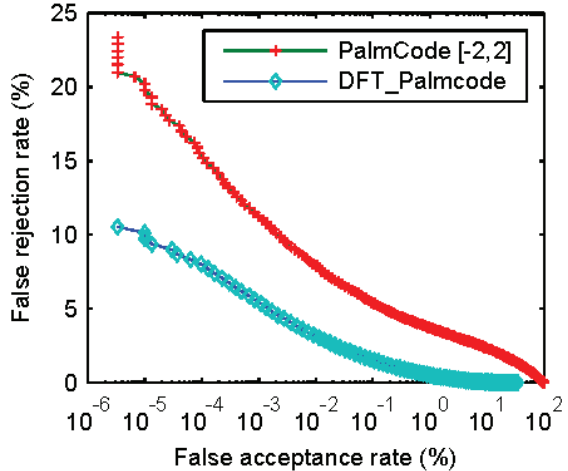

(b)

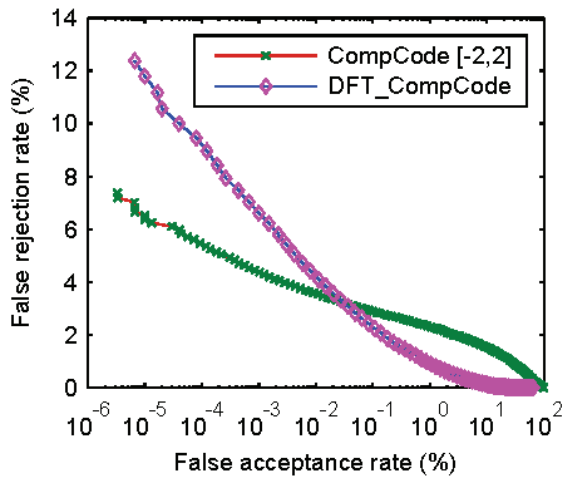

Fig. 5.8: Comparison of the verification accuracies (\%) (FAR versus FRR). For the coding based methods, the code template is shifted horizontally and vertically for multiple times within a range $[-2,2]$.

transform on the code features. Since the matching score measurements for coding based methods and the proposed feature representation framework are totally different, we plot their ROC curves for comparisons further. From Fig. 5.8(a) and (b), we can see that DFT_CompCode does not improve the verification accuracy of CompCode absolutely though it does achieve lower EER. However, DFT_PalmCode does perform much better than PalmCode.

\subsubsection{Comparisons with other methods}

Gabor filtering based methods have been widely investigated in various image representation field. Besides coding based methods, there are two other classes: First, Gabor coefficients based statistical learning methods, which 


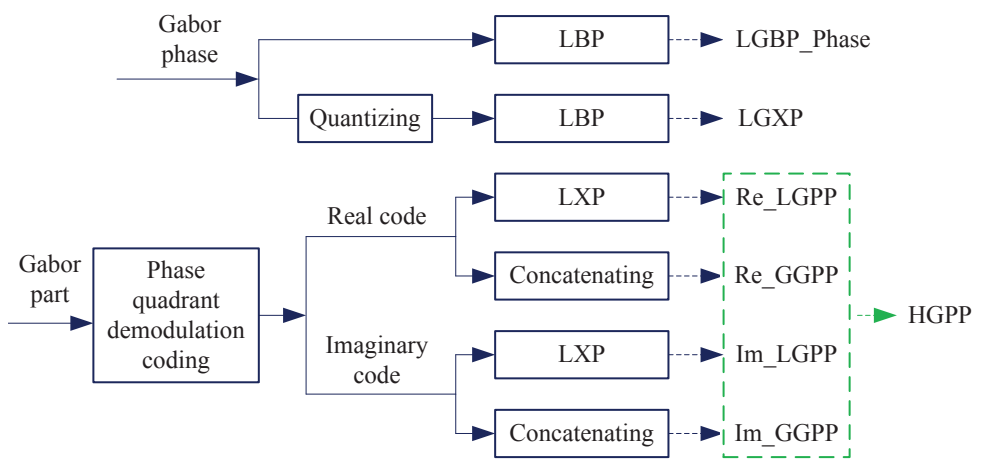

Fig. 5.9: An illustration of various local pattern operators compared in this paper. (LBP local binary pattern, LXP - local XOR pattern)

make the statistical analysis of the Gabor magnitude coefficients [48-50,55]; Second, local Gabor pattern histogram methods [70,79-81], which fuse the Gabor coefficients with different local pattern operators to achieve histogram sequences as descriptors. In this section, comparisons with some of them are evaluated on the palmprint recognition performance. Note that for principal component analysis (PCA) and linear discriminant analysis (LDA) involved in these methods, we take the sum of the first largest eigenvalues that determine $90 \%$ of the total sum of the eigenvalues for fair comparisons.

GM_(2D $)^{2}$ PCA [48], as a representative method of Gabor coefficients based statistical learning methods, is compared in this group of experiments. A bank of five-scale and eight-directional filters (in total 40 filters) is firstly used to derive a Gabor feature space of high dimensionality and then two steps of 2DPCA as described in Sec. 5.2.5 are carried out to reduce the dimension. The Gabor parameters are set as follows: $\theta_{j}=\pi j / 8, j=0,1, \ldots, 7$, $u_{v}=0.2592 / \sqrt{2^{v}}, v=0,1, \ldots, 4, \sigma=5.6179$. For GM_(2D) ${ }^{2}$ PCA method, the Gabor coefficients are down-sampled by the ratio of 4:1. Otherwise, the Gabor feature dimensionality will be too high to be processed further in our experimental system.

By encoding Gabor magnitude and phase coefficients via local binary pattern (LBP) operator, LGBP_Mag [70] and LGBP_Pha [79] have been proposed respectively. By using the local XOR pattern (LXP) operator to encode the real and imaginary parts of Gabor complex response, local Gabor phase pattern (LGPP) has been proposed [80], denoted by Re_LGPP and Im_LGPP respectively. Different from LGPP, GGPP has been proposed to represent orientation patterns, which forms one eight-bit binary string to represent each pixel by concatenating the real or imaginary quadrant-bit codes of different 
Table 5.2: Comparisons of the average identification accuracy (\%) and feature length among different methods. For evaluating the identification performance, the training sample number per class ranges from two to five. The average identification rate is calculated over ten-run executions with various training and testing sets.

\begin{tabular}{|l|c|c|c|c|c|c|c|}
\hline \multirow{2}{*}{ Method } & \multicolumn{3}{|c|}{ Identification rate $(\%)$} & \multirow{2}{*}{$\begin{array}{c}\text { Gabor } \\
\text { number }\end{array}$} & $\begin{array}{c}\text { Feature length } \\
\text { before reduction }\end{array}$ & $\begin{array}{c}\text { Final } \\
\text { feature length }\end{array}$ \\
\cline { 2 - 4 } & 5 & 4 & 3 & 2 & 2 & 7936 & 2878 \\
DFT_PalmCode & 99.74 & 99.67 & 99.55 & 99.24 & 40960 & 13680 \\
GM_(2D) ${ }^{2}$ PCA & 81.42 & 80.34 & 77.65 & 74.84 & 40 & 40960 & 1540 \\
Re_LGPP & 97.10 & 96.87 & 96.20 & 94.18 & 40 & 40960 & 1540 \\
Im_LGPP & 97.22 & 97.10 & 96.56 & 95.48 & 40 & 92160 & 1540 \\
HGPP & 98.40 & 98.11 & 97.93 & 97.82 & 40 & 40960 & 1540 \\
LGBP_Phase & 97.75 & 97.57 & 97.08 & 95.92 & 40 & 40960 & 1540 \\
LGXP & 98.24 & 97.97 & 97.42 & 97.15 & 40 & & \\
\hline
\end{tabular}


orientations for a given frequency (denoted by Re_GGPP and Im_GGPP). In addition, Xie [81] proposed local Gabor XOR pattern (LGXP) by quantizing the Gabor phase in each local region firstly, and then encoding the quantized phases by LXP operator. Figure 5.9 gives an illustration of different local Gabor pattern operators. For fair comparisons, the Gabor parameters used in all the mentioned local Gabor pattern methods are the same as those set in GM_(2D $)^{2}$ PCA method.

We assume that the system works in the identification mode, samples from session one are used for training, and all the samples from session two for test. Different training sets are constructed respectively with the sample numbers of each palmprint class ranging from two to five. The training samples are randomly selected and ten-run executions are averaged to evaluate the identification rate (\%). Besides, the resulting feature size is also investigated for evaluating the computational complexity.

Table 5.2 lists the comparisons of palmprint identification performance. As it shows, the proposed method achieves the highest identification rate by a large margin. Even under the condition of less training samples, the identification accuracy of DFT_PalmCode method reaches up to $99.24 \%$. Different from the other Gabor based methods, which extract multi-scale and multi-directional features using 40 Gabor filters, DFT_PalmCode uses only two Gabor filters. Since the Gabor filtering is time-costing, less used filters leads to the advantages of higher execution speed and lower storage requirement. Besides, all the local Gabor pattern methods generate a histogram feature space with high dimensionality. As the number of resulting sub-blocks increases, the histogram dimension will extend in multiple. Given the sub-block number be $N$, and the image class number $C$, then the final feature vector length will be $N \times(C-1)$. As Table 5.2 shows, $N \times(C-1)=4 \times(386-1)=1540$ is the final feature size of the mentioned local Gabor pattern methods in this group of experiments, while the final feature length of DFT_PalmCode is 2878, which is larger but still comparable.

\subsubsection{Conclusion and future work}

In this paper, we have presented a new feature extraction method for palmprint recognition. The Gabor phase information is firstly encoded into binary code features, which are then transformed into Fourier magnitude spectrum (denoted by DFT_PalmCode) as palmprint descriptor. The spectral features 
are further processed by the horizontal and vertical 2DPCA transform for palmprint recognition. Experimental results demonstrate its high efficiency under both verification and identification system mode.

The main contributions and conclusions of this paper are as follows: (1) The proposed method is related to the original PalmCode approach, but they are quite different. First, the real-valued spectral features are achieved for palmprint recognition instead of the binary code matrices, which not only keep the high discriminative ability of PalmCode features, but also can be processed by some learning algorithms to further improve the recognition performance. Second, due to the translational invariance property of Fourier and $(2 \mathrm{D})^{2} \mathrm{PCA}$ transform, DFT_PalmCode method overcomes the weakness of pixel-to-pixel matching strategy, which is widely applied by the coding based methods as well as PalmCode. Third, under the proposed feature extraction framework, more than one Gabor filters can be utilized to fuse phase information, which improves the recognition accuracy evidently compared with PalmCode. (2) The contrast experimental results between another coding method and our method demonstrate that the proposed feature extraction framework can be used on other code-plane based features for increasing identification speed as well as PalmCode. (3) Compared with other state-of-the-art Gabor phase based methods which generally use 40 Gabor filters, the proposed method greatly outperforms in terms of recognition accuracy, computational complexity and storage requirement by using only two Gabor filters.

\subsection{Chapter conclusion}

In this chapter, a real-valued palmprint representation is proposed based on a classical coding-based palmprint recognition method (palm code). The original palm code is robust to image illumination changes, but sensitive to image translation. Accordingly, the proposed method resorts to the Fourier transform and the $(2 \mathrm{D})^{2} \mathrm{PCA}$ transform to further process the palm code. Due to their translation invariance property, the resulting new representation is robust to image translation, as well as to image illumination variations. With regards to the research objective which we formulate in Section 1.3.3, this chapter aims to a real-valued feature representation for an unprotected recognition system. The proposed representation performs better than the palm code method by higher recognition accuracy and faster matching speed. In addition, it significantly outperforms the typical Gabor phase-based methods in terms of recognition accuracy and feature size. 
$\sqrt{\Gamma_{\text {conet }}} 6$

\section{Binary representation on one-bit}

\section{quantization}

\subsection{Chapter introduction}

PURPOSE. In this chapter, a binary palmprint representation is introduced, which aims for a template protection system based on the helper data scheme (HDS). In the previous chapters, several real-valued representations have been presented for the unprotected recognition system. The recognition accuracy is the most concerned system performance. In contrast, this chapter investigates the method of obtaining a fixed-length binary string for palmprint representation. We propose to do the one-bit quantization on some real-valued features. Based on the quantization output, the most reliable bits are selected as the final binary representation. The achieved binary string is required to be discriminative and reliable. The discriminative ability is measured by false rejection rate and false acceptance rate (FRR/FAR). And the reliability is measured by bit error rate (BER) of the genuine binary template matching.

CONTENTS. The proposed binary feature extraction method involves real- 


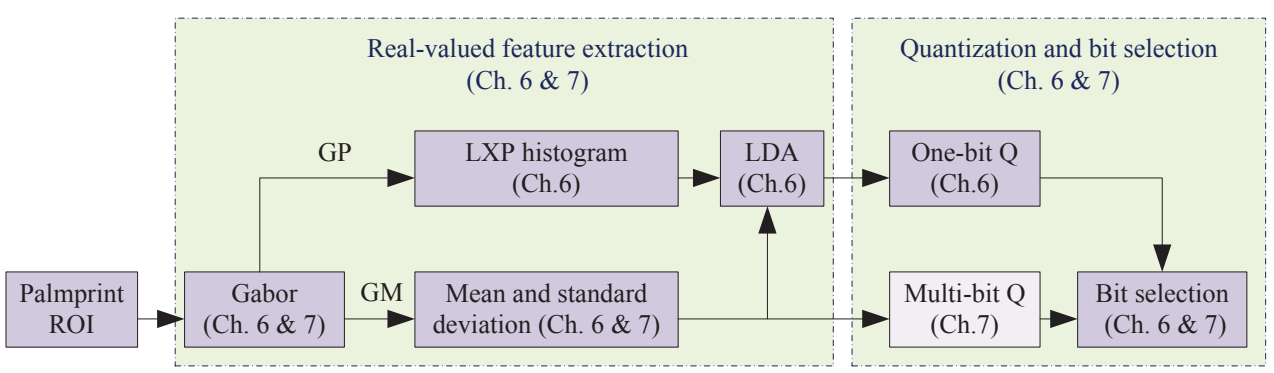

Fig. 6.1: Block diagram of our designed system, highlighting the context of Chapter 6 and its referred blocks.

valued feature extraction, bit quantization and selection. In Section 6.2.3 the Gabor filtered real-valued feature extraction method is presented, which exploits both of the Gabor magnitude (GM) and Gabor phase (GP) information. For GP, the region-based local pattern histogram statistical features are extracted. And for GM, the region-based mean and standard deviation statistical features are extracted. In order to generate the bits of identically and independently distribution, the linear discriminant analysis (LDA) is adopted, which contributes to the good verification accuracy as well. In Section 6.2.4, the one-bit quantization and bit selection approach is introduced. The quantization is based on a threshold. The selection depends on the absolute difference between the real value and the threshold. The number of selected bits is experimentally determined. Experiments are executed over the HongKong PolyU palmprint database. The comparison between the proposed method and one of the coding-based methods for unprotected verification system is given. In the context of system diagram, the content of this chapter and its referred blocks are highlighted in Figure 6.1.

PUBLICATION(s). The content of Section 6.2 of this chapter has been published in [102]. 


\subsection{Binary features on Gabor filtering and one-bit}

\section{quantization}

\subsubsection{Abstract}

The biometric template protection system requires a high-quality biometric channel and a well-designed error correction code (ECC). Due to the intra-class variations of biometric data, an efficient fixed-length binary feature extractor is required to provide a high-quality biometric channel so that the system is robust and accurate, and to allow a secret key to be combined for security. In this paper we present a binary palmprint feature extraction method to achieve a robust biometric channel for template protection system. The real-valued texture statistical features are firstly extracted based on Gabor magnitude and phase responses. Then an approach of one-bit quantization and reliable bit selection is presented. Experimental results on the HongKong PloyU Palmprint database verify the efficiency of our method which achieves low verification error rate by a robust palmprint binary representation of low bit error rate.

\subsubsection{Introduction}

It has been widely known that the typical biometrics system encounters some security and privacy problems such as identity fraud, limited-renewability, cross-matching, and leaking sensitive personal information [3]. Biometric template protection system, as a countermeasure to these security and privacy threats, has become an important issue, which requires that biometric data is firstly quantized into a fixed-length binary string as template. For typical (unprotected) palmprint verification systems, there have been many feature representation approaches reported achieving high verification accuracy. Among them there are some coding based methods which generate binary features $[12,41]$. However, most of them require a template registration during the matching stage, which might be not allowed for template protection system. Further, most of the current reported binary representations give the bit error rates as high as $40 \%$ from the genuine matching, which are too noisy to be corrected by the current error correcting coding schemes [103,104].

In this paper, we present a method of binary feature extraction from palm- 


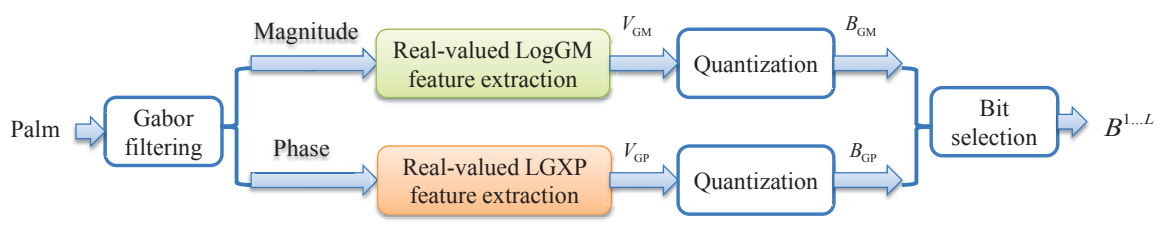

Fig. 6.2: Flow chart of the proposed method.

print image, aiming to achieve robust biometric channel, $i$. e. low bit error rate (BER) for matching channel, and low verification error rate, which we indicate by false rejection rate (FRR), false acceptance rate (FAR) and equal error rate (EER). The robust binary representation gives a solution of combing the typical biometric verification and the template protection. Besides, a fixed-length binary representation also has additional advantages such as small template storage and high matching speed. Since the over-complete information of Gabor filtering responses contributes to the discriminating ability, our method chooses to filter the palmprint image by a group of two-dimensional Gabor functions firstly [12]. Figure 6.2 shows the flow chart of our proposed binary feature extraction method. As can be seen from it, instead of employing the filtered Gabor magnitude (GM) and Gabor phase (GP) responses directly which are generally high-dimensional, we extract the statistic features from them respectively for real-valued representation (denoted by $V_{G M}$ and $V_{G P}$ ), then based on which the one-bit quantization and reliable bits selection are subsequently processed. Finally a binary sequence (denoted by $B^{1 \ldots L}$ ) length of $L$ is achieved as palmprint template for storage and matching. From GM information the global statistical features are extracted which we denote by LogGM [55], while from GP information the local statistical features are extracted after the local XOR pattern (LXP) operating which we denote by LxpGP [81]. After quantizing the global and local statistical features respectively, we fuse the obtained binary bits together and then from them some reliable bits are selected to construct the final binary Gabor statistical features. For verification the Hamming distance is employed as the dissimilarity measurement.

The main contributions of this paper are: a highly efficient scheme is proposed to extract the binary features from the Gabor filtering responses by considering the fusion of the local and global texture statistical information, which provides a high-quality biometric channel achieving low bit error rate for the genuine template matching and the corresponding low verification error rate so as to give a solution of combining the typical palmprint verification system and template protection scheme. 
In this paper, we will firstly present the scheme of real-valued Gabor texture statistical representation in Sec. 6.2.3, which includes the statistical analysis of Gabor magnitude and phase responses respectively. Next, the one-bit quantization and reliable bit selection algorithm will be introduced in Sec. 6.2.4. The experimental results will be given in Sec. 6.2.5. Finally, it is the conclusion.

\subsubsection{Gabor statistical feature extraction}

In order to represent a palmprint by a binary string for template protection, we need to firstly extract some real-valued features from it. Since the Gabor filtered representation can provide the optimal localization of image details, we choose a group of Gabor functions to perform a joint spatial-frequency multi-channel transform on the palmprint image, which can be expressed as following [12]:

$$
\begin{aligned}
g_{m, n}(x, y) & =\frac{1}{2 \pi \sigma^{2}} \exp \left\{\frac{-\left(x^{2}+y^{2}\right)}{2 \sigma^{2}}\right\} \\
& \times \exp \left\{2 \pi i\left(u_{m} x \cos \theta_{n}+u_{m} y \sin \theta_{n}\right)\right\} .
\end{aligned}
$$

$u_{m}$ is the frequency of sinusoidal wave along directional $\theta_{n}$ from $x$-axis, and $\sigma$ specifies the Gaussian envelope along $x$ and $y$ axes, which determines the bandwidth of the Gabor filter. Each Gabor function $g_{m, n}(x, y)$ with the parameters $\left(u_{m}, \theta_{n}, \sigma\right)$ is commonly transformed into a discrete Gabor filter and its direct current is turned to zero, which can be denoted by $\tilde{g}_{m, n}(x, y)$. Given an image $I(x, y)$, its Gabor-filtered images can be defined as follow: $J_{m, n}(x, y)=\tilde{g}_{m, n}(x, y) * I(x, y)$, where $J_{m, n}(x, y)$ is a complex number. The Gabor magnitude and phase angle responses can be respectively denoted by $G M_{m, n}(x, y)=\left\|J_{m, n}(x, y)\right\|$, and $\phi_{m, n}(x, y)=\arctan \left(J_{m, n}(x, y)\right)$, where $\|\bullet\|$ denotes the modulus operator of a complex number. To alleviate the sensitivity of Gabor phase to the varying positions, we transform $\phi_{m, n}(x, y)$ into four different ranges as the Gabor phase (GP) information $G_{m, n}(x, y)$ by the following expression:

$$
G P_{m, n}(x, y)=p, \text { if } 90 *(p-1) \leq \phi_{m, n}(x, y)<90 * p ; \quad p \in\{1,2,3,4\}
$$




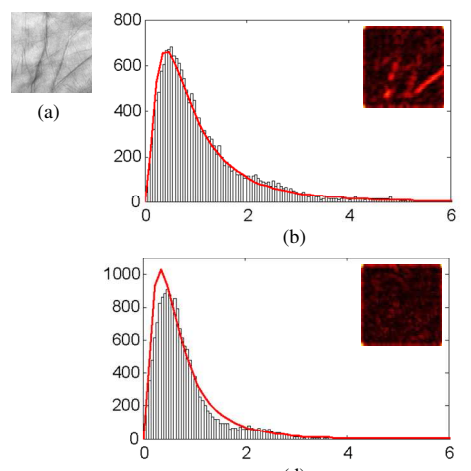

(d)

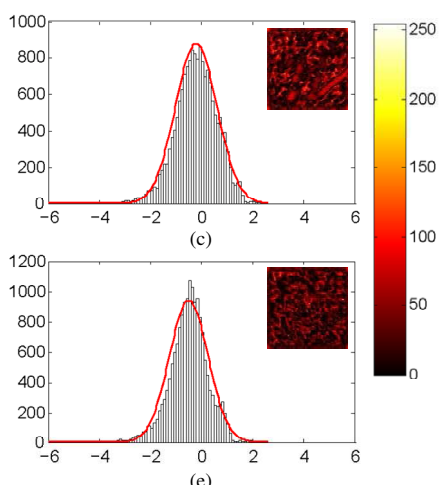

Fig. 6.3: Examples of histogram fitting. (a) The original palmprint image. (b) and (d) hist Gabor magnitudes from two different Gabor parameters. (c) shows the logarithmic transform of (b). (e) is the logarithmic transform of (d).

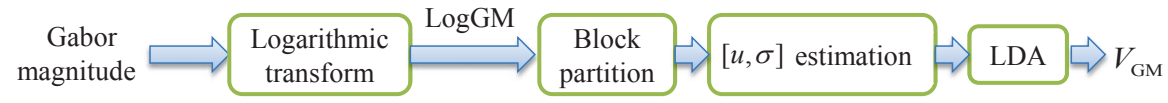

Fig. 6.4: Flow chart of real-valued LogGM feature extraction.

$G M_{m, n}(x, y)$ and $G P_{m, n}(x, y)$ will be respectively further processed to construct the real-valued statistical features.

By investigating the histogram distribution of each GM, it has been found that the lognormal densities fit the GMs very well, and the sub-blocks of each GM are also close to lognormal distribution [55]. Figure 6.3 gives an example of GM histogram fitting. After the lognormal transformation of each GM, some Gaussian distributions are obtained, which can be expressed as $\log G M_{m, n}(x, y)=\log \left(G M_{m, n}(x, y)\right)$. Since a Gaussian sequence can be represented specifically by its mean $\nu$ and standard deviation $\rho$, the palmprint feature representation can be constructed by these Gaussian parameters. Assuming the Gabor filter bank has $E$ scales and $F$ orientations, and each GM is partitioned into $A$ sub-blocks, we will get $(E \times F \times A)$ pairs of $\nu$ and $\rho$ values. By concatenating them together, the final real-valued feature vector can be formulated as $\left[\nu_{1} \ldots \nu_{(E \times F \times A)}, \rho_{1} \ldots \rho_{(E \times F \times A)}\right]$. Following the experimental results in Ref. [55], the best verification performance is achieved when $E=5, F=8, A=21$. To obtain more discriminating feature components from which we expect to extract bits, the linear discriminant analysis (LDA) projection is applied to get the final real-valued representation $V_{G M}$ of GM, the process of which we denote by real-valued LogGM feature extraction as Fig. 6.4 illustrates. 


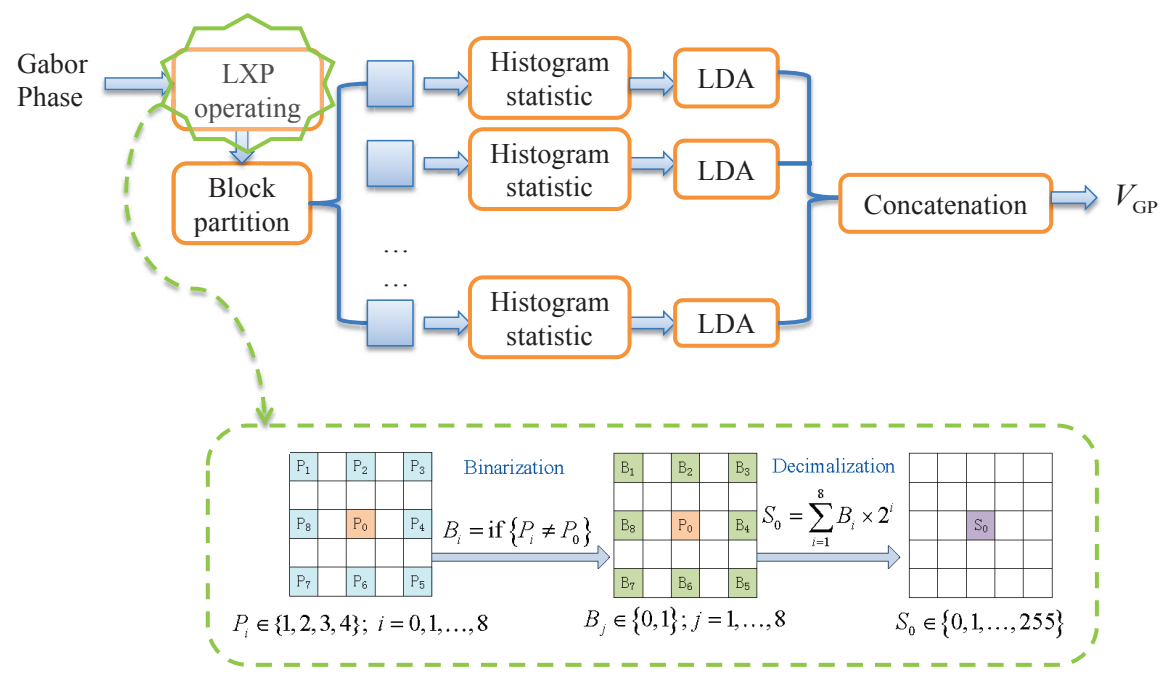

Fig. 6.5: Flow chart of real-valued LxpGP feature extraction.

To extract some statistical features from GP, we firstly encode $G P_{m, n}(x, y)$ by local XOR pattern (LXP) operator [81], which is expressed as $\operatorname{Lxp} G P_{m, n}(x, y)=$ $\operatorname{LXP}\left(G P_{m, n}(x, y)\right)$. As shown in Fig. 6.5, for each pixel $P_{0}$, its eight neighborhoods with one pixel interval $P_{i} \in\{1,2,3,4\}, i=\{0,1, \ldots 8\}$ need to be encoded into $B_{i} \in\{0,1\}, i=0,1, \ldots 8$ by computing $B_{i}=$ if $\left\{P_{i} \neq P_{0}\right\}$. Then $P_{0}$ is mapped into a decimal number $S_{0} \in\{0,1, \ldots 255\}$ by calculating $S_{0}=\sum_{i=1}^{8} B_{i} \times 2^{i}$. Finally all the mapped $S_{0}$ forms $\operatorname{Lxp} G P_{m, n}(x, y)$. Assuming the Gabor filter bank has $E$ scales and $F$ orientations, and each GM is partitioned into $D$ sub-blocks, the histograms of each sub-block at all the scales and orientations are concatenated. Then $D$ histograms of $E \times F \times 256$ bins will be obtained. LDA is applied for each histogram respectively. In this paper, we set $E=5, F=8, D=5$ by taking into account the computational complexity and the performance. Finally the features from the $D$ LDA modulus are concatenated into a vector $V_{G P}$ as the real-valued representation of GP information, the flow chart of which is shown in Fig. 6.5.

\subsubsection{One-bit quantization and reliable bits selection}

Feature quantization and bits selection procedures strongly affect the verification performance of the template protection system. Based on the real-valued features described in Sec. 6.2.3, we introduce our proposed bit quantization 


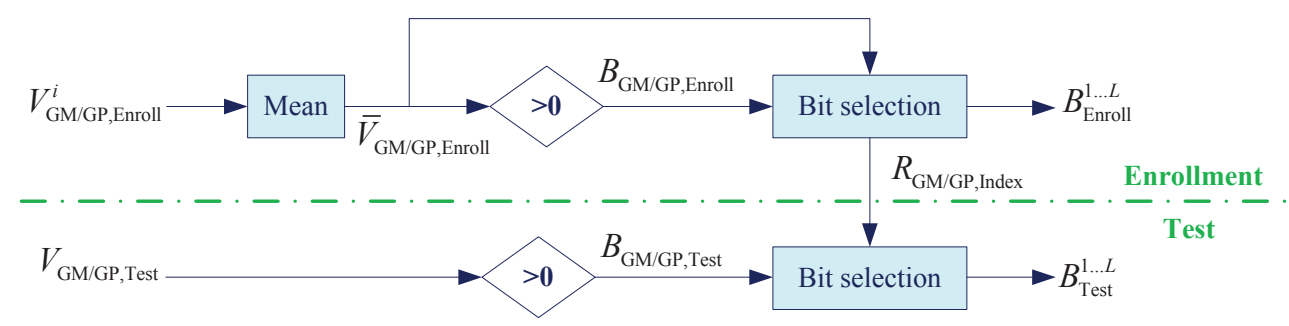

Fig. 6.6: One-bit quantization and reliable bits selection.

and selection method in this section, which is illustrated in Fig. 6.6. Since $V_{G M}$ and $V_{G P}$ are processed in the same way, here we take $V_{G M}$ as an illustration of the main steps as follows:

(1) Compute the averaged real-valued feature from the enrolled $N$ samples of one palm;

Assuming $V_{t}$ palms are captured for training and $N$ samples from one palm are enrolled, we will get a feature set $\left\{V_{\mathrm{GM}, \text { Enroll }}^{i}, i=1 \ldots N\right\}$ to represent each palm after the LDA projection. The averaged feature vector for each palm needs to be computed as expressed following:

$$
\bar{V}_{\mathrm{GM}, \text { Enroll }}=\frac{1}{N} \sum_{i=1}^{N} V_{\mathrm{GM}, \text { Enroll }}^{i, j}, j=1 \ldots\left(V_{t}-1\right)
$$

(2) Quantize the real-valued features by a threshold;

Here the threshold is set to 0 . Each component of the real-valued features $\bar{V}_{\mathrm{GM}, \text { Enroll }}$ is quantized into one bit $B_{\mathrm{GM}, \text { Enroll }}^{j}, j=1 \ldots V_{t}-1$ which can be expressed as following:

$$
\left(B_{\mathrm{GM}, \text { Enroll }}^{j} \mid j=1 \ldots V_{t}-1\right)= \begin{cases}1, & \bar{V}_{\mathrm{GM}, \text { Enroll }}^{j} \geq 0 \\ 0, & \text { otherwise }\end{cases}
$$

By the same process, $V_{\mathrm{GM}, \text { Test }}$ from each test sample is quantized into a binary string $B_{\mathrm{GM}, \text { Test }}$.

(3) Select some reliable bits as the final binary feature template. 


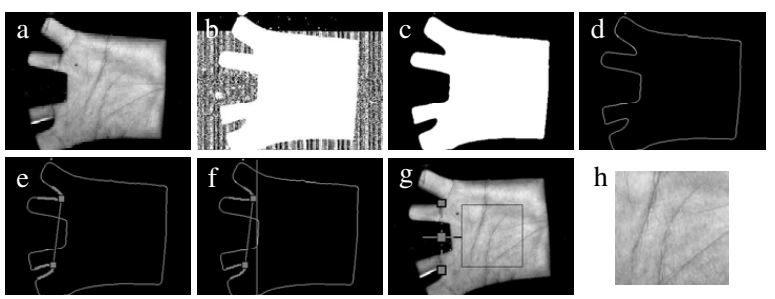

Fig. 6.7: (a) A typical palmprint image from HongKong PolyU Palmprint Database; (b)-(h) show our used registration and region-crop method.

During the enrollment phase, we assume that the larger $\left|\bar{V}_{\mathrm{GM}, \text { Enroll }}^{j}\right|$, the more reliable of its corresponding bit $B_{\mathrm{GM}, \text { Enroll }}^{j}$. In the meanwhile, the positions of the most reliable bits need to be recorded as a index vector $R_{\mathrm{GM}, \text { Enroll }}$. For a test sample, the bits are selected by following the position index of $R_{\mathrm{GM}, \mathrm{En} \text { roll }}$. Assuming $L$ bits are selected then our proposed binary Gabor statistical features are $B_{\text {Enroll }}^{1 \ldots L}$ for enrollment palmprint and $B_{\text {Test }}^{1 \ldots L}$ for test palmprint respectively.

\subsubsection{Experimental results}

The HongKong Polytechnic University (PolyU) palmprint database is used to test our proposed method [6]. They were captured by a CCD camera from 386 different palms and collected in two sessions with two different illumination conditions. There are 3889 images in session one and 3863 palms in session two respectively. Because there is one palm which has only one sample captured in session two, we use the other 385 palms for our experiments. The resolution of original captured images is $384 \times 284$ pixels at 75 dpi. By preprocessing each captured image (as shown in Fig. 6.7), the central region size of $128 \times 128$ is used for feature extraction.

Experiment I: Since the verification performance is heavily depended on the number of the selected bits, we test our algorithm by varying the bits number from 20 to 400. For this experiment, the images in session one are used, which are randomly split as the training set (185 palms) and the evaluation set (the remaining 200 palms for enrollment and test), which can be referred as training-evaluation-set split. For training, all the samples are used. For enrollment, five samples are randomly selected and the remaining ones for test. The training-evaluation-set split is performed six times. For matching genuine pairs, we test all the samples in the test set. For matching imposter 


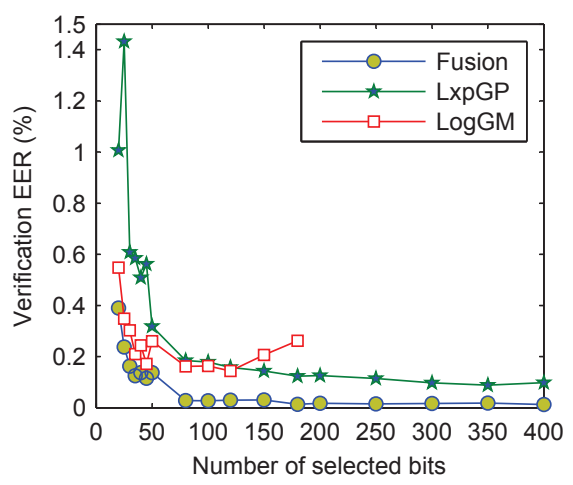

(a)

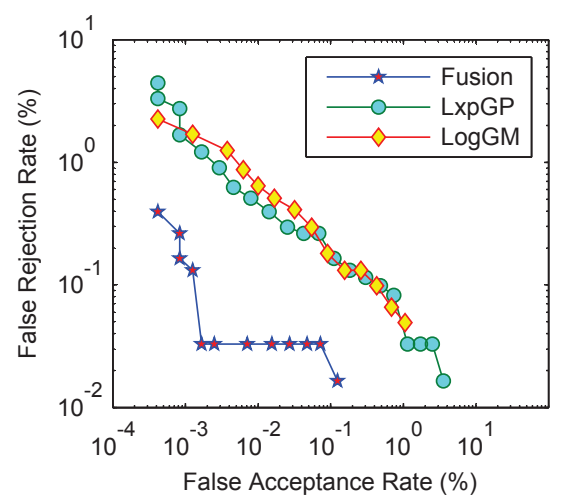

(b)

Fig. 6.8: Verification performance. (a) Verification EER (\%) comparison when the number of selected bits varies from 20 to 400; (b) ROC curves when the number of selected bits is set to 120 .

pairs, the first sample of each palm in the test set is chosen for all the possible imposter combinations. Thus, we have totally 6082 genuine scores and 238,800 imposter scores.

Figure 6.8(a) (Left) plots the comparison of the verification equal error rate (EER) among the methods of LogGM (only GM information is used), LxpGP (only GP information is used), Fusion (the proposed method by fusing GM and GP information). As can be seen from it, the EER decreases as the bits number increases. However, since more bits lead to be more noisy string, we determine 120 as the optimal bits number for the proposed fusion method. Figure 6.8(b) (Right) plots the comparison of ROC curves. As can be seen, the verification performance can be greatly improved by fusing the bits generated from GM and GP information respectively. Figure 6.9 shows the percentage distributions of bit error rate (BER) for genuine and imposter matching respectively by the proposed method. As we can see that the proposed method is able to achieve good verification performance by adjusting the BER threshold of error correcting coding (ECC) module to around 30\% assuming the system works under the template protection framework.

Experiment II: Here the proposed method is compared with the binary co-occurrence vector (BOCV) method on the verification error rate and the corresponding BER threshold which is depended on the used ECC module $[41,104]$. For this experiment, the samples for training (185 palms) are from session one while the ones for evaluation (200 palms) from session two. Other 


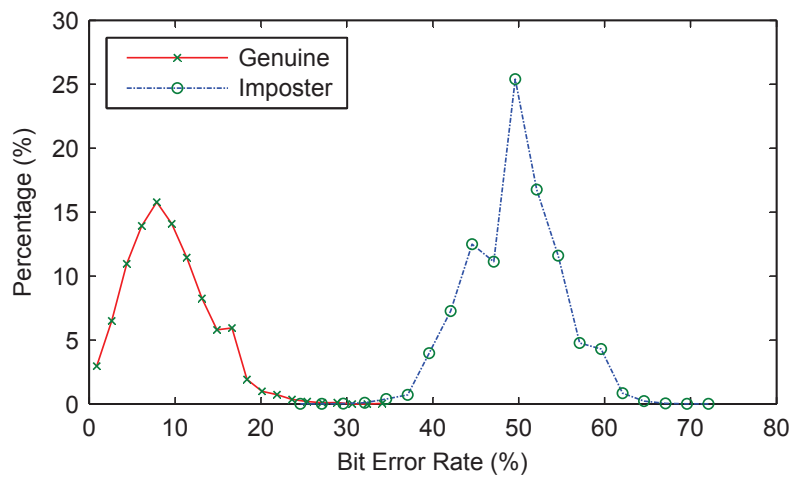

Fig. 6.9: Percentage distribution of Bit Error Rate (\%) for genuine and imposter matching respectively by the proposed method.

protocols are the same as those in experimental I:. For the BOCV method, no training procedure is needed. Here we set the evaluation protocol the same as that for ours. In total, we have 6013 genuine scores and 236,412 imposter scores. BOCV requires to shift the whole image by several pixel horizontally and vertically and then matches multiple times to get the final matching score. Here each bits plane is down-sampled into a binary matrix size of $32 \times 32$. The range of shift is considered as $[-2,2]$.

Figure 6.10 plots the comparison of verification error rate which is indicated by false acceptance rate (FAR) and false rejection rate (FRR) when adjust the BER threshold of ECC module. As can be seen from it, the proposed method greatly outperforms the coding based method BOCV when the applied ECC module has an error correcting capability of lower than $25 \%$. Besides, for the proposed method there is no need to shift the feature templates multiple times for the final matching score, which is not only time-consuming, but also challenges the combination of palmprint verification and template protection system.

\subsubsection{Conclusion and discussion}

In this paper, we present a binary feature extraction method for palmprint verification under consideration of feature template protection which requires a robust biometric channel. Experimental results demonstrate that fusion of the global and local statistical features extracted from Gabor magnitude and phase responses respectively outperforms that of global or local features represented 


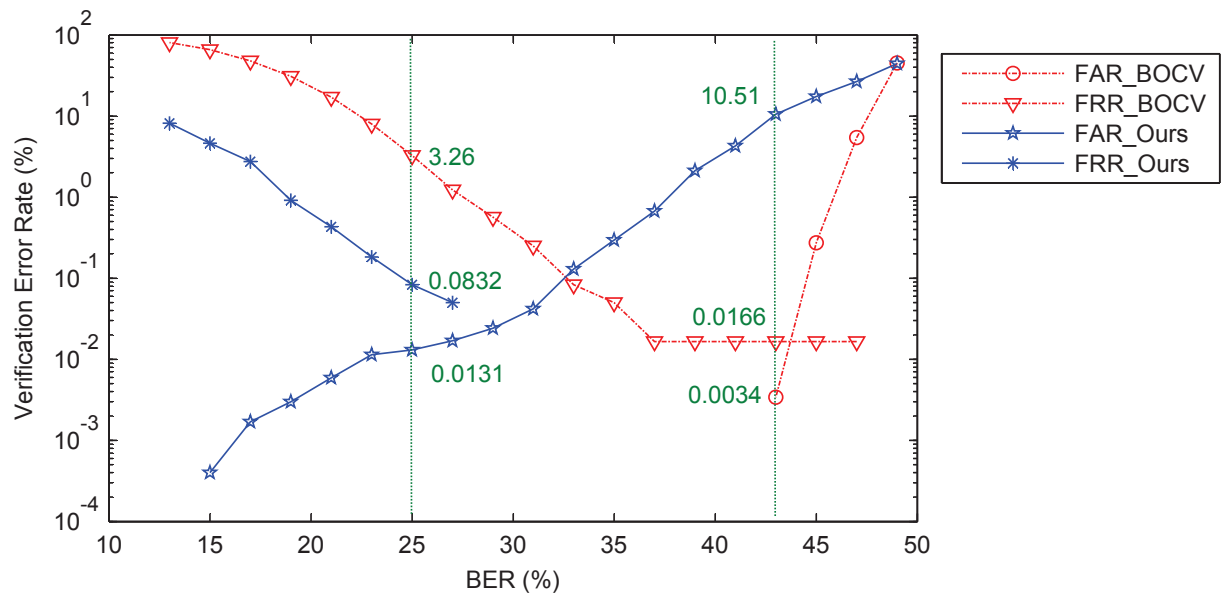

Fig. 6.10: Comparisons of verification error rate (\%) and their corresponding BER thresholds between the binary co-occurrence vector (BOCV) method and the proposed method (Ours).

separately on the verification accuracy. Compared with the popular coding based methods, the proposed approach achieves comparable verification error rate while much lower bit error rate of the genuine matching which gives a solution of combining palmprint verification and feature template protection scheme so that the system could be robust and accurate.

However, the extracted discriminative binary string is still not long enough to be secure. To alleviate the intra-class variations, how to extract the robust binary palmprint features of more bits so that a long key can be combined for security will be the point of our future work.

\subsection{Chapter conclusion}

In this chapter, a binary palmprint representation is presented. With regards to the research objective which we formulate in Section 1.3.3, this chapter aims to a fixed-length binary representation for palmprint template protection system. Firstly a real-valued feature vector is extracted based on the Gabor magnitude and phase responses. Then a one-bit quantization and bit selection approach is processed to achieve a binary string for representation. The FAR/FRR and the BER, which depends on an adjustable system threshold, are tested for determining the involved system parameters. Considering the 
error correcting ability of the current ECC technologies, the BER is expected to be lower than $25 \%$. Given a threshold so that the BER lower within the capability of ECC, the proposed method can achieve much lower FRR compared with the classical coding-based methods. In addition, the LDA is applied before bit extraction, which not only enhances the feature's discriminative ability but also leads to independent bits. 
Chapter

\section{Binary representation on multi-bit}

\section{quantization}

\subsection{Chapter introduction}

PURPOSE. This chapter introduces a binary representation for palmprint template protection system, which is based on the Gabor magnitude (GM) statistical features and multi-bit quantization. In Chapter 6, we present a binary palmprint representation which aims to be discriminative and stable. The false rejection rate and false acceptance rate (FRR/FAR), and the corresponding bit error rate (BER) are tested for the performance evaluation. In contrast, this chapter takes the secret key length into account, which depends on the BER performance and the error-correcting ability of used error correcting codes (ECC). Assuming that the Helper Data Scheme (HDS) with a BCH error correcting code is implemented, this chapter targets a binary representation which can achieve a low FRR/FAR and allows a long secret key to be combined. To reduce the likelihood of a key being obtained by an attacker's guesses, we expect that the allowed key length can be larger than 70 when the proposed binary palmprint representation is used for the considered HDS system. 


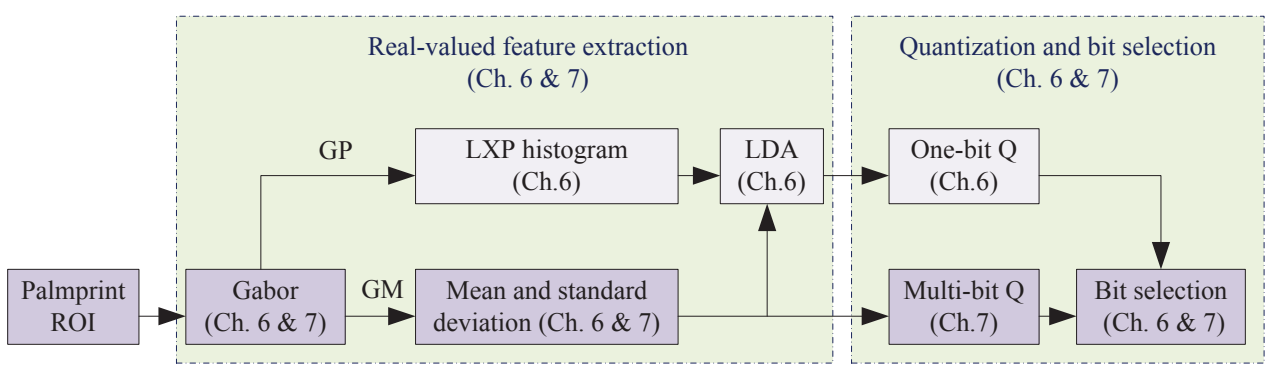

Fig. 7.1: Block diagram of our designed system, highlighting the context of Chapter 7 and its referred blocks.

CONTENTS. In this chapter, a method of extracting a fixed-length binary string is presented, which is based on the real-valued Gabor magnitude (GM) statistical features and a multi-bit quantization approach. In Section 7.2.3, a brief review on the GM statistical feature extraction is given. The generated real-valued feature vector is operated by a multi-bit equal-probability-interval quantization method. Some reliable bits are selected as the final palmprint representation based on a detection rate optimized bit allocation (DROBA) principle. The detailed quantization and bit selection approach is introduced in Section 7.2.4. In experiments, assuming that the BCH code is used for error correction so that the verification accuracy and the allowed maximum key length are tested. The comparison between the one-bit and multi-bit quantization method based on the same real-valued features is given. To obtain the independent bits, PCA/LDA is widely carried out before quantization. Thus, we conduct some contrast experiments to evaluate the effect of PCA/LDA process on the used real-valued features. Besides, the comparison is illustrated between the proposed method and the popular coding-based methods for unprotected palmprint verification system. In the context of system diagram, the content of this chapter and its referred blocks are highlighted in Figure 7.1.

PUBLICATION(s). The content of Section 7.2 of this chapter has been published in [105]. 


\subsection{Binary features on Gabor filtering and multi-bit}

\section{quantization}

\subsubsection{Abstract}

In this paper, we propose a method of fixed-length binary string extraction (denoted by LogGM_DROBA) from low-resolution palmprint image for developing palmprint template protection technology. In order to extract reliable (stable and discriminative) bits, multi-bit equal-probability-interval quantization and detection rate optimized bit allocation (DROBA) are operated on the real-valued features, which are resulted from representing the palmprint image by simple statistics on logarithmic transform of Gabor magnitude (LogGM). Assuming the Helper Data Scheme with a BCH error correction coding is adopted for template protection, the performance is evaluated on the Hong Kong PolyU palmprint database. The experimental results show that our method can achieve low Bit Error Rate (BER) resulted from genuine binary strings so that a long secret key (around 100 bits) is allowed to be combined for security, and low False Rejection Rate and low False Acceptance Rate (FRR/FAR) when the key retrieval process is considered as a Hamming distance classifier, which verify the high stability and strong distinctive ability of our extracted palmprint binary string.

\subsubsection{Introduction}

Biometric authentication technique, based on the natural linkage of biometric traits and individual identity, has received world-wide attention and gained significant development in the last decades. Varieties of biometric recognition systems are being used in the kinds of government and commercial applications around the world. Although most of them are successful, it raises concerns about system security and potential user privacy [106]. For higher levels of security, two main approaches have been developed to secure biometric template including biometric feature transformation by a one-way function, and binding the biometric template with a cryptographic key. As to the latter, the keyretrieval rate and allowed key length are the most important factors which can often be trade-off against each other to meet operational constrains. In this 
paper, our study is based on the Helper Data Scheme (HDS) for binding the palmprint template with a cryptographic key [107]. For its successful application of HDS, the biometrical traits are required to be presented as fixed-length binary strings, which are usually noisy due to the intra-class varieties. To bridge the gap between fuzziness of genuine biometric strings and exactitude of cryptographic key, Error Correction Coding (ECC) is designed to overcome the biometric variance. So far, most of template protection attempts, based on sorts of biometric modalities including iris, fingerprint, face, voice, and handwriting signatures, suffer from an excessive False Rejection Rate (FRR) - usually over $20 \%$, or a small allowed key length - smaller than 70 , which is unacceptable for practical applications [108]. The well-known difficulty is how to represent the biometrical traits as fixed-length binary strings, which can be of low Bit Error Rate (BER) for genuine samples, and of strong distinctive ability. The main obstacle is that most of genuine biometric strings provide BER as high as $40 \%$, which is far beyond the error-correcting capability of the currently existed ECC module (less than $25 \%$ ).

In this paper, a framework of binary feature extraction for palmprint template protection is surveyed, which includes real-valued feature extraction, binary quantization on single feature component and reliable bit selection. Emphasis is put on extracting reliable ( $i$. e. stable and discriminative) bits as a binary palmprint representation, which is expected to achieve low key-retrieval error rate (i. e. FRR and FAR), and provide low BER for genuine samples so that a secret key length of more than 70 is allowed to be bound, given the system works under the Helper Data Scheme with BCH codes for error correcting. The following issues are mainly considered:

(1) For the real-valued feature extraction from palmprint image, Gabor filtering is exploited in this study. For the typical palmprint recognition system (unprotected), the coding-based methods are considered to be most promising, which are generally based on the pixel-to-pixel quantization of wavelet filtered responses and can achieve high recognition accuracy [13]. However, for palmprint template protection system, those pixel-to-pixel encoded bits are too noisy to bind a secret key. Therefore, we resort to the sub-block partition based Gabor magnitude statistical features (denoted by LogGM) [55], which are expected to be more stable. In addition, for achieving bits of statistically independent and identically distributed bits so as to maximize the attacker's efforts in guessing the target template, we consider to perform PCA and LDA process respectively on LogGM before quantization [109]. Experimental results show that PCA/LDA transform causes that the discriminability of binary string improves while the bit stability heavily declines. 
(2) For fixed-length binary string generating, we take the one-bit and multibit quantization into consideration which are both based on the fixed equalprobability intervals so as to ensure the bits are identically distributed. After quantizing all the feature components into bits, we select the steady ones by some principle and then concatenate them into a string. Given the same binary string length, multi-bit quantizer could achieve more stable bits of lower BER than one-bit quantizer. While for better FRR/FAR performance, the selected bit number needs to be investigated. Here we resort to the detection rate optimized bit allocation principle (DROBA) for reliable bit selection [109]. The performance comparison with one-bit quantization will be presented in experiments.

(3) With regards to the performance evaluation, the secret key retrieval rate and the allowed maximum key size are considered as the most important factors in this study. Because the secure key retrieval process with an errorcorrecting operation can be modeled as a threshold classifier based on matching scores by the Hamming distance, we indicate the key retrieval performance by FRR and FAR, which is depended on the distinctive ability of the extracted bits. Given the enrolled and the genuine query palmprint binary strings are denoted by $B$ and $B^{\prime}$ respectively $\left(B\right.$ and $B^{\prime}=\{0,1\}^{n}$, and generally $B \neq$ $B^{\prime}$ due to the inevitable noisiness), the system will retrieve the secret key successfully in case $\left\|B-B^{\prime}\right\|<t$, under the assumption that the $\mathrm{BCH}(n, k, t)$ code is used for ECC, where $n$ is binary template size, $k$ is the allowed key length, and $t$ indicates the error correction capacity. Otherwise, a failure message is returned. The stability of binary strings is indicated by BER, which can be formulated as $\frac{\sum\left(B \oplus B^{\prime}\right)}{n} \times 100 \%$. In order to prevent the secret key from being guessed by exhaustive searching, $k$ is expected to be more than 70 .

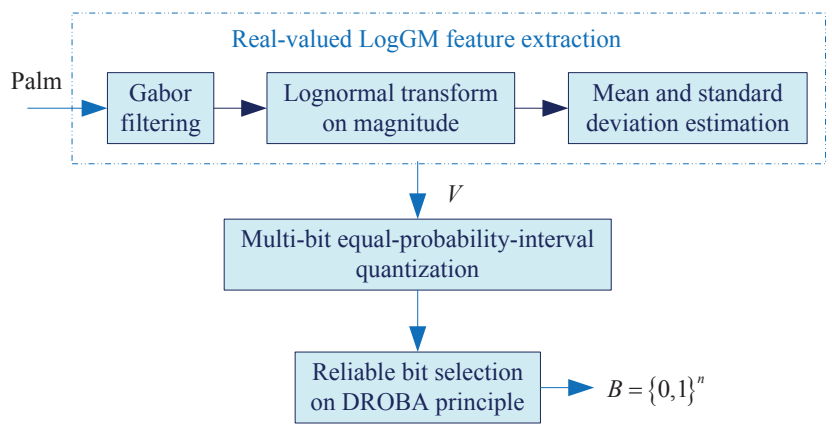

Fig. 7.2: The flow chart of our proposed binary feature extraction method for palmprint template protection. 
Taking all these factors into consideration, a novel binary palmprint feature extraction method based on Gabor statistical features and multi-bit fixedinterval quantization on DROBA (denoted by LogGM_DROBA) is proposed for template protection in this paper, whose flow chart is depicted in Fig. 7.2. Experimental results verify its high efficiency in terms of FAR, RRR, BER, and allowed secret key size given $\mathrm{BCH}$ codes are adopted, when compared with methods of the one-bit quantization on LogGM, LogGM and PCA/LDA based quantization, and the promising coding-based methods reported for unprotected palmprint recognition.

The reminder of this paper is organized as follows: in Sec. 7.2.3 the real-valued LogGM feature extraction algorithm is reviewed. Section 7.2.4 illustrates the proposed method (denoted by LogGM_DROBA) in detail. Section 7.2.5 presents experiments including the performance evaluation and contrast results on the HongKong PolyU palmprint database. Finally, conclusions and outlook are given in Sec. 7.2.6.

\subsubsection{Brief review of LogGM feature}

Gabor filter bank is a powerful tool for characterizing texture image features. Aiming to achieve the rotation and scale invariant image representation, the mean and standard deviation statistics resulted from Gabor responses are reported to be efficient. Inspired by those work, a method denoted by LogGM is presented for palmprint recognition based on the fact that the Gabor magnitude within each filtered subband uniformly approximates the Lognormal distribution [55]. The applied Gabor function is expressed as following:

$$
\begin{aligned}
g_{r, s}(x, y) & =\frac{1}{2 \pi \sigma^{2}} \exp \left\{\frac{-\left(x^{2}+y^{2}\right)}{2 \sigma^{2}}\right\} \\
& \times \exp \left\{2 \pi i\left(u_{r} x \cos \theta_{s}+u_{r} y \sin \theta_{s}\right)\right\} .
\end{aligned}
$$

$u_{r}$ is the frequency of sinusoidal wave along directional $\theta_{s}$ from $x$-axis, and $\sigma$ specifies the Gaussian envelope along $x$ and $y$ axes, which determines the bandwidth of the Gabor filter. Each Gabor function $g_{r, s}(x, y)$ with the parameters $\left(u_{r}, \theta_{s}, \sigma\right)$ is commonly transformed into a discrete Gabor filter and its direct current is turned to zero, which can be denoted by $\tilde{g}_{r, s}(x, y)$. Given an image $I(x, y)$, the Gabor magnitude (GM) can be expressed by $G M_{r, s}(x, y)=\|$ $\tilde{g}_{r, s}(x, y) * I(x, y) \|$. It has been empirically found that the lognormal densities 
fit the GMs very well, and the sub-blocks of each GM are also close to lognormal distribution. Accordingly, a group of Gaussian densities (LogGMs) can be obtained by $\log G M_{r, s}(x, y)=\log \left(G M_{r, s}(x, y)\right)$, the mean and standard deviation from which are exploited to construct the real-valued palmprint features $V=\left[v_{1}, v_{2}, \ldots, v_{m}\right]$. Following the experimental results in Ref. [55], five-scale and eight-frequency Gabor filters are carried out, and the mean and standard deviation are calculated from 21 sub-blocks. Therefore the number of feature components is $m=5 \times 8 \times 21 \times 2=1680$.

\subsubsection{Binary LogGM_DROBA feature extraction}

With regard to reliable bits extraction from LogGM feature $V=\left[v_{1}, v_{2}, \ldots, v_{m}\right]$, we resort to multi-bit quantization with equal-probability intervals and reliable bit selection on detection rate optimized bit allocation (DROBA) principle [109].

For a single feature component $v_{i}$, due to its inter- and intra-class variation, it can be modeled by a background probability density function (PDF) $p_{b}$, and a genuine user PDF $p_{g}$ to indicate the probability density of the whole population and the genuine user, respectively. Assuming $p_{b}$ and $p_{g}$ both approximate Gaussian distributions, we have $p_{b} \backsim G\left(v_{i}, \mu_{b}, \sigma_{b}\right)$ and $p_{g} \backsim G\left(v_{i}, \mu_{w}, \sigma_{w}\right)$ as the background PDF and the genuine user PDF respectively. As to the $b_{i}$-bit quantization for each $v_{i}, 2^{b_{i}}$ intervals are symmetrically constructed around the mean of the background PDF (usually $\mu_{b}$ and $\sigma_{b}$ are normalized into $\mu_{b}=0, \sigma_{b}=1$ ), with equally $2^{-b_{i}}$ background probability mass, which is independent of the genuine user PDF. Gray codes are allocated for each interval so that the Hamming distance between two adjacent quantization intervals is limited to one which results in a better performance of a Hamming distance classifier. The feature component $v_{i}$ derived from genuine user is expected to fall into one interval which is referred to as the genuine interval. Figure 7.3 gives an illustration of $b_{i}$-bit equal-probability-interval quantization as we described when $b_{i}=2$.

Empirically we know that the stability and discriminability differ for different feature components, i. e. $p_{g}$ differs in $\left(\mu_{w}, \sigma_{w}\right)$ as $v_{i}$ varies. Therefore, we choose to allocate more bits for the more stable and discriminative components and less for the others, in case the binary string length is fixed. The quantization performance of $v_{i}$ with $b_{i}$-bit quantization can be defined as the theoretical FAR $\alpha_{i}$, FRR $\beta_{i}$ and the corresponding detection rate $\delta_{i}$ by the 


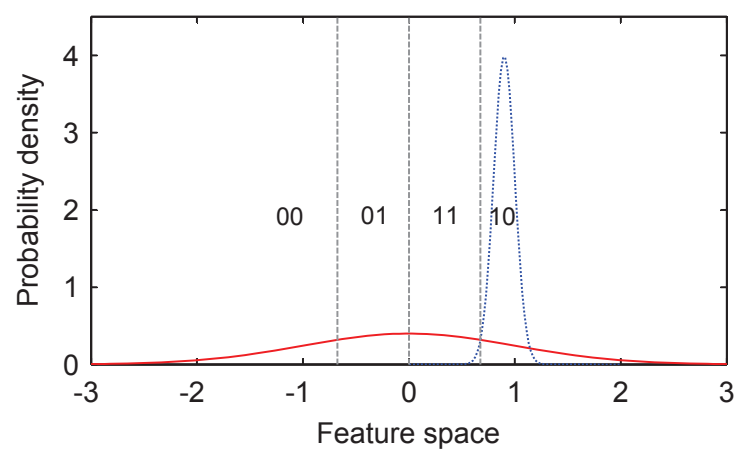

Fig. 7.3: Illustration of the two-bit equal-probability-interval quantization for one feature component $v_{i}$. Gray codes are used for coding each interval. The background PDF $p_{b}\left(v_{i}, 0,1\right)$ (solid line); the genuine user PDF $p_{g}\left(v_{i}, \mu_{w}, \sigma_{w}\right)$ (dot line, $\mu_{w}=0.9, \sigma_{w}=0.1$ is taken as an example here); the quantization intervals (dash line).

following expressions:

$$
\begin{gathered}
\alpha_{i}\left(b_{i}\right)=\int_{Q_{\text {genuine }, i}\left(b_{i}\right)} p_{b}\left(v_{i}\right) d_{v_{i}} \\
\delta_{i}\left(b_{i}\right)=\int_{Q_{\text {genuine }, i}\left(b_{i}\right)} p_{g}\left(v_{i}\right) d_{v_{i}} \\
\beta_{i}\left(b_{i}\right)=1-\delta_{i}\left(b_{i}\right)
\end{gathered}
$$

where $Q_{\text {genuine, } i}\left(b_{i}\right)$ represents the genuine user interval when $b_{i}$-bit quantization is carried out.

In this paper, about the LogGM feature $V$, we have $m$ feature components. For $b_{i}$-bit quantization, $b_{i} \in\{0,1,2,3\}$ is considered. Let $n$ denote the number of selected reliable bits, $n \in\{127,255,511,1023\}$ is investigated complying with the string form of $\mathrm{BCH}$ error correcting code, and $70<n<1680$ is expected. In this paper, $2^{69}$ security is sought, e. $g$. the secret key $k$ size is expected to be larger than 70 . That is why $n>70$ is expected. According to the DROBA principle, the optimal bit assignment $\left\{b_{i}^{*}\right\}$, which indicates the number of quantization bits for every single feature $\left\{v_{i}\right\}, i=1, \ldots, m$, can be expressed as:

$$
\left\{b_{i}^{*}\right\}=\arg \max _{\sum_{i=1}^{m} b_{i}=1} \delta\left(b_{1}, \ldots, b_{m}\right)
$$


Assuming that all the $m$ feature components are independent, Eq. (7.5) can be rewritten as:

$$
\left\{b_{i}^{*}\right\}=\arg \max _{\sum_{i=1}^{m} b_{i}=1} \prod_{i=1}^{m} \delta_{i}\left(b_{i}\right)
$$

To solve this optimization problem, we applied the Greedy Search approach presented in Ref. [109]. With regards to the calculation of detection rate $\delta_{i}\left(b_{i}\right)$, we firstly subtract the mean and normalize the standard deviation on the entire enrolled samples so that $p_{b, i}$ approximate $G\left(v_{i}, 0,1\right)$ density. Then the mean $\mu_{w}$ and the standard deviation $\sigma_{w}$ are estimated from the enrolled samples for each palm respectively.

Finally, following the knowledge of $\left\{b_{i}^{*}\right\}$, for each feature component, we carry out the $b_{i}$-bit $\left(b_{i} \in\{0,1,2,3\}\right)$ equal-probability-interval quantization by coding interval with the Gray code, and then concatenate them to generate a binary string $B=\{0,1\}^{n}$ of length $n$ as our proposed binary palmprint representation.

\subsubsection{Experimental results}

\subsubsection{Experimental setup}

The HongKong Polytechnic University (PolyU) palmprint database is used to test our proposed method [6]. They were captured by a CCD camera from 386 different palms and collected in two sessions with two different illumination conditions. There are 3889 images in session one and 3863 samples in session two respectively. Because there is one palm which has only one sample captured in session two, we use the other 385 palms for our experiments. The resolution of original captured images is $384 \times 284$ pixels at 75 dpi. By preprocessing each captured image (as shown in Fig. 7.4), the central region size of $128 \times 128$ is used for feature extraction.

For the training-evaluation-set split in all the experiments, 185 palms are randomly chosen for training and the remaining 200 palms for evaluation, which is repeated six times. For training, all the samples (around ten) from the same palm in session one are used. For evaluation, all the samples are from session two. About the enrollment-test split on the samples from one palm, five samples are randomly selected for enrollment and the remaining ones for 

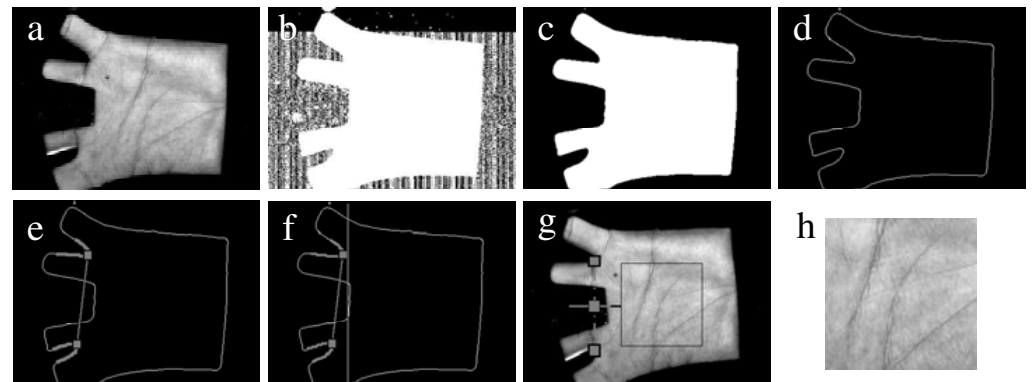

Fig. 7.4: (a) A typical palmprint image from HongKong PolyU Palmprint Database; (b)-(h) illustrate the registration and region-crop processes we carried out.

Table 7.1: Examples of the BCH codes and their corresponding error correcting capability. Codeword size $(n)$, secret key length $(k)$, and the correctable bits $(t)$.

\begin{tabular}{|l|c|}
\hline BCH $(n, k, t)[$ bits] & Error correcting capability $\left(\frac{t}{n}\right)$ \\
\hline \hline$(127,71,9)$ & $7.09 \%$ \\
$(255,71,29)$ & $11.37 \%$ \\
$(511,76,85)$ & $16.63 \%$ \\
$(1023,76,187)$ & $18.28 \%$ \\
\hline
\end{tabular}

test. All the test samples in the test set are matched with the enrolled samples by Hamming distance so that totally we get 6043 genuine scores and 1,202,557 imposter scores.

\subsubsection{Performance evaluation}

To evaluate the key-retrieval capability of our proposed binary palmprint representation (denoted by LogGM_DROBA) when utilized for template protection system, we assume the Helper Data Scheme with the BCH code for error correcting is carried out. Table 7.1 shows some examples of $(n, k, t)$ settings for $\mathrm{BCH}$ code. $n$ is an integer of the form $2^{N}-1$ for some integer $N>2$. To ensure higher security of the key, the $\mathrm{BCH}$ codes with $k>70$ are considered in our experiments. Note, $t$ increases as $n$ grows but lies lower than $20 \%$ of the binary vector. The stability and discriminability of the LogGM_DROBA string are dependent on the number of selected reliable bits. To investigate the impact of the binary string length on the FAR, FRR, and $k$ size, we evaluated the verification performances at various binary string lengths.

The ROC curves are shown in Fig. 7.5 for the proposed binary representation method, given $n$ is set to $127,255,511$ and 1023 respectively. As can be 


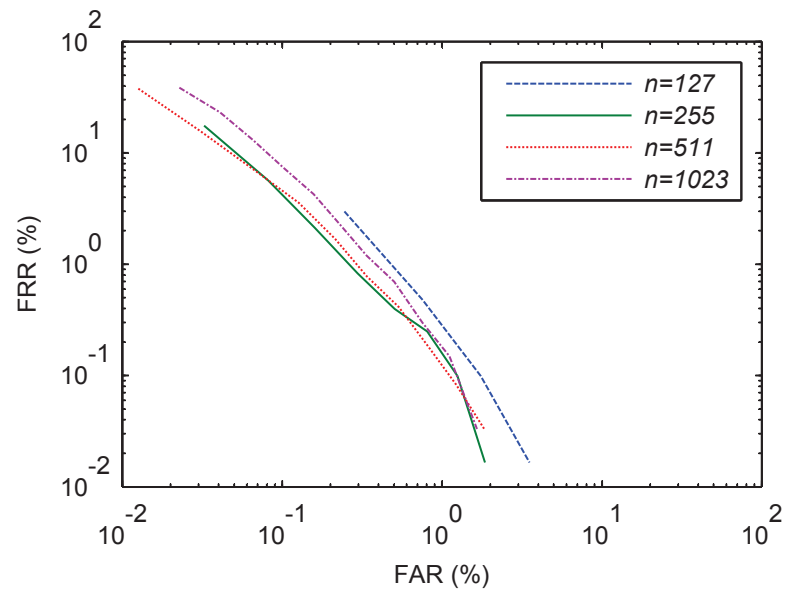

Fig. 7.5: The ROC performances of the proposed binary string extraction method when the string length $n$ is set to $127,255,511$ and 1023 respectively.
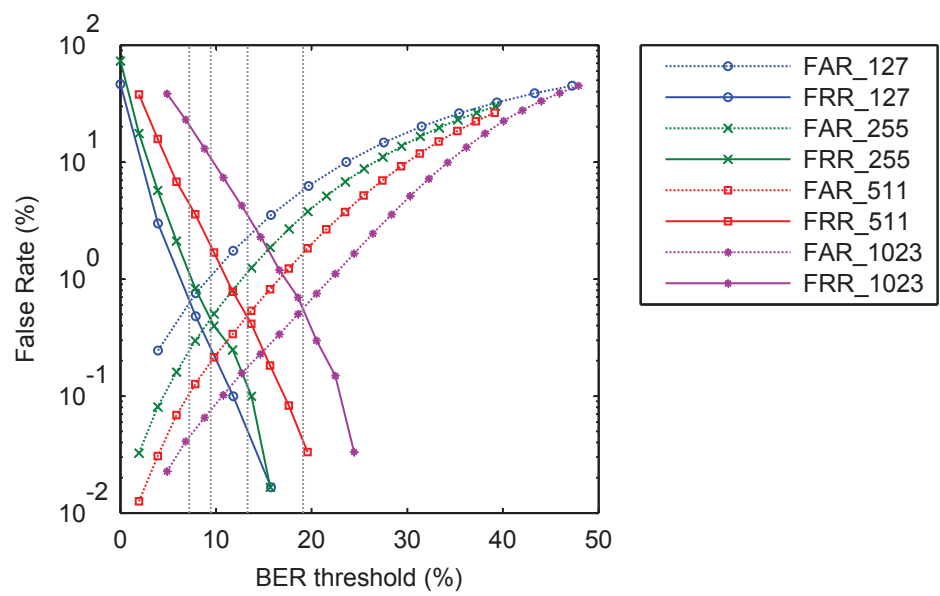

Fig. 7.6: The FAR and FRR performances of the proposed binary string extraction method when the string length $n$ is set to $127,255,511$ and 1023 respectively. 
seen from it, the performance of bit discriminative ability firstly improves and then starts to degrade as $n$ increases from 127 to 1023 . The improvement could be because more discriminant information is exploited when more realvalued LogGM feature components are selected for extracting bits. However, as the number of selected feature components for bit extraction increases, the discriminability of binary string decreases. It could be explained that the computed detection rate following Eq. (7.3) is less accurate when the selected feature component for bit extraction is less reliable, $i$. e. its statistical density is more far away from the Gaussian model assumption. The results shown in Fig. 7.5 suggest that the moderate string length $n$ can be 255 or 511 . Besides the discriminability, the stability of bit string is the other important factor, which can be indicated by BER of the genuine matching. Given the string length $n$, the smaller the BER is, $i$. e. the less error bits, the larger the allowed key size is. Figure 7.6 plots the FAR and FRR performances versus the BER threshold which is depended on the ECC module and bounded by the applied BCH codes. As can be seen from it, the BER threshold increases as $n$ grows from 127 to 1023 at their corresponding equal error rate (EER) points, where FAR is equal to FRR. However, the error correcting capability also increases as $n$ grows. Corresponding to the results shown in Fig. 7.6, we tabulate the performance comparison in Table 7.2. As it shows, the proposed method achieves the best performance at $n=255$, where the EER is $0.45 \%$ corresponding to $9.41 \%$ of the BER threshold. By consulting the BCH code dictionary, it is figured out that the allowed maximum key size is $k=99$. In reality, FAR is required to be much lower for security. Here, the performance at the point of $\mathrm{FAR}=0.1 \%$ is also listed in Table 7.2. As it shows, our method can achieve the lowest $\mathrm{FRR}(3.97 \%)$ at $n=255$, where the maximum $k=163$ according to the $\mathrm{BCH}$ codes.

\subsubsection{Compared with other methods}

In this section, the results of contrast experiments are presented.

(1) First, we consider the one-bit quantization on the LogGM feature components, for which the reliable bit selection is based on the mean $\mu_{w}$ of genuine PDF after the background PDF is normalized to zero mean and unit standard deviation $\left(\mu_{b}=0, \sigma_{b}=1\right)$. Figure 7.7 shows the comparison of performances between the one-bit quantization and our proposed DROBA based multi-bit quantization when the binary string length $n$ is set to 63,255 , and 1023 respectively. As can be seen from Fig. 7.7 (a)-(c), given $n=255$, DROBA based 
Table 7.2: Performance of the proposed method when the string length $n$ varies. Key size $(k)$. Correctable bits $(n)$.

\begin{tabular}{|l|c|c|c|c|}
\hline$n$ & 127 & 255 & 511 & 1023 \\
\hline \hline EER (\%) & 0.62 & 0.45 & 0.49 & 0.58 \\
\hline $\begin{array}{l}\text { BER threshold } \\
\frac{t}{n} \text { @ EER point }\end{array}$ & $7.09 \%$ & $9.41 \%$ & $13.31 \%$ & $19.26 \%$ \\
\hline $\begin{array}{l}\text { Allowed maximum } \\
k \text { @ EER point }\end{array}$ & 71 & 99 & 76 & 46 \\
\hline $\begin{array}{l}\text { FRR (\%) } \\
\text { @ FAR=0.1\% }\end{array}$ & 12.89 & 3.97 & 4.68 & 7.81 \\
\hline $\begin{array}{l}\text { BER threshold } \\
\frac{t}{n} \text { @ FAR=0.1\% }\end{array}$ & $1.57 \%$ & $4.71 \%$ & $7.05 \%$ & $10.56 \%$ \\
\hline $\begin{array}{l}\text { Allowed maximum } \\
k \text { @ FAR=0.1\% }\end{array}$ & 106 & 163 & 241 & 228 \\
\hline
\end{tabular}

multi-bit quantization outperforms one-bit quantization. But when $n=63$ and 1023, one-bit quantization achieves better verification performance. From Fig. 7.7 (d)-(f) we can know that DROBA based multi-bit quantization can achieve better BER performance than one-bit quantization at the same string length $n$. Accordingly, we can conclude that the proposed method can achieve more steady binary strings than LogGM based one-bit quantization method, given the same $n$. However, with regards to the discriminability of string, our method performs better only when $n$ is a moderate value.

(2) For our proposed method, the stability and discriminability of binary string are given the top priority. However, dependency of bits is another main concern for security. To obtain independent bits, PCA/LDA projection is widely operated on the real-valued features before quantization [109]. Here, for comparison we carry out PCA and LDA respectively on the LogGM features and then process the one-bit quantization. Due to the LDA process, the number of obtained real-valued feature components is 184 (185 palms for training). Therefore, $n(70<n<184)$ is set to 127 for all the compared methods in this experiment. As Fig. 7.8 shows, PCA/LDA process leads to better FAR and FRR performance but worse BER. According to Table 7.2, we know that BER threshold should be lower than about $7 \%$ so that a key could be combined into the binary string. But the PCA/LDA results in a BER of more than $30 \%$ at the EER point, which is far beyond the error correcting capability. When the $\mathrm{BCH}$ code is operated on the binary string resulted from the features with a PCA/LDA process, the system will derive a large FRR, which is beyond the level acceptable for practical use.

(3) For the unprotected palmprint verification system, many coding based 


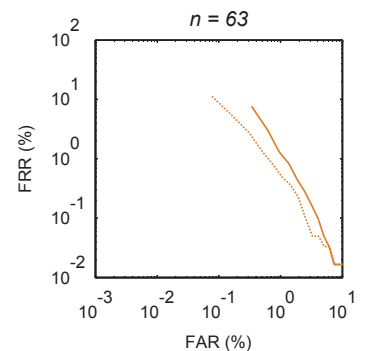

(a)

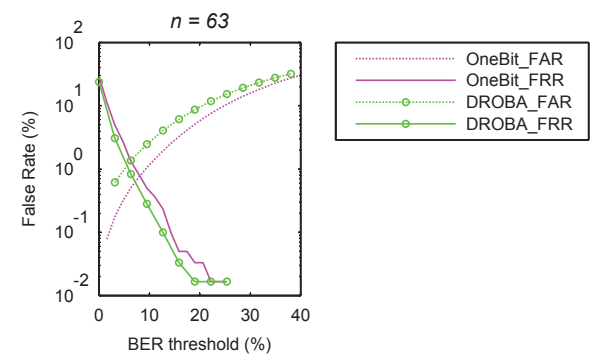

(d)

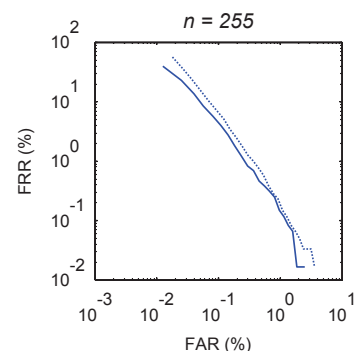

(b)

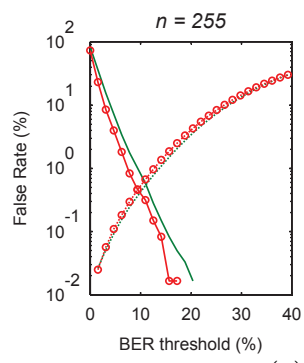

(e)
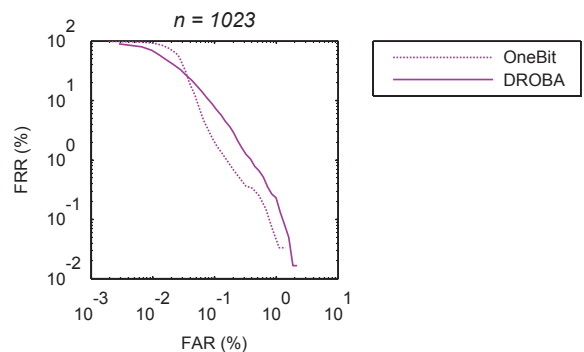

(c)

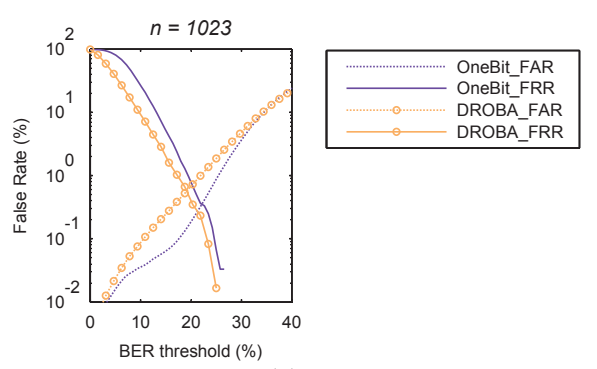

(f)

Fig. 7.7: Performance comparison between one-bit quantization and DROBA based multi-bit quantization (our proposed method) on the same $\operatorname{LogGM}$ features. The binary string length $n$ is set to 63,255 and 1023 respectively. 

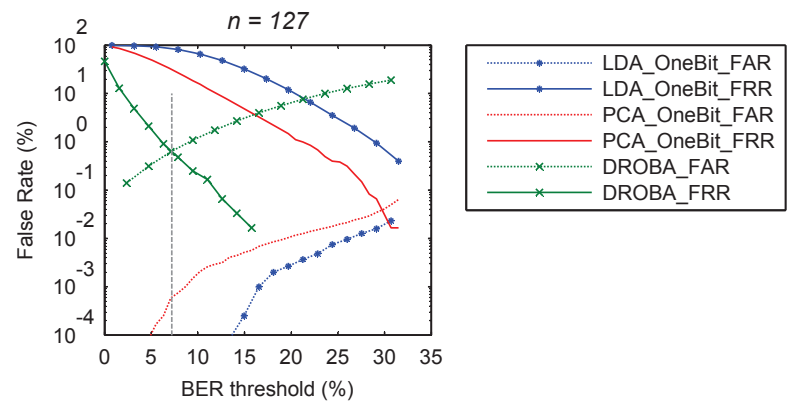

Fig. 7.8: Performance comparison of FAR/FRR and BER. Here, the binary string length $n$ is set to 127 for all of the compared methods.
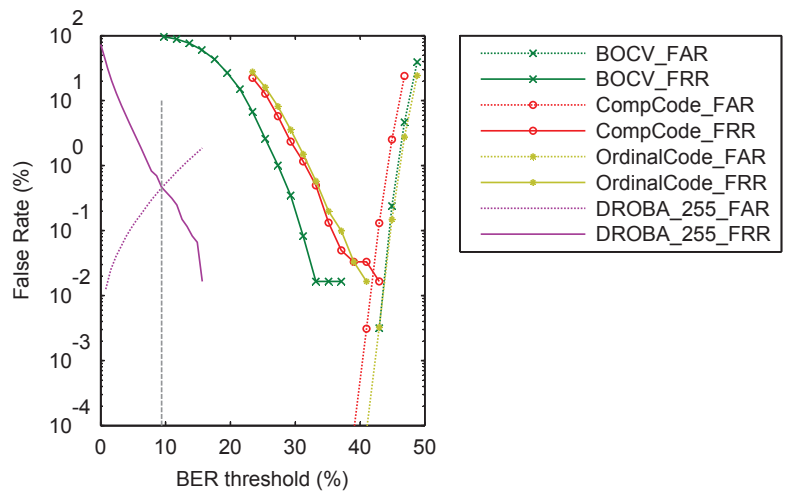

Fig. 7.9: Performance comparison of FAR/FRR and BER between the proposed method and there coding based methods. Here, the binary string length $n$ is set to 255 for the proposed method (denoted by DROBA_255). For others, $n=32 \times 32=1024$. 
methods have been reported with great FAR/FRR performance such as CompCode [37], Ordinal code [94], BOCV [41] and so on, which represent palmprint by several binary matrices. In order to get the matching score, it is required to shift the whole code matrix by several pixel horizontally and vertically, and match multiple times. In this experiment, each bit matrix is down-sampled by ratio of $4: 1$ into a plane size of $32 \times 32$. The shift range is set to $[-2,2]$. Figure 7.9 shows the comparison of FRR/FAR and BER performance between them and our proposed method. As can be seen from it, in contrast to our proposed method, the coding based methods can achieve lower FRR/FAR but much higher BER (up to 40\%). Therefore, although the binary features from coding based methods are highly discriminative, they are not steady enough to be used for template protection system. In addition, the coding based methods require code matrix to shift multiple times for matching score calculation, which challenges the combination of palmprint verification and the template protection.

\subsubsection{Conclusion and outlook}

In this paper a novel binary palmprint feature (bits) extraction method for improving template protection technology is presented. In order to achieve a binary string of high stability and strong distinctive ability, real-valued features are firstly extracted based on the statistical characters of Gabor magnitude and then multi-bit quantization on detection rate optimized bit allocation (DROBA) principle is carried out. Experimental results demonstrate that the proposed method can achieve high key-retrieval accuracy $(i$. e. low FRR/FAR) and a long secret key (more than 70 bits) is allowed to be bound for higher security due to its low BER of genuine strings, given a moderate binary string length.

From the contrast experiments, we can conclude that multi-bit quantization on DROBA uniformly achieves lower BER than one-bit quantization in case that both of them are based on LogGM features and the selected reliable bit number is set to the same. However, for the better FRR/FAR performance, the string length $n$ needs to be moderate. A small $n$ leads to weak discriminability because many real feature components are not used for bits generation so that some discriminative information is lost. However, a large $n$ also results in poor FRR/FAR performance because some real feature components of less reliable are exploited, whose statistical density might be far away from the Gaussian model assumption so that the computation of detection rate is less 
correct. LDA/PCA process can provide bits of more independent and better verification accuracy, but leads to worse BER so that a secret key of long length can not be bound. In addition, the well-known coding based methods for unprotected palmprint recognition system have great power to distinguish individuals. But it is challenging to apply them for template protection system because the binary strings from genuine samples they generate provide a high BER which is far beyond the error correcting capability of currently existing ECC technology.

In this paper, emphasis is put on the stability and distinctive ability of extracted bits for template protection system. However, for higher security, the bits independence or correlation issue, the bits error pattern and their corresponding suitable error-correcting codes could be concerned in our future work.

\subsection{Chapter conclusion}

With regards to the research objective which we formulate in Section 1.3.3, this chapter aims to a fixed-length binary representation for palmprint template protection system. Compared with Chapter 6, this chapter supplements the allowed maximum key length $k$ as the performance indicator besides the verification FRR/FAR and their corresponding BER. The key length $k$ depends on the BER and the exploited ECC error-correcting ability. In this chapter, we assume that the $\mathrm{BCH}$ code is used for error correcting. By choosing different $\mathrm{BCH}$ codes, which determines the selected bits number for palmprint representation and the error-correcting capability, the achieved FRR/FAR and the allowed maximum key length $k$ vary. Experiments show that the proposed method can achieve low FAR/FRR and allows long secret key to be combined. Chapters 6 and 7 both investigate the Gabor magnitude (GM) information for the real-valued feature extraction. The LDA is adopted in Chapter 6. But the experiments in this chapter show that the LDA process can provide bits of more independent and better verification accuracy, but it leads to worse BER so that a secret key of long enough can not be bound. Thereafter, the proposed method does not include LDA process. Consequently, the bits independence issue remains to be concerned in the future work, as well as the bits error pattern and their corresponding suitable error-correcting codes. 
$\Gamma_{\text {Chapter }}$

\section{Conclusions and Future Work}

In order to conclude this thesis, we will first address the research objectives formulated in Chapter 1, and then summarize the contributions of this thesis. Some discussions on the solutions we present in this thesis will be given regarding the defined research objectives. Furthermore, we will present the further work direction.

\subsection{Objectives and contributions}

The context of this research is the development of online palmprint recognition applications. As Section 1.3 presents, the basic research question is:

How should we create the palmprint representations and their corresponding similarity measurements, which can distinguish the interclass palmprints and meanwhile can be robust against the intra-class variations of translation, rotation, and illumination perturbation?

The target application scenarios are:

I. Typical (unprotected) online palmprint recognition system; 
Table 8.1: The refined questions and corresponding research targets, given low-resolution palmprint images.

\begin{tabular}{|l|l|}
\hline Refined questions & Research targets \\
\hline \hline \multirow{2}{*}{$\begin{array}{l}\text { Part A: How should we } \\
\text { construct the feature } \\
\text { representation for a } \\
\text { recognition system? }\end{array}$} & i. Robust to translation and rotation \\
\cline { 2 - 2 } & ii. Robust to illumination variation \\
\cline { 2 - 2 } & iii. Discriminative (High recognition rate) \\
\cline { 2 - 2 } $\begin{array}{l}\text { Part B: How should we } \\
\text { construct the feature } \\
\text { representation for a } \\
\text { template protection } \\
\text { system? }\end{array}$ & i. Small feature size \\
\cline { 2 - 2 } & i. Fixed-length bit string \\
\cline { 2 - 2 } & iii. Low bit error rate of genuine binary templates \\
\cline { 2 - 2 } & FRR/FAR) \\
\hline
\end{tabular}

II. Combine the palmprint recognition system with template protection schemes;

By analyzing the conditions of these two applications, the research objectives are summarized as two parts:

A. Real-valued invariant feature representation for the unprotected palmprint recognition system;

B. Binary reliable feature representation for the palmprint template protection system.

In accordance to these two objectives, the basic research question is refined by some specific system requirements. Table 8.1 lists the refined questions and corresponding system requirements. To explore the solutions, the texture analysis techniques are investigated in this thesis. Figure 8.1 displays our designed strategies, which lead to the main contributions of this thesis. The solutions and achievements to the refined questions are listed in Tables 8.2 and 8.3 corresponding to Parts $\mathrm{A}$ and $\mathrm{B}$ respectively.

\subsection{Discussion of achievements}

To summarize, in this thesis, we present solutions to extract features from low-resolution palmprint images. The texture analysis technologies are mainly investigated.

With regards to the real-valued invariant feature extraction, the designed 


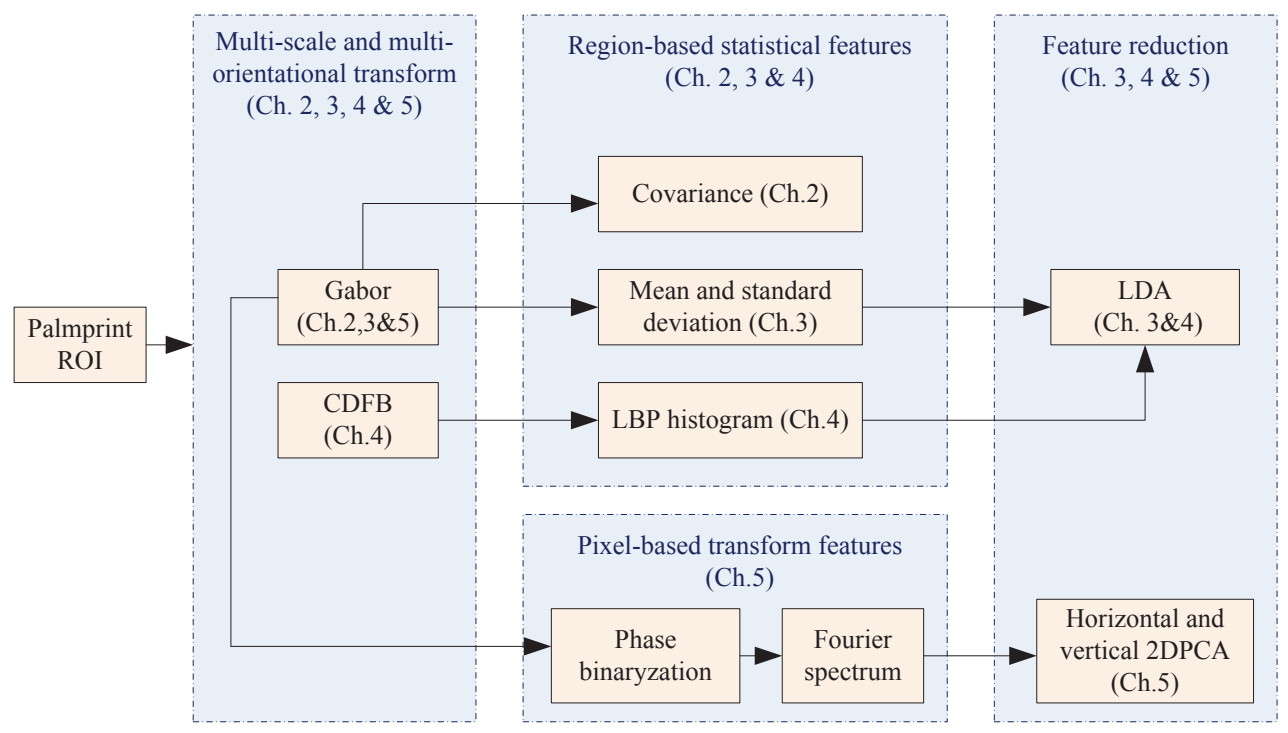

(a) Part A: Real-valued invariant feature extraction for the unprotected palmprint recognition system.

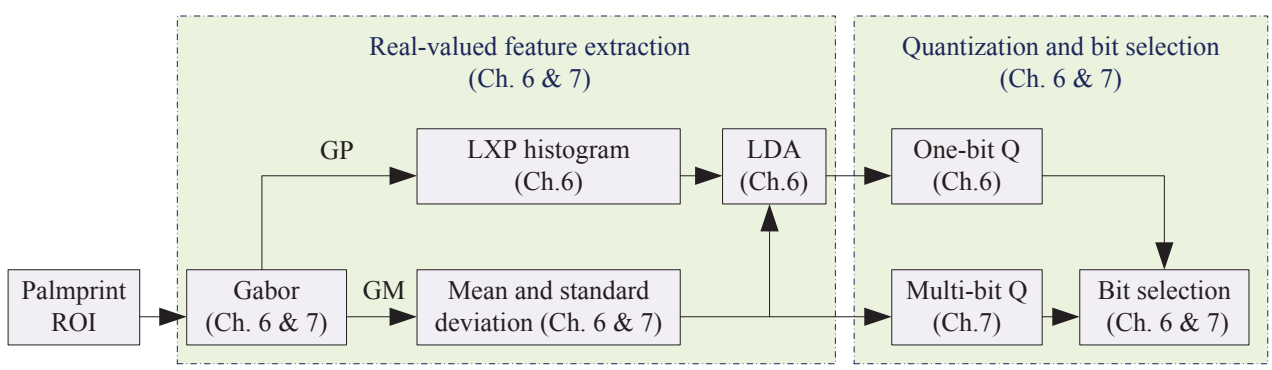

(b) Part B: Binary reliable feature extraction for the palmprint template protection system.

Fig. 8.1: Block diagram of our designed system for the research objectives Part B and A respectively, focusing on the main contributions of this thesis. (ROI - region of interest; LBP - local binary pattern; LDA - linear discriminant analysis; 2DPCA - two-dimensional principle component analysis; LXP - local xor pattern; Q - quantization) 
Table 8.2: Solutions and corresponding achievements to the refined research question A: How should we construct the feature representation for a recognition system.

\begin{tabular}{|l|l|}
\hline Solution & Achievements \\
\hline \hline Ch.2: Region Covariance Matrices (RCM) & i. Robust to translation and rotation \\
\cline { 2 - 2 } $\begin{array}{l}\text { as representation based on Log-scaled } \\
\text { Gabor magnitude and Gabor phase }\end{array}$ & ii. Robust to illumination variation \\
\cline { 2 - 2 } responses & iii. Discriminative (High recognition rate) \\
\cline { 2 - 2 } $\begin{array}{l}\text { Ch.3: Mean and standard deviation as } \\
\text { representation based on Log-scaled Gabor }\end{array}$ & i. Small feature size \\
\cline { 2 - 2 } magnitude responses & iii. Dobust to translation and rotation \\
\cline { 2 - 2 } & iv. High feature matching speed \\
\cline { 2 - 2 } $\begin{array}{l}\text { Ch.4: Local pattern histogram statistical } \\
\text { representation based on the complex }\end{array}$ & v. Small feature size \\
\cline { 2 - 2 } directional filter bank (CDFB) transform & iii. Discriminative (High recognition rate) \\
\cline { 2 - 2 } and local binary pattern (LBP) operation & iv. High feature and matching speed \\
\cline { 2 - 2 } & v. Small feature size \\
\hline \multirow{4}{*}{$\begin{array}{l}\text { Ch.5: Fourier spectral representation } \\
\text { based on Gabor phase coding }\end{array}$} & i. Robust to translation and rotation \\
\cline { 2 - 2 } & ii. Robust to illumination variation \\
\cline { 2 - 2 } & iii. Discriminative (High recognition rate) \\
\cline { 2 - 2 } & iv. High feature and matching speed \\
\cline { 2 - 2 } & v. Small feature size \\
\hline
\end{tabular}

Table 8.3: Solutions and corresponding achievements to the refined research question B: How should we construct the feature representation for a template protection system.

\begin{tabular}{|l|l|}
\hline Solution & Achievements \\
\hline \hline \multirow{2}{*}{$\begin{array}{l}\text { Ch.6: Binary representation by one-bit } \\
\text { quantization on the features from }\end{array}$} & i. Fixed-length bit string \\
\cline { 2 - 2 } $\begin{array}{l}\text { Log-scaled Gabor magnitude and Gabor } \\
\text { phase responses }\end{array}$ & $\begin{array}{l}\text { ii. Low BER of genuine binary templates } \\
\text { rate: FRR/FAR) }\end{array}$ \\
\cline { 2 - 2 } & $\begin{array}{l}\text { iv. Alignment operation between tem- } \\
\text { plates is limited }\end{array}$ \\
\hline $\begin{array}{l}\text { Ch.7: Binary representation by multi-bit } \\
\text { equal-probability-interval quantization on } \\
\text { the features from Log-scaled Gabor } \\
\text { magnitude responses }\end{array}$ & i. Fixed-length bit string \\
\cline { 2 - 2 } & $\begin{array}{l}\text { ii. Low BER of genuine binary templates } \\
\text { iii. Discriminative (Low verification error } \\
\text { rate: FRR/FAR) }\end{array}$ \\
\cline { 2 - 2 } & $\begin{array}{l}\text { iv. Alignment operation between tem- } \\
\text { plates is limited }\end{array}$ \\
\hline
\end{tabular}


strategy consists of three procedures: i. Multi-scale and multi-orientational transform; ii. Region-based statistical or pixel-based feature extraction; iii. Feature reduction. In accordance to this strategy, four methods have been proposed: (1) RCM representation; (2) Mean and standard deviation representation; (3) LBP histogram representation; (4) PalmCode's Fourier spectrum representation. Methods (1) and (2) both involve a large group of Gabor filtering during the feature extraction. Thus, the feature extraction speed is low and the storage requirement is high. Further, the similarity calculation of RCM is time consuming so that the feature matching speed is low. In addition, the RCM representation is a training-free method and it is relatively robust to illumination variations. While the mean and standard deviation representation method involves a training procedure, and it turns out to be sensitive to illumination variations. Method (3) is proposed by addressing the drawbacks of the large group of Gabor filtering and other multi-scale and multi-orientational transforms. It explores the CDFB transform and the LBP histograms are extracted as features. This method achieves the comparable recognition rate but outperforms significantly in terms of execution speed and feature size. Different from methods (1), (2) and (3), which explore the region-based statistical features for being robust to image translation and rotation, method (4) investigates the pixel-based transform features. A Fourier spectral representation is proposed based on the classical method PalmCode. PalmCode is reported to provide good discriminative ability and robustness to illumination changes, but it is sensitive to the image translation. Therefore, we resort to the Fourier transform and the horizontal and vertical 2DPCA transform to process the PalmCode further, due to their translation invariant property. Consequently, the method (4) achieves the best recognition performance considering the recognition rate, computational speed and feature size.

With regards to the binary reliable feature extraction for template protection system under the Helper Data Scheme (HDS), we consider to extract the region-based statistical features and then quantize them into bits. Accordingly, two methods are presented in this thesis. Both of them put emphasis on the stability and distinctive ability of extracted bits. Method (1) achieves low verification error rate (FRR/FAR) and low bit error rate (BER) so that the key-retrieval error rate is low. Method (2) is proposed by taking the secret key length into account, which partly influences the system's security. By assuming the applied error correcting code (ECC) is BCH code, method (2) achieves high key-retrieval accuracy $(i$. e. low FRR/FAR) and a long secret key (more than 70 bits) is allowed to be bound for higher security. 


\subsection{Future work}

Further research work on online palmprint recognition can focus on the following aspects:

- Improving image quality: In this work, we investigate how to represent the low-resolution images so that the within-class variations can be minimized and the inter-class differences can be maximized. The explored strategy is the texture analysis technique. The considered within-class variances mainly include image translation, rotation and illumination changes. However, the targeted image variances might be suppressed by improving the quality of input images. For instance, we can improve the palmprint image capture environment, or improve the ROI extraction algorithm. Besides, due to the advanced sensor technology, there are other kinds of palmprint images reported, such as multispectral palmprint and 3D palmprint. They can provide more rich image information so that the recognition performance could be enhanced.

- Fusing multiple biometric modalities: Palmprint, as a kind of handbased character, is promising to fuse other biometrical modalities for higher recognition accuracy, such as fingerprint and finger vein pattern. In addition, the solutions present in this thesis can also be explored for other texture-based image representation, such as iris, face and finger vein pattern.

- Improving template protection technology: In the research field of biometric template protection, the well-known difficulty is that there is a big gap between fuzziness of genuine biometric strings and exactitude of cryptographic key. In the Helper Data Scheme (HDS), error correcting code (ECC) is designed to overcome the biometric data variance. However, most of the current template protection attempts, based on sorts of biometric modalities including iris, fingerprint, face, voice and handwriting signatures, suffer from an excessive false rejection rate or a small allowed key length, which is unacceptable for practical applications. In this thesis, the solutions to provide a reliable biometric string from palmprint for HDS system is given. For better performance, more reliable bits extraction methods are to be investigated on one hand, involving more efficient real-valued feature extraction approach and more suitable bit quantization and selection strategy. On the other hand, more advanced ECC technology is desirable to compensate for the fussiness of biometric data, which involves the investigation of bit independence or correlation issue, the bits error pattern and so on. 


\section{References}

[1] "ISO/IEC JTC1 SC27. FCD 24745 - information technology - security techniques - biometric template protection," 2010.

[2] P.T. Tuyls, B. Skoric, and T.A.M. Kevenaar (Eds.), Security with Noisy Data - On Private Biometrics, Secure Key Storage and AntiCounterfeiting. Springer, 2007.

[3] A. Jain, K. Nandakumar, and A. Nagar, "Biometric Template Security," EURASIP Journal on Advances in Signal Processing, vol. 2008, pp. 1$18,2008$.

[4] A. Jain, P. Flynn, and A. Ross, Handbook of Biometrics. Springer, 2007.

[5] A. Jain and J. Feng, "Latent Palmprint Matching," IEEE Trans. Pattern. Anal. Mach. Intell., vol. 31, no. 7, pp. 1032-1047, 2009.

[6] "Biometrics research center (BRC) in HongKong." [Online]. Available: http://www.comp.polyu.edu.hk/ biometrics/

[7] F. Galton, Fingerprints. Wm. S. Hein Publishing, 2002.

[8] D. Zhang, W. Kong, J. You, and M. Wong, "Online Palmprint Identification," IEEE Trans. Pattern. Anal. Mach. Intell., vol. 25, no. 9, pp. 1041-1050, 2003. 
[9] A. Kumar and D. Zhang, "Integrating Shape and Texture for Hand Verification," in International Conference on Image and Graphics, Hong Kong, China, 2004, pp. 222-225.

[10] C. Poon, D. Wong, and H. Shen, "A New Method in Locating and Segmenting Palmprint into Region-of-Interest," in IEEE 17th Int. Conf. Pattern Recognition (ICPR'2004), vol. 4, Cambridge, UK, 2004, pp. 533-536.

[11] Y. Han, Z. Sun, F. Wang, and T. Tan, "Palmprint Recognition Under Unconstrained Scenes," in Asian Conference on Computer Vision, vol. LNCS 4844, Tokyo, Japan, 2007, pp. 1-11.

[12] W. Kong, D. Zhang, and W. Li, "Palmprint Feature Extraction Using 2-D Gabor Filters," Pattern Recognition, vol. 36, no. 10, pp. 2339-2347, 2003.

[13] D. Zhang, W. Zuo, and F. Yue, "A Comparative Study of Palmprint Recognition Algorithms," ACM Comput. Surv., vol. 44, no. 1, pp. 2:1$2: 37,2012$.

[14] J. You, W. Kong, D. Zhang, and K. Cheung, "On Hierarchical Palmprint Coding with Multiple Features for Personal Identification in Large Databases," IEEE Trans. Circuits and Systems for Video Technology, vol. 14, no. 2, pp. 234-243, 2004.

[15] Y. Wang and Q. Ruan, "A New Preprocessing Method of Palmprint (in Chinese)," Journal of Image and Graphics, vol. 13, no. 6, pp. 1115-1122, 2008 .

[16] M. Mu and Q. Ruan, "Region Covariance Matrices as Feature Descriptors for Palmprint Recognition Using Gabor Features," Int. J. Patt. Recog. Art. Intel., vol. 25, no. 4, pp. 513-528, 2011.

[17] S. Yanushkevich, D. Hurley, and P. Wang, "Pattern Recognition and Artificial Intelligence in Biometrics - Editorial," Int. J. Pattern Recogn. Artif. Intell., vol. 22, no. 3, pp. 367-369, 2008.

[18] P. Hiremath and C. Prabhakar, "Symbolic Factorial Discriminant Analysis for Illumination Invariant Face Recognition," Int. J. Pattern Recogn. Artif. Intell., vol. 22, no. 3, pp. 371-387, 2008.

[19] C. Queirolo, L. Silva, O. Bellon, and M. Segundo, "3D Face Recognition Using Simulated Annealing and the Surface Interpenetration Measure," 
IEEE Trans. Pattern Anal. Mach. Intell., vol. 32, no. 2, pp. $206-219$, 2010.

[20] J. Gu, J. Zhou, and C. Yang, "Fingerprint Recognition by Combining Global Structure and Local Cues," IEEE Trans. Image Process., vol. 15, no. 7, pp. 1952-1964, 2006.

[21] S. Srihari and H. Srinivasan, "Comparison of ROC and Likelihood Decision Methods in Automatic Fingerprint Verification," Int. J. Pattern Recogn. Artif. Intell., vol. 22, no. 3, pp. 535-553, 2008.

[22] X. Feng, X. Ding, Y. Wu, and P. Wang, "Classifier Combination and Its Application in Iris Recognition," Int. J. Pattern Recogn. Artif. Intell., vol. 22, no. 3, pp. 617-638, 2008.

[23] R. Sanchez-Reillo, A. Vazquez-Navarro, and J. Liu-Jimenez, "Biometric Identification Through Hand Geometry Measurements," IEEE Trans. Pattern Anal. Mach. Intell., vol. 22, no. 10, pp. 1168-1171, 2002.

[24] P. Smaragdis and M. Shashanka, "A Framework for Secure Speech Recognition," IEEE Trans. Audio Speech Lang. Process., vol. 15, no. 4, pp. 1404-1413, 2007.

[25] J. Yun, G. Abowd, J. Ryu, and W. Woo, "User Identification with User's Stepping Pattern Over the Ubifloor II," Int. J. Pattern Recogn. Artif. Intell., vol. 22, no. 3, pp. 497-514, 2008.

[26] H. Prakash and D. Guru, "Online Signature Verification and Recognition: An Approach Based on Symbolic Representation," IEEE Trans. Pattern Anal. Mach. Intell., vol. 31, no. 6, pp. 1059-1073, 2009.

[27] J. Alon, V. Athitsos, Q. Yuan, and S. Sclaroff, "A Unified Framework for Gesture Recognition and Spatiotemporal Gesture Segmentation," IEEE Trans. Pattern Anal. Mach. Intell., vol. 31, no. 9, pp. 1685-1699, 2009.

[28] C. Park, T. Choi, Y. Kim, S. Kim, J. Namkung, and J. Paik, "Multimodal Human Verification Using Face and Speech," in Proc. Fourth IEEE Int. Conf. Comput. Vis. Syst. (ICVS'06). New York, NY, USA: IEEE Computer Society, 2006, pp. 54.

[29] L. Hong and A. Jain, "Integrating Faces and Fingerprints for Personal Identification," IEEE Trans. Pattern Anal. Mach. Intell., vol. 20, no. 12, pp. 1295-1307, 1998. 
[30] J. Rokita, A. Krzyzak, and C. Suen, "Multimodal Biometrics by Face and Hand Images Taken by a Cell Phone Camera," Int. J. Pattern Recogn. Artif. Intell., vol. 22, no. 3, pp. 411-429, 2008.

[31] D. Zhang, Palmprint Authentication. Dordrecht: Kluwer Academic Publication, 2004.

[32] N. Duta, A. Jain, and K. Mardia, "Matching of Palmprint," Pattern Recognition Letters, vol. 23, no. 4, pp. 477-485, 2002.

[33] D. Zhang and W. Shu, "Two Novel Characteristics in Palmprint Verification: Datum Point Invariance and Line Feature Matching," Pattern Recognition Letters, vol. 32, no. 4, pp. 691-702, 1999.

[34] G. Lu, D. Zhang, and K. Wang, "Palmprint Recognition Using Eigenpalms Features," Pattern Recognit. Lett., vol. 24, no. 9-10, pp. 14631467, 2003.

[35] X. Wu, D. Zhang, and K. Wang, "Fisherpalms Based Palmprint Recognition," Pattern Recognit. Lett., vol. 24, no. 15, pp. 2829-2838, 2003.

[36] A. Kong, D. Zhang, and M. Kamel, "Palmprint Identification Using Feature-Level Fusion," Pattern Recognition, vol. 39, no. 3, pp. 478-487, 2006.

[37] A. Kong and D. Zhang, "Competitive Coding Scheme for Palmprint Verification," in Proc. 17th Int. Conf. Pattern Recognition, Cambridge, UK, 2004, pp. 520-523.

[38] X. Wu, K. Wang, and D. Zhang, "Palmprint Authentication Based on Orientation Code Matching," in: AVBPA 2005, Lecture Notes in Computer Science, vol. 3546, pp. 555-562, 2005.

[39] W. Jia, D. Huang, and D. Zhang, "Palmprint Verification Based on Robust Line Orientation Code," Pattern Recognition, vol. 41, no. 5, pp. 1521-1530, 2008.

[40] F. Yue, W. Zuo, D. Zhang, and K. Wang, "Orientation Selection Using Modified FCM for Competitive Code-based Palmprint Recognition," Pattern Recognition, vol. 42, no. 11, pp. 2841-2849, 2009.

[41] Z. Guo, D. Zhang, L. Zhang, and W. Zuo, "Palmprint Verification Using Binary Orientation Co-occurrence Vector," Pattern Recognit. Lett., vol. 30, no. 13, pp. 1219-1227, 2009. 
[42] X. Wu, K. Wang, and D. Zhang, "Palmprint Texture Analysis Using Derivative of Gaussian Filters," in Proc. IEEE Int. Conf. Computational Intelligence and Security, Guangzhou, China, 2006, pp. 751-754.

[43] W. Li, J. You, and D. Zhang, "Texture-based Palmprint Retrieval Using A Layered Search Scheme for Personal Identification," IEEE Trans. Multimedia, vol. 7, no. 5, pp. 891-898, 2005.

[44] X. Wu, K. Wang, and D. Zhang, "Wavelet Energy Feature Extraction and Matching for Palmprint Recognition," Journal of Computer Science and Technology, vol. 20, no. 5, pp. 411-418, 2005.

[45] J. Daugman, "Uncertainty Relation for Resolution in Space, Spatial Frequency, and Orientation Optimized by Two-dimensional Visual Cortical Filters," Optical Soc. Am., vol. 2, no. 7, pp. 1160-1169, 1985.

[46] T. Lee, "Image Representation Using 2D Gabor Wavelet," IEEE Trans. on Pattern Analysis and Machine Intelligence, vol. 18, no. 10, pp. 959971, 1996.

[47] M. Laadjel, A. Bouridane, F. Kurugollu, and S. Boussakta, "Palmprint Recognition Using Fisher-Gabor Feature Extraction," in Proc. IEEE International Conference on Acoustics, Speech and Signal Processing (ICASSP 2008), Las Vegas, Nevada, USA, 2008, pp. 1709-1712.

[48] X. Pan and Q. Ruan, "Palmprint Recognition Using Gabor Featurebased (2D) ${ }^{2}$ PCA," Neurocom. Lett., vol. 71, no. 13-15, pp. 3032-3036, 2008 .

[49] X. Pan and Q. Ruan, "Palmprint Recognition Using Gabor-based Local Invariant Features," Neurocom. Lett., vol. 72, no. 7-9, pp. 2040-2045, 2009.

[50] R. Chu, Z. Lei, Y. Han, R. He, and S. Li, "Learning Gabor Magnitude Features for Palmprint Recognition," in Proc. 8th Asian Conf. Computer Vision (ACCV2007), Tokyo, Japan, 2007, pp. 22-31.

[51] O. Tuzel, F. Porikli, and P. Meer, "Region Covariance: A Fast Descriptor for Detection and Classification," in Proc. 9th Eur. Conf. Comput. Vision (ECCV 2006), vol. LNCS 3952, Austria, 2006, pp. 589-600.

[52] O. Tuzel, F. Porikli, and P. Meer, "Human Detection via Classification on Riemannian Manifolds," in Proc. IEEE Conf. Comput. Vision Pattern Recog. (CVPR'07), Minneapolis, Minnesota, USA, 2007, pp. 1-8. 
[53] Y. Pang, Y. Yuan, and X. Li, "Gabor-based Region Covariance Matrices for Face Recognition," IEEE Trans. on Circuits and Systems for Video Technology, vol. 18, no. 7, pp. 989-993, 2008.

[54] J. Lu, Y. Zhao, and J. Hu, "Enhanced Gabor-based Region Covariance Matrices for Palmprint Recognition," Electron. Lett., vol. 45, no. 17, pp. 880-881, 2009.

[55] M. Mu and Q. Ruan, "Mean and Standard Deviation as Features for Palmprint Recognition Based on Gabor Filters," Int. J. Patt. Recog. Art. Intel., vol. 25, no. 4, pp. 491-512, 2011.

[56] D. Hu, G. Feng, and Z. Zhou, "Two-dimensional Locality Preserving Projections (2DLPP) with Its Application to Palmprint Recognition," Pattern Recognition, vol. 40, no. 1, pp. 339-342, 2007.

[57] S. Zhu, Y. Wu, and D. Mumford, "Minimax Entropy Principle and Its Applications to Texture Modeling," Neural Computation, vol. 9, no. 7, pp. 1627-1660, 1997.

[58] A. Kumar and D. Zhang, "Personal Authentication Using Multiple Palmprint Representation," Pattern Recognition, vol. 38, no. 10, pp. 1695-1704, 2005.

[59] W. Jia, B. Ling, K. Chau, and L. Heutte, "Palmprint Identification Using Restricted Fusion," Applied Mathematics and Computation, vol. 205, no. 2, pp. 916-926, 2008.

[60] S. Arivazhagan, L. Ganesan, and S. Priyal, "Texture Classification Using Gabor Wavelets Based Rotation Invariant Features," Pattern Recognition Letters, vol. 27, no. 16, pp. 1976-1982, 2006.

[61] J. Han and K. Ma, "Rotation-Invariant and Scale-Invariant Gabor Features for Texture Image Retrieval," Image and Vision Computing, vol. 25, no. 9, pp. 1474-1481, 2007.

[62] J. Beck, A. Sutter, and R. Ivry, "Spatial Frequency Channels and Perceptual Grouping in Texture Segmentation," Computer Vision, Graphics, Image Processing, vol. 37, no. 2, pp. 299-325, 1987.

[63] M. Mu, Q. Ruan, and S. Guo, "Shift and Gray Scale Invariant Features for Palmprint Recognition Using Complex Directional Wavelet and Local Binary Pattern," Neurocomputing, vol. 74, no. 17, pp. 3351-3360, 2011.

[64] S. Pankanti, R. Bolle, and A. Jain, "Biometrics: The Future of Identification," IEEE Comput., vol. 33, no. 2, pp. 46-49, 2000. 
[65] D. Huang, W. Jia, and D. Zhang, "Palmprint Verification Based on Principal Lines," Pattern Recognition, vol. 41, no. 4, pp. 1316-1328, 2008.

[66] Z. Zhao, D. Huang, and W. Jia, "Palmprint Recognition with 2DPCA+PCA Based on Modular Neural Networks," Neurocomputing Letters, vol. 71, no. 1-3, pp. 448-454, 2007.

[67] M. Ekinci and M. Aykut, "Palmprint Recognition by Applying Waveletased Kernel PCA," Journal of Computer Science and Technology, vol. 23, no. 5, pp. 851-861, 2008.

[68] Y. Wang, Q. Ruan, and X. Pan, "Palmprint Recognition Method Using Dual-Tree Complex Wavelet Transform and Local Binary Pattern Histogram," in Proc. Int. Symp. Intell. Signal Process. Commun. Syst. (ISPACS'07), Xiamen, China, 2007, pp. 646-649.

[69] T. Ojala, M. Pietikäinen, and T. Mäenpää, "Multiresolution Gray-cale and Rotaion Invariant Texture Classification with Local Binary Patterns," IEEE Trans. Pattern Anal. Mach. Intell., vol. 24, no. 7, pp. 971-987, 2002.

[70] W. Zhang, S. Shan, W. Gao, and H. Zhang, "Local Gabor Binary Pattern Histogram Sequence (LGBPHS): A Novel Non-Statistical Model for Face Representation and Recognition," in Proc. 10th Int. Conf. Computer Vision, vol. 1, Beijing, China, 2005, pp. 786-791.

[71] Z. Lei, S. Liao, R. He, M. Pietikänen, and S. Li, "Gabor Volume Based Local Binary Pattern for Face Representation and Recognition," in Proc. IEEE Int. Conf. Automatic Face \& Gesture Recognition, Amsterdam, The Netherlands, 2008, pp. 1-6.

[72] B. Zhang, Z. Wang, and B. Zhong, "Kernel Learning of Histogram of Local Gabor Phase Patterns for Face Recognition," Eurasip Journal on Advances in Signal Processing, vol. 2008, pp. 1-9, 2008.

[73] X. Tan and B. Triggs, "Enhanced Local Texture Feature Sets for Face Recognition Under Difficult Lighting Conditions," IEEE Trans. image process., vol. 19, no. 6, pp. 168-182, 2010.

[74] S. Liao, W. Law, and C. Chung, "Dominant Local Binary Patterns for Texture Classification," IEEE Trans. image process., vol. 18, no. 5, pp. 1107-1118, 2009. 
[75] M. Pietikäinen and C. Schmid, "Description of Interest Regions with Local Binary Patterns," Pattern Recognition, vol. 42, no. 3, pp. 425436, 2009.

[76] Z. Guo, L. Zhang, and D. Zhang, "Rotation Invariant Texture Classification Using LBP Variance (LBPV) with Global Matching," Pattern Recognition, vol. 43, no. 3, pp. 706-719, 2010.

[77] B. Zhang, Y. Gao, S. Zhao, and J. Liu, "Local Derivative Pattern Versus Local Binary Pattern: Face Recognition with High-order Local Pattern Descriptor," IEEE Trans. Image Process., vol. 19, no. 2, pp. 533-544, 2010.

[78] Z. Guo, L. Zhang, and D. Zhang, "A Completed Modeling of Local Binary Pattern Operator for Texture Classification," IEEE Trans. image process., vol. 19, no. 6, pp. 1657-1663, 2010.

[79] W. Zhang, S. Shan, X. Chen, and W. Gao, "Are Gabor Phases Really Useless for Face Recognition?" Pattern Analysis \& Applications, vol. 12, no. 3, pp. 301-307, 2009.

[80] B. Zhang, S. Shan, X. Chen, and W. Gao, "Histogram of Gabor Phase Patterns (HGPP): A Novel Object Representation Approach for Face Recognition," IEEE Trans. image process., vol. 16, no. 1, pp. 57-68, 2007.

[81] S. Xie, S. Shan, X. Chen, and J. Chen, "Fusing Local Patterns of Gabor Magnitude and Phase for Face Recognition," IEEE Trans. Image Process., vol. 19, no. 5, pp. 1349-1361, 2010.

[82] T. Nguyen and S. Oraintara, "The Shiftable Complex Directional Pyramid - Part I: Theoretical Aspects," IEEE Trans. Signal Process., vol. 56, no. $10-1$, pp. 4651-4660, 2008.

[83] T. Nguyen and S. Oraintara, "The Shiftable Complex Directional Pyramid - part II: Implementation and Applications," IEEE Trans. Signal Process., vol. 56, no. 10-1, pp. 4661-4672, 2008.

[84] E. Simoncelli, W. Freeman, E. Adelson, and D. Heeger, "Shiftable Multiscale Transform," IEEE Trans. Inf. Theory, vol. 38, no. 2, pp. 587-607, 1992.

[85] M. Do and M. Vetterli, "The Contourlet Transform: An Efficient Directional Multiresolution Image Representation," IEEE Trans. Image Processing, vol. 14, no. 12, pp. 2091-2106, 2005. 
[86] I. Selesnick, R. Baraniuk, and N. Kingsbury, "The Dual-Tree Complex Wavelet Transform," IEEE Signal Processing Magazine, vol. 22, no. 6, pp. 123-151, 2005.

[87] A. Cunha, J. Zhou, and M. Do, "The Nonsubsampled Contourlet Transform: Theory, Design, and Applications," IEEE Trans. Image Process., vol. 15, no. 10, pp. 3089-3101, 2006.

[88] M. Mu, Q. Ruan, L. Spreeuwers, and R. Veldhuis, "Fourier Spectral of Palmcode as Descriptor for Palmprint Recognition," in Int. Conf. Pattern Recognition Applications and Methods (ICPRAM), Barcelona, Spain, 2013.

[89] W. Shu and D. Zhang, "Palmprint Verification: An Implementation of Biometric Technology," in Proc. 14th Int. Conf. Pattern Recogn., Brisbane, Australia, 1998, pp. 219-221.

[90] W. Shu and D. Zhang, "Automated Personal Identification by Palmprint," Optical Engineering, vol. 37, no. 8, pp. 2659-2362, 1998.

[91] R. Rowe, U. Uludag, M. Demirkus, S. Parthasaradhi, and A. Jain, "A Multispectral Whole-hand Biometric Authentication System," in Proc. Biometrics Symposium, Baltimore, MD, 2007, pp. 1-6.

[92] Z. Guo, L. Zhang, and D. Zhang, "Feature Band Selection for Multispectral Palmprint Recognition," in Proc. 20th Int. Conf. Pattern Recognition, Istanbul, Turkey, 2010, pp. 1136-1139.

[93] A. Kong, D. Zhang, and M. Kamel, "Palmprint Identication Using Feature-level Fusion,", Pattern Recognition, vol. 39, no. 3, pp. 478-487, 2006.

[94] Z. Sun, T. Tan, Y. Wang, and S. Li, "Ordinal Palmprint Representation for Personal Identification," in Proc. IEEE Int. Conf. Computer Vision and Pattern Recognition, San Diego, CA, USA, 2005, pp. 279-284.

[95] W. Zuo, Z. Lin, Z. Guo, and D. Zhang, "The Multiscale Competitive Code via Sparse Representation for Palmprint Verification," in Proc. 23rd IEEE Int. Conf. Computer Vision and Pattern Recognition (CVPR), San Francisco, CA, USA, 2010, pp. 2265-2272.

[96] W. Jia, D. Huang, D. Tao, and D. Zhang, "Palmprint Identification Based on Directional Representation," in IEEE Int. Conf. Systems, Man and Cybernetics 2008 (SMC2008), Singapore, 2008, pp. 1562-1567. 
[97] F. Yue, W. Zuo, D. Zhang, and B. Li, "Fast Palmprint Identification with Multiple Templates per Subject," Pattern Recognition Letters, vol. 32, no. 8, pp. 1108-1118, 2011.

[98] F. Yue, W. Zuo, K. Wang, and D. Zhang, "A Performance Evaluation of Filter Design and Coding Schemes for Palmprint Recognition," in Proc. 19th Int. Conf. Pattern Recognition (ICPR'08), Tampa, Florida, USA, 2008, pp. 1-4.

[99] J. Daugman, "High Confidence Visual Recognition of Persons by A Test of Statistical Independence," IEEE Trans. Pattern Anal. Mach. Intell., vol. 15, no. 11, pp. 1148-1161, 1993.

[100] D. Zhang, X. You, P. Wang, S. Yanushkevich, and Y. Tang, "Facial Biometrics Using Nontensor Product Wavelet and 2D Discriminant Techniques," Int. J. Patt. Recog. Art. Intel., vol. 23, no. 3, pp. 521-543, 2009 .

[101] J. Yang and C. Liu, "Horizontal and Vertical 2DPCA-based Discriminant Analysis for Face Verification on A Large-scale Database," IEEE Transactions on Information Forensics and Security, vol. 2, no. 4, pp. 781-792, 2007.

[102] M. Mu, Q. Ruan, X. Shao, L.J. Spreeuwers, and R.N.J. Veldhuis, "Binary Gabor Statistical Features for Palmprint Template Protection," in Joint IAPR International Workshop, SSPR\&SPR 2012, vol. LNCS 7626. Hiroshima, Japan: Springer Berlin Heidelberg, 2012, pp. 593-601.

[103] R. Richard, Theory and practice of error control codes. Addison-Wesley Publishing Company, Inc., 1983.

[104] X. Shao, H. Xu, R.N.J. Veldhuis, and C.H. Slump, "A Concatenated Coding Scheme for Biometric Template Protection," in Proc. IEEE Int. Conf. Acoustics, Speech and Signal Processing (ICASSP), Japan, 2012.

[105] M. Mu, Q. Ruan, X. Shao, L.J. Spreeuwers, and R.N.J. Veldhuis, "Bits Extraction for Palmprint Template Protection with Gabor Magnitude and Multi-bit Quantization," in the 6th IAPR international conference on Biometrics (ICB-2013), Madrid, Spain, 2013.

[106] A. Jain and K. Nandakumar, "Biometric Authentication: System Security and User Privacy," IEEE Trans. Comput., vol. 45, no. 11, pp. 87-92, 2012 . 
[107] P. Tuyls, A. Akkermans, T. Kevenaar, G. Schrijen, A. Bazen, and R.N.J. Veldhuis, "Practical Biometric Authentication with Template Protection," in the 5th International Confernce on Audio- and VideoBased Biometric Person Authentication, vol. 3546, NY, USA, 2005.

[108] F. Hao, R. Anderson, and J. Daugman, "Combining Cryptography with Biometrics Effectively," IEEE Trans. Comput., vol. 55, no. 9, pp. 10811088, 2006.

[109] C. Chen, R.N.J. Veldhuis, T.A. Kevenaar, and T.H. Akkermans, "Biometric Quantization Through Detection Rate Optimized Bit Allocation," EURASIP Journal on Advances in Signal Processing, vol. 2009, pp. 1-16, 2009. 


\section{Acknowledgements}

Five years ago I was recommended to be a $\mathrm{PhD}$ student in Institute of Information Science, Beijing Jiaotong University, China, where I started my PhD research journey. In the year 2011, I came to The Netherlands to continue the research work at the chair of Signals and Systems (SAS) of the University of Twente. In retrospect, it has been an amazing experience. I would have never pictured my $\mathrm{PhD}$ life the way it is now when it started. Here I would like to express my gratitude to those people who have contributed to the research findings, the thesis progress and my magic $\mathrm{PhD}$ journey.

First of all, I am thinking particularly of my supervisor, Raymond Veldhuis. I respect him very much for his profound professional skills, his enthusiasm for supervision, and his cheerful attitude to life. I was someone when I came to Holland, and now I take pride in keeping growing into a better someone under his guidance and encouragement. Thanks to our discussions of research, life, and human being, I got the purpose and meaning to my lives (why I am here) in the Netherlands, which has made my research work more enjoyable and life adventure more pleasurable. And then, I am grateful to my second supervisor Luuk Spreeuwers, and my collaborator Xiaoying Shao. They have stood by me during the research progress and helped to shape the thesis by their comments and suggestions at various stages. Invaluable comments were also obtained from my examiners. I would also like to thank them for taking time to read my thesis carefully.

I would like to thank my colleagues from the Signals and Systems (SAS) group for their friendship. My thanks first goes to Sandra and Geert Jan. Thank you for being there whenever I was in trouble. And Geert Jan, especially thanks for the "Cheer up and Smile" picture you sent to me on that day I was 
badly sad. I would like you to know how precious it was for me. Xiaoying, thanks for your support in my being in our group and living in Enschede! Rita, Bianca, Julia, Sjoerd and Gert, it is a great pleasure we are officemates. I like our tea and soup time especially. Thanks for sharing and caring! And I am much indebted to Tauseef, Abhishek, Cris, Robin, Pina, and Yuxi for sharing research experience and ideas. They were very inspiring and supportive occasions when Tauseef explained to me what the forensic science is about, and when Abhishek described to me how the Bayes network works. Particularly, I would like to thank Tauseef, Renxuan, Abhishek, Pina and Chanjuan for their emotional support during those suffering moments in some rainy days. Also, I would like to thank Peng Jin, Tijmen, Bram, and Jasper for those happy time we spent together. Especially, many thanks to Tijmen for his support on our cooperative work as the assistant teachers. Further, I would like to thank Ruifang, Ram, and Anna. Even though we had less time being together in SAS group, those discussions on research and funny talks about life with you all have influenced me in many positive ways. And finally, I would like to say thanks to all my colleagues for creating such a cheerful working atmosphere.

I would like to thank my Chinese friends living in Enschede and Delft for their friendship. Thanks for sharing my happiness and sadness. The joyful moments we had together during travelling and dinner colors my happy memories of $\mathrm{PhD}$ journey.

I would also like to thank my colleagues and classmates in Institute of Information Science, Beijing Jiaotong University, China, where I started my PhD. More than anyone else, my thanks go to Professor Qiuqi Ruan, who has inspired me to be a researcher and given me great support in my first several $\mathrm{PhD}$ years. I am honored to be one of his students. His sincere attitude on research impressed me deeply. Thanks, my dear teacher!

Finally, many thanks go to my family in China. Thanks for supporting me throughout my endeavors.

This research was part-funded by Institute of Information Science, Beijing Jiaotong University, China, part-funded by Chinese Scholarship Council (CSC), and part-funded by the lab of Signals and Systems group, University of Twente, The Netherlands. 


\section{Curriculum vitae}

Meiru Mu was born in Shanxi, China, on October 27, 1984. She received the B.E. degree in Electronic and Information Engineering from Communication University of China, Beijing, China, in 2007. In September 2007, she was accepted as a Master student by the School of Computer and Information Technology, Beijing Jiaotong University, China. One year later, she was recommended to be a $\mathrm{PhD}$ candidate at Institute of Information Science, Beijing Jiaotong Uni-

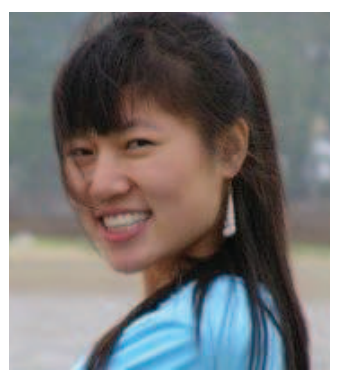
versity, under the supervision of Prof. Qiuqi Ruan. The research topic was mainly about palmprint recognition technology. The main efforts have been put on the study of feature extraction and matching algorithms for online low-resolution palmprint recognition.

From September 2011, she started to do research work at the Signals and Systems group, University of Twente, The Netherlands, as a $\mathrm{PhD}$ student supported by the Scholarship from Chinese Scholarship Council (CSC), under the supervision of Raymond Veldhuis and assistance of Luuk Spreeuwers and Xiaoying Shao. The research topic focused on the study of binary feature extraction algorithms for the improvement of palmprint template protection technology. In April 2013, she finished her Ph.D. thesis entitled by "texture representation for low-resolution palmprint recognition". Since May 2013, she has been employed as post-doc at the Signals and Systems group, University of Twente, The Netherlands. Her current work is about face recognition for the mobile law enforcement cameras. 


\section{List of publications}

- M. Mu, Q. Ruan and Y. Shen, "Palmprint Recognition Based on Discriminative Local Binary Patterns Statistic Feature" in Proc. 2010 Int. Conf. Signal Acquisition and Processing (ICSAP'10), Bangalore, India, pp. 193197, 2010.

- M. Mu and Q. Ruan, "Palmprint Recognition Based on Statistical Local Binary Orientation Code," Journal of Electronic Science and Technology of China, vol. 8, no. 3, pp. 230-236, 2010.

- M. Mu, Q. Ruan and Y. Ming, "Shape Parameters of Gaussian as Descriptor for Palmprint Recognition Based on Dual-tree Complex Wavelet Transform" in Proc. 2010 IEEE 10th Int. Conf. Signal Processing (ICSP), Beijing, China, pp. 1406-1409, 2010.

- M. Mu and Q. Ruan, "Mean and Standard Deviation as Features for Palmprint Recognition Based on Gabor Filters," Int. J. Patt. Recog. Art. Intel., vol. 25, no. 4, pp. 491-512, 2011.

- M. Mu and Q. Ruan, "Region Covariance Matrices as Feature Descriptors for Palmprint Recognition Using Gabor Features," Int. J. Patt. Recog. Art. Intel., vol. 25, no. 4, pp. 513-528, 2011.

- M. Mu, Q. Ruan and S. Guo, "Shift and Gray Scale Invariant Features for Palmprint Recognition Using Complex Directional Wavelet and Local Binary Pattern," Neurocomputing, vol. 74, no. 17, pp. 3351-3360, 2011.

- M. Mu, Q. Ruan, X. Shao, L.J. Spreeuwers and R.N.J. Veldhuis, "Binary Palmprint Representation for Feature Template Protection," in Proc. 33rd WIC Symposium on Information Theory in the Benelux, Boekelo, the Netherlands, pp. 118-125, 2012.

- M. Mu, Q. Ruan, X. Shao, L.J. Spreeuwers and R.N.J. Veldhuis, "Binary Gabor Statistical Features for Palmprint Template Protection," in Proc. Joint IAPR International Workshop, SSPR\&SPR 2012, Hiroshima, Japan, LNCS 7626, pp. 593-601, 2012.

- M. Mu, Q. Ruan, L.J. Spreeuwers and R.N.J. Veldhuis, "Fourier Spectral of PalmCode as Descriptor for Palmprint Recognition," in Proc. Int. Conf. Pattern Recognition Applications and Methods (ICPRAM), Barcelona, Spain, 2013.

- M. Mu, Q. Ruan, X. Shao, L.J. Spreeuwers and R.N.J. Veldhuis, "Bits Extraction for Palmprint Template Protection with Gabor Magnitude and Multi-bit Quantization," in Proc. the 6th IAPR international conference on Biometrics (ICB-2013), Madrid, Spain, 2013. 